\title{
Laplace Approximations for Censored Linear Regression Models
}

by

GEORGE PAPANDONATOS and SEYMOUR GEISSER

SUNY at Buffalo and University of Minnesota

Technical Report No. 603

June 1995 


\title{
Laplace Approximations for Censored Linear Regression Models ${ }^{1}$ \\ GEORGE D. PAPANDONATOS \\ Department of Statistics \\ 242 Farber Hall, 3435 Main St. \\ School of Medicine and Biomedical Sciences \\ Buffalo, N.Y. 14214-300 \\ and \\ SEYMOUR GEISSER \\ School of Statistics, University of Minnesota \\ 270 Vincent Hall, 206 Church St. SE \\ Minneapolis, MN 55455 \\ 2 June, 1995
}

\begin{abstract}
We consider approximate Bayesian inference about scalar parameters of linear regression models with possible censoring. A second order expansion of their Laplace posterior is seen to have a simple and intuitive form for logconcave error densities with nondecreasing hazard functicns. The accuracy of the approximations is assessed for Normal and Gumbel errors, when the number of regressors increases with sample size. Perturbations of the prior and the likelihood are seen to be easily accomodated within our framework. Links with the work of DiCiccio et al. (1990) and Viveros \& Sprott (1987) extend the applicability of our results to conditional frequentist inference based on likelihood ratio statistics.
\end{abstract}

Key words. Bayesian inference; Censored data; Generalised Gamma Distribution; Laplace Approximation; Linear Regression; Location-Scale Model; Second Order Asymptotics.

\footnotetext{
${ }^{1}$ Research partially supported by National Institutes of General Medical Sciences Grant GM-25271.
} 


\section{Introduction.}

In linear regression models with known error distributions and possible censoring, the Bayesian posterior distributions of parametric functions of interest are usually unavailable in closed form and need to be evaluated numerically. For multidimensional problems, numerical integration can be too cumbersome and time-consuming and analysts often resort to simulation and/or analytic approximation. In this paper, we restrict our attention to second order expansions of univariate posterior densities, under arbitrary priors and noninformative censoring patterns. We then show how these simplify for individual regression coefficients, the scale parameter, arbitrary quantiles of the response and realised errors from the model. We adopt data-dependent reparameterisations that extend the applicability of our results to the approximation of the conditional frequentist distribution of pivotal quantities given a set of ancillaries, when such statistics can be found. Our approach is similar to DiCiccio et al. (1990) and DiCiccio \& Field (1991), in that we first expand and then integrate asymptotically to second order the univariate Laplace posterior of individual parameters rather than their exact joint posterior, thus trading a hard integration problem for a simpler differentiation one. By restricting our attention to linear regression models, we obtain simplifications of fifth and sixth order sums of derivative arrays that eluded the aforementioned authors. Also, by expressing our results in terms of the first four derivatives of the Laplace posterior, evaluated at the MLE, we extend to regression models the recommendations of Viveros \& Sprott (1987), for the fitting of parametric families of densities by derivative matching. We show that the results they obtained for the location and scale parameters also apply to quantiles of the response and need not be restricted to location-scale models. We examine the finite sample performance of our approximations, within the context of the log generalised Gamma family of distributions, and show that, where comparable, our results can be viewed as modifications of the expansions in Johnson \& Kotz (1970), that separate the effect increasing the degrees of freedom into a part due to the sample size an one due to increasing the dimensionality of the model. This distinction allows us to assess the sensitivity of our approximations to increasing the number of parameters and the degrees of freedom simultaneously. We conclude with an example which is suggestive of the ease with which $O(1)$ perturbations of the likelihood can be accommodated within our framework.

\section{Expansions based on Laplace posteriors}

Consider a continuous random variable $V_{n} \sim O_{p}(1)$ as some index $n$ grows indefinitely. Suppressing the dependence on $n$, assume that the exact density $f_{V}(v)$ has a unique maximum at 0 and satisfies $\log f_{V}(v) \approx \log \tilde{f}_{V}(v)$ for $v \sim O(1)$, where we define

$$
\tilde{f}_{V}(v)=c(2 \pi)^{-\frac{1}{2}} \exp \left[d_{1} v-\frac{1}{2}\left(1-d_{2}\right) v^{2}+\frac{1}{6} F_{3} v^{3}+\frac{1}{24} F_{4} v^{4}\right]
$$


for some $d_{1}, F_{3} \sim O\left(n^{-\frac{1}{2}}\right), d_{2}, F_{4} \sim O\left(n^{-1}\right) .^{2}$ The constant of proportionality is chosen so that $\int \tilde{f}_{V}(v) d v \approx 1$, e.g. by letting $c \approx \exp \left[-\frac{1}{24}\left(12 d_{1}^{2}+12 d_{1} F_{3}+5 F_{3}^{2}+3 F_{4}+12 d_{2}\right)\right]$.

Assuming it exists, the cumulant generating function of $V$ can be approximated by

$$
K_{V}(t) \approx\left(d_{1}+\frac{1}{2} F_{3}\right) t+\frac{1}{2 !}\left(1+d_{1} F_{3}+F_{3}^{2}+\frac{1}{2} F_{4}+d_{2}\right) t^{2}+\frac{1}{3 !} F_{3} t^{3}+\frac{1}{4 !}\left(F_{4}+3 F_{3}^{2}\right) t^{4}
$$

It follows that the mean, standard deviation, skewness and kurtosis of $V$ satisfy

$$
\mu \approx\left(d_{1}+\frac{1}{2} F_{3}\right), \sigma \approx 1+\frac{1}{2}\left(d_{1} F_{3}+F_{3}^{2}+\frac{1}{2} F_{4}+d_{2}\right), \gamma_{3} \approx F_{3}, \gamma_{4} \approx F_{4}+3 F_{3}^{2}
$$

respectively. Furthermore, the distribution function of $V$ can be approximated as

$$
\begin{aligned}
F_{V}(v) & \approx \Phi(v)-\phi(v) p(v) \\
& \approx \Phi(v+\epsilon(v)) \\
& \approx \Phi\left((r(v)-a)\left(1+b-a^{2}\right)^{-\frac{1}{2}}\right)
\end{aligned}
$$

where

$$
\begin{aligned}
p(v)= & \frac{1}{3}\left(3 d_{1}+F_{3}\right)+\frac{1}{24}\left(12 d_{1}^{2}+12 d_{1} F_{3}+5 F_{3}^{2}+3 F_{4}+12 d_{2}\right) v+ \\
& \frac{1}{6} F_{3} v^{2}+\frac{1}{72}\left(12 d_{1} F_{3}+5 F_{3}^{2}+3 F_{4}\right) v^{3}+\frac{1}{72} F_{3}^{2} v^{5} \\
-\epsilon(v)= & \frac{1}{3}\left(3 d_{1}+F_{3}\right)+\frac{1}{72}\left(12 d_{1} F_{3}+11 F_{3}^{2}+9 F_{4}+36 d_{2}\right) v+ \\
& \frac{1}{6} F_{3} v^{2}+\frac{1}{72}\left(3 F_{4}+F_{3}^{2}\right) v^{3} \\
r(v)= & v-\frac{1}{6} F_{3} v^{2}-\frac{1}{72}\left(3 F_{4}+F_{3}^{2}\right) v^{3} \\
a= & \frac{1}{3}\left(3 d_{1}+F_{3}\right), \\
b= & \left(d_{1}^{2}+d_{1} F_{3}+\frac{5}{12} F_{3}^{2}+\frac{1}{4} F_{4}+d_{2}\right)
\end{aligned}
$$

All three approximations to $F_{V}(v)$ are useful. Expression (2.6) suggests $r(v)$ as an obvious normalising tranformation of $V$, its significance to be explored later on. Expression (2.4) shows that the probability content of intervals symmetric around zero approaches its normal limit faster than that of individual tails, since $F_{V}(v)-F_{V}(-v) \approx 2 \Phi(v)-1-\phi(v) q(v)$, where

$q(v)=\frac{1}{12}\left(12 d_{1}^{2}+12 d_{1} F_{3}+5 F_{3}^{2}+3 F_{4}+12 d_{2}\right) v+\frac{1}{36}\left(12 d_{1} F_{3}+5 F_{3}^{2}+3 F_{4}\right) v^{3}+\frac{1}{36} F_{3}^{2} v^{5}$

\footnotetext{
${ }^{2}$ Approximate equality up to but not including $O\left(n^{-\frac{s}{2}}\right)$ terms will be denoted by the $\approx$ symbol.
} 


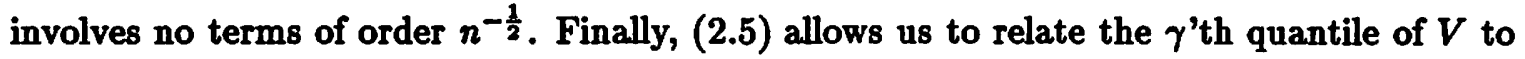
the $\gamma^{\prime}$ th normal quantile $z_{\gamma}$ via

$v_{\gamma} \approx z_{\gamma}+d_{1}+\frac{1}{3} F_{3}+\left(\frac{1}{2} d_{1} F_{3}+\frac{19}{72} F_{3}^{2}+\frac{1}{8} F_{4}+\frac{1}{2} d_{2}\right) z_{\gamma}+\frac{1}{6} F_{3} z_{\gamma}^{2}+\frac{1}{72}\left(5 F_{3}^{2}+3 F_{4}\right) z_{\gamma}^{3}$

An equivalent approximation to $v_{\gamma}$ can be obtained from (2.6). It is given by

$$
v_{\gamma} \approx r_{\gamma}+\frac{1}{6} F_{3} r_{\gamma}^{2}+\frac{1}{72}\left(5 F_{3}^{2}+3 F_{4}\right) r_{\gamma}^{3}
$$

with $r_{\gamma}=a+z_{\gamma}\left(1+b-a^{2}\right)^{\frac{1}{2}}$ and often performs better in small samples.

If one is willing to hazard a guess at the tail behaviour of the exact density of $V$, departures from normality due to skewness and kurtosis can also be accommodated by matching the derivatives of $\log \tilde{f}_{V}(v)$ at zero to those of a target density from a appropriate parametric family of distributions.

One possible strategy is to first set $\tilde{F}_{3}=F_{3}\left(1-d_{2}\right)^{-\frac{3}{2}}, \tilde{F}_{4}=F_{4}\left(1-d_{2}\right)^{-2}$ and then if $\tilde{F}_{3}=\tilde{F}_{4}=0$, treat $V$ as a $N\left(\frac{d 1}{1-d_{2}}, \frac{1}{1-d_{2}}\right)$ variable, else, if $\tilde{F}_{3}=d_{1}=0$ and $0<\tilde{F}_{4}<6$, assume a $t_{\nu}\left(0, \frac{\nu+1}{\nu} \frac{1}{1-d_{2}}\right)$ distribution for $V$, with $\nu=6 / \tilde{F}_{4}-1$ degrees of freedom,

else, if $d_{1}, \tilde{F}_{3}, \tilde{F}_{4} \neq 0$ and $h=\sqrt{3 \tilde{F}_{3}^{2}+2 \tilde{F}_{4}}>0$ is well defined, assume that $\left(V-\mu_{0}\right) / \sigma_{0}$ is distributed as the logarithm of an $F_{m_{0}, n_{0}}$ variable where the parameters $\mu_{0}, \sigma_{0}, m_{0}, n_{0}$ are determined by :

$$
\begin{array}{rlrl}
n_{1}^{-1} & \equiv \frac{1}{4} h\left(h+\tilde{F}_{3}\right), & m_{1}^{-1} \equiv \frac{1}{4} h\left(h-\tilde{F}_{3}\right), & \sigma_{0}^{-2} \equiv 2\left(n_{1}^{-1}+m_{1}^{-1}\right)\left(1-d_{2}\right), \\
n_{0} & \equiv n_{1}-2 \sigma_{0} d_{1}, \quad m_{0} \equiv m_{1}+2 \sigma_{0} d_{1}, \quad \mu_{0} \equiv \sigma_{0}\left[\log \left(\frac{m_{0}}{m_{1}}\right)-\log \left(\frac{n_{0}}{n_{1}}\right)\right] .
\end{array}
$$

Moments, quantiles and percentiles of the above distributions are readily available from standard computer packages and provide an alternative to the Edgeworth-type expansions described earlier, closer in spirit to the fitting of Pearson densities. It should be noted that matching derivatives at the mode of $\log \tilde{f}_{V}(v)$ itself, might provide a better fit, but in general $\tilde{v}$ is not available and using it will not lead to as many simplifications.

Up to now we have not explained how the density expansion in (2.1) may have arisen. To this effect, consider a continuous random vector $\omega \in \Re^{p}$ whose density is given by $f(\omega) \propto \pi(\omega) \exp L(\omega)$ where $L$ and its partial derivatives are $O(n)$, as some index $n$ grows without bound, and $\pi \sim O(1)$ is a strictly positive smooth function, such that $f$ is integrable for $n$ large enough. It may help to think of $f$ as the posterior of a parameter vector $\omega$ with $\pi$ as its - possibly improper - prior distribution and $L$ the $\log$-likelihood based on a sample of $n$ observations. This need not be the case in actual applications, where the prior could be absorbed in the exponent and $\pi$ taken as a constant. Alternatively, $\pi$ could be the ratio of the new to the original posterior in a Bayesian robustness analysis or the inverse of the 
likelihood contribution of an individual case in an influence analysis based on single case deletion.

Assume that interest centers on a single coordinates of $\omega$. Without loss of generality, we take it to be the first and partition $\omega$ accordingly into $\left(\omega_{1}, \omega_{2}^{T}\right)$. Suppressing the dependence on $n$, we let $\hat{\omega}$ to be the maximiser of $L$ and assume that $(\omega-\hat{\omega}) \sim O_{p}\left(n^{-\frac{1}{2}}\right)$. An approximation to the marginal density of $\omega_{1}$, based on the Laplace method for asymptotic evaluation of integrals, has been suggested by Leonard (1982) and Tierney \& Kadane (1986). It is based on large sample considerations and is sensitive to all but mild multimodality, so we would expect it to be affected by parameterisation and perform best where a normal approximation does well itself. Unlike the normal approximation though, it is flexible enough to accommodate both skewness and kurtosis. It was originally given in terms of the constrained mode of $f$ for $\omega_{1}$ fixed. Later, Kass et. al. (1990) showed that it could also be based on the constrained mode $\hat{\omega}\left(\omega_{1}\right)$ of $L$. This latter form is more suited to our purposes, since we may want to obtain results under a range of priors and drop/add individual cases.

Our choice of the Laplace approximation $\tilde{f}$ to the marginal posterior density of $\omega_{1}$ is then given by:

$$
\tilde{f}\left(\omega_{1}\right)=c(2 \pi)^{-\frac{1}{2}} \exp \left[w\left(\omega_{1}\right)+R\left(\omega_{1}\right)-\frac{1}{2} h\left(\omega_{1}\right)\right]
$$

where

$$
\begin{aligned}
& w\left(\omega_{1}\right)=\log \pi\left(\hat{\omega}\left(\omega_{1}\right)\right)-\log \pi(\hat{\omega}), \\
& h\left(\omega_{1}\right)=\log \left|\left(\frac{\partial^{2} L(\hat{\omega})}{\partial \omega_{2} \partial \omega_{2}^{T}}\right)^{-1}\left(\frac{\partial^{2} L\left(\hat{\omega}\left(\omega_{1}\right)\right)}{\partial \omega_{2} \partial \omega_{2}^{T}}\right)\right|, \\
& R\left(\omega_{1}\right)=L\left(\hat{\omega}\left(\omega_{1}\right)\right)-L(\hat{\omega}),
\end{aligned}
$$

and $c$ is a normalising constant that insures $\tilde{f}$ integrates to $1+O\left(n^{-\frac{3}{2}}\right)$. Kass et al. (1990) show that the error in the Laplace approximation can be expressed as $f\left(\omega_{1}\right)=$ $\tilde{f}\left(\omega_{1}\right)\left[1+O\left(n^{-1}\right)\right]$ uniformly in $\omega_{1}$, for $\left(\omega_{1}-\hat{\omega}_{1}\right) \sim O(1)$. An argument presented in Tierney $\&$ Kadane (1986, sec. 4) then shows that, if attention is restricted to $\left(\omega_{1}-\hat{\omega}_{1}\right) \sim O\left(n^{-\frac{1}{2}}\right)$, the normalised Laplace approximation satisfies $f\left(\omega_{1}\right)=\tilde{f}\left(\omega_{1}\right)\left[1+O\left(n^{-\frac{3}{2}}\right)\right]$ or, equivalently, $\log f\left(\omega_{1}\right)=\log \tilde{f}\left(\omega_{1}\right)+O\left(n^{-\frac{3}{2}}\right)$. This is to be compared with the usual normal approximation, whose absolute error is $O\left(n^{-\frac{1}{2}}\right)$ in $O\left(n^{-\frac{1}{2}}\right)$ neighbourhoods of the mode. The setup described above closely resembles the one described by DiCiccio et al. $(1990,1991)$ and is not entirely satisfactory since it omits any reference to the probabilistic mechanism that generates the data. In practice, extra conditions will have to be imposed to ensure that well-behaved sequences of data occur with probability one. Detailed regularity conditions and error bounds are given in Kass et al. (1990), whose approach is similar to Chen (1985) in separating the deterministic and probabilistic parts of the problem. The conditions they provide are closely related to the ones ensuring consistency and asymptotic normality of 
the MLE, so that in practice we expect our results to be valid under smooth, relatively flat priors for models where the MLE is asymptotically normal.

The above error bounds also imply that the Laplace approximation to the marginal density of $\omega_{1}$ has the same relative error as its Edgeworth-type expansion that includes two terms beyond the normal, i.e. we incur no further error by expanding $w\left(\omega_{1}\right), h\left(\omega_{1}\right), R\left(\omega_{1}\right)$ around $\hat{\omega}_{1}$ in powers of $n^{-\frac{1}{2}}$ and retaining terms only up to second order, as long as we restrict our attention to points satisfying $\left(\omega_{1}-\hat{\omega}_{1}\right) \sim O\left(n^{-\frac{1}{2}}\right)$. This property is worth emphasising, since the asymptotic results we will be deriving in this paper will be based on an expansion of the Laplace approximation to the marginal of $\omega_{1}$ rather than an expansion of the exact density itself, an approach which at first sight might suggest a further degradation of our results. The proposed replacement of the Laplace approximation by its Edgeworthtype expansion does not, however, retain its saddlepoint accuracy in the extreme tails and may be criticised on these grounds.

On the other hand, an excessive reliance on error rates as an operational assessment of accuracy is open to criticism itself, since it provides no specific bound for any particular data set - a point eloquently made by Hodges (1987) and Kass (1988). In our case, the pitfalls of an approach that judges procedures by focusing solely on their asymptotic error rates are highlighted by the lack of parameterisation invariance of the Laplace approximation: if we let $g\left(\omega_{2}\right)$ be a nonsingular transformation of the nuisance parameters, the Laplace approximation to the marginal distribution of $\omega_{1}$ based on the joint posterior of $\left(\omega_{1}, g\left(\omega_{2}\right)\right)$ will in general differ from that given in (2.12), depending on the form of $g$. So, one is faced with a multitude of Laplace approximations, some more accurate than others, but all having the same error rate! ${ }^{3}$ This unfortunate feature of the Laplace approximation is actually shared by other well-known modifications to the profile likelihood, such as the closely related conditional profile likelihood of Cox \& Reid (1987), though not by the modified profile likelihood of Barndorff-Nielsen (1983). The usefulness of the latter outside exponential and transformation models is somewhat limited and - more important to us - it does not have the justification as an approximation to a true Bayesian posterior that the Laplace approximation enjoys. In spite of the above, we feel that, for Bayesian problems requiring analytic solutions, the Laplace approximation provides a well understood and widely tested method that can be used with reasonable confidence in a variety of settings, especially problems where the posterior can be shown to possess a single interior mode.

An expansion of (2.12) around $\hat{\omega}_{1}$ allows us to establish the following relationships between the quantities appearing in (2.1) and those of (2.12):

$$
\begin{aligned}
V & =\left(\omega_{1}-\hat{\omega}_{1}\right)(-\ddot{R})^{+\frac{1}{2}} \\
d_{1} & =(\dot{w}-\dot{h} / 2)(-\ddot{R})^{-\frac{1}{2}} \\
d_{2} & =(\ddot{w}-\ddot{h} / 2)(-\ddot{R})^{-1} \\
F_{3} & =\dddot{R}(-\ddot{R})^{-\frac{3}{2}}
\end{aligned}
$$

\footnotetext{
${ }^{3}$ For an example see Tierney et al. $(1989$, sec. 3.3$)$
} 


$$
F_{4}=\dddot{R}(-\ddot{R})^{-2} \text {, }
$$

the dot notation denoting repeated differentiation and subsequent evaluation at the mode.

The provenance of the normalising transformation $r(V)$ in (2.6) is now apparent: since $-\frac{1}{2} r^{2}(v) \approx-\frac{1}{2} v^{2}+\frac{1}{6} F_{3} v^{3}+\frac{1}{24} F_{4} v^{4} \approx R(v)$ is but a second order expansion of the relative profile likelihood of the standardised variable $V$, we can think of (2.6) as a Bartlett-type correction of the normal approximation to the posterior distribution of $r(V)$, the signed square root of the likelihood ratio test statistic. The normalising transformation itself depends on the profile likelihood alone, but its mean and variance also involve the constants $d_{1}, d_{2}$, which in turn depend on the prior and determinant terms of the Laplace approximation. Setting $d_{1}=d_{2}=0$ in (2.4)-(2.11) allows us to easily gauge the effect of erroneously treating $-2 R(V)$ as a $\chi_{(1)}^{2}$ variable. The effect of prior or likelihood perturbations and the information adjustment for the nuisance parameters can also be isolated and interpreted through the effect of $d_{1}, d_{2}$ on the marginal density of $V$ directly. By differentiating the density approximation in (2.1) and evaluating at the profile mode $v=0$, we see that $d_{1}$ is a location parameter. It shifts the mode of $p_{V}(v)$ to the right of 0 , if positive, and to the left of 0 otherwise. Similarly $d_{2}$ can be interpreted as a scale parameter. It changes the observed information at $v=0$ from 1 to $\left(1-d_{2}\right)$, thus serving to flatten the profile likelihood, if positive, and sharpen its peak otherwise. This becomes clearer if we assume that $F_{3}=F_{4}=0$, in which case we recognise our approximation as a $N\left(\frac{d 1}{1-d_{2}},\left(1-d_{2}\right)^{-1}\right)$ kernel. The other two parameters - $F_{3}, F_{4}$ - depend only on the profile likelihood, and measure its departure from normal shape. $F_{4}$ increases tail weight if positive, and decreases it otherwise, while $F_{3}$ is a measure of skewness, redistributing weight from the left to the right tail if positive and vice versa.

If we then let $\hat{\pi}=\pi(\hat{\omega}), \dot{\pi}=\left.\frac{\partial \pi(\omega)}{\partial \omega}\right|_{\hat{\omega}}, \ddot{\Pi}=\left.\frac{\partial^{2} \pi(\omega)}{\partial \omega \partial \omega^{T}}\right|_{\hat{\omega}}$ and define

$$
\begin{aligned}
& \dddot{L}_{22} \dot{\omega}=\left.\sum_{k=1}^{p} \frac{\partial^{3} L(\omega)}{\partial \omega_{2} \partial \omega_{2}^{T} \partial \omega_{k}} \frac{\partial \hat{\omega}_{k}\left(\omega_{1}\right)}{\partial \omega_{1}}\right|_{\hat{\omega}}, \quad \dot{\omega}^{T} \dddot{L}_{2} \dot{\omega}=\left.\sum_{k=1}^{p} \sum_{l=1}^{p} \frac{\partial^{3} L(\omega)}{\partial \omega_{2} \partial \omega_{k} \partial \omega_{l}} \frac{\partial \hat{\omega}_{k}\left(\omega_{1}\right)}{\partial \omega_{1}} \frac{\partial \hat{\omega}_{l}\left(\omega_{1}\right)}{\partial \omega_{1}}\right|_{\hat{\omega}}, \\
& \dddot{L}_{22} \ddot{\omega}=\left.\sum_{k=1}^{p} \frac{\partial^{3} L(\omega)}{\partial \omega_{2} \partial \omega_{2}^{T} \partial \omega_{k}} \frac{\partial^{2} \hat{\omega}_{k}\left(\omega_{1}\right)}{\partial \omega_{1}^{2}}\right|_{\hat{\omega}}, \quad \dot{\omega}^{T} \dddot{L}_{22} \dot{\omega}=\left.\sum_{k=1}^{p} \sum_{l=1}^{p} \frac{\partial^{4} L(\omega)}{\partial \omega_{2} \partial \omega_{2}^{T} \partial \omega_{k} \partial \omega_{l}} \frac{\partial \hat{\omega}_{k}\left(\omega_{1}\right)}{\partial \omega_{1}} \frac{\partial \hat{\omega}_{l}\left(\omega_{1}\right)}{\partial \omega_{1}}\right|_{\hat{\omega}},
\end{aligned}
$$

it can be shown that

$$
\begin{aligned}
\ddot{R} & =\left(\ddot{L}^{11}\right)^{-1}, \\
\dddot{R} & =\dot{\omega}^{T}\left[\dot{\omega}^{T} \dddot{L} \dot{\omega}\right], \\
\dddot{R} & =\dot{\omega}^{T}\left[\dot{\omega}^{T} \dddot{L} \dot{\omega}\right] \dot{\omega}+3 \ddot{\omega}^{T}\left[\dot{\omega}^{T} \dddot{L} \dot{\omega}\right], \\
\dot{h} & =\operatorname{tr}\left(\ddot{L}_{22}^{-1}\left[\dddot{L}_{22} \dot{\omega}\right]\right), \\
\ddot{h} & =\operatorname{tr}\left(\ddot{L}_{22}^{-1}\left[\dot{\omega}^{T} \dddot{L}_{22} \dot{\omega}\right]+\ddot{L}_{22}^{-1}\left[\dddot{L}_{22} \ddot{\omega}\right]-\left(\ddot{L}_{22}^{-1}\left[\dddot{L}_{22} \dot{\omega}\right]\right)^{2}\right), \\
\dot{w} & =\frac{\dot{\pi}^{T} \dot{\omega}}{\hat{\pi}}, \\
\ddot{w} & =\frac{\dot{\pi}^{T} \ddot{\omega}}{\tilde{\pi}}+\dot{\omega}^{T}\left(\frac{\ddot{\pi}}{\pi}-\frac{\dot{\pi} \dot{\pi}^{T}}{\pi \tilde{\pi}}\right) \dot{\omega} .
\end{aligned}
$$


where we have used the convention that suppressing a subscript of a derivative array indicates differentiation w.r.t. to all the elements of $\omega$. Repeated differentiation of the identity $\frac{\partial L\left(\hat{\omega}\left(\omega_{1}\right)\right)}{\partial \omega_{2}} \equiv 0$ w.r.t. $\omega_{1}$, using the chain rule, shows that derivatives of the constrained likelihood mode $\hat{\omega}\left(\omega_{1}\right)$ reduce at $\hat{\omega}_{1}$ to

$$
\dot{\omega}=\left(\begin{array}{c}
1 \\
-\ddot{L}_{22}^{-1} \ddot{L}_{21}
\end{array}\right), \quad \ddot{\omega}=\left(\begin{array}{c}
0 \\
-\ddot{L}_{22}^{-1}\left[\dot{\omega}^{T} \dddot{L}_{2} \dot{\omega}\right]
\end{array}\right) \text {. }
$$

It is common to express the standardised version of $\omega_{1}$ in terms of the information matrix $I=-\ddot{L}$; we note that (2.21) implies $V=\left(\omega_{1}-\hat{\omega}_{1}\right)\left(I^{11}\right)^{-\frac{1}{2}}$.

Since we have amalgamated the effects of both the prior and the determinant term into $d_{1}, d_{2}$, our expansion of the Laplace posterior can be easily compared to Sprott's (1980) expansion of the profile likelihood, to which it reduces for $d_{1}=d_{2}=0$. It is obvious that, had the problem been univariate to start with, the posterior of $V$ would have had an expansion around $\hat{\omega}$ formally identical to (2.1), with $d_{1}, d_{2}$ depending on the prior alone. This Edgeworth-type expansion has received wide attention in the literature and approximations to its quantiles, tail probabilities, moments etc. have been obtained by a number of researchers. Indeed, our (2.4), (2.5) are corrected versions of (38), (39) of Welch \& Peers (1965). 4 Though their expansion is purely formal, it was later given a rigorous justification by Johnson (1970), further developed by Stein (1985) and recently used by Severini (1993). Peers (1965) also proposed tail probability approximations for a univariate parameter of interest in the presence of nuisance parameters, for which Nicolaou (1993) provided regularity conditions. Unlike ours, the expressions given by Peers \& Nicolaou are only first order correct, testimony to the difficulty of performing a multiple integration of the joint posterior. In expanding the Laplace approximation around $\hat{\omega}_{1}$ and then integrating the (univariate) asymptotic expansion, one trades a hard integration problem for a simpler differentiation one and can easily calculate the second order term that eluded them.

Another well-known approximation is that of Lindley (1980), also derived informally and known to contain errors. 5 In the univariate case, his expansion of the first four cumulants of $V$ is of a lower order of accuracy than ours, e.g. he set the kurtosis equal to 0 . Moreover, in the multivariate case, he did not tackle moments of order higher than the first. Where comparable, his approximations are numerically equivalent to ours, as can be readily shown using (2.28) below. Since he also derived his results using multiple integration of an expansion of the joint posterior around its unconstrained mode, while we performed a univariate integration of an expansion of the Laplace marginal around its constrained mode instead, the question arises as to which method is preferable. Though the answer might seem to be a question of taste, we are prepared to argue that our approach lends itself better to both interpretation and simplification. Ease of interpretation follows from the fact that both our tail, quantile and cumulant approximations are expressed in

\footnotetext{
'Their (38) contains an error: the coefficient of $v_{4}\left(-v_{2}\right)^{-2}$ should be $x^{3}+3 x$. The error is not propagated to eqn. 39 , which suggests that the error is simply a misprint.

${ }^{5}$ See Kass \& Steffey (1989, sec. 3.2).
} 
terms of the derivatives of the marginal rather than the joint posterior. Most statisticians can adequately gauge the significance of the first four derivatives of a univariate density, but find their intuition failing them when presented the derivative arrays of a multivariate density and asked to speculate as to the manner in which they affect univariate margins. As for amenability of our results to simplification, we hope to demonstrate it convincingly in the next section.

In order to check agreement with existing results expressed in terms of the observed information matrix, we need to use the matrix identity:

$$
\ddot{L}^{-1}=\left[\begin{array}{cc}
0 & 0^{T} \\
0 & \ddot{L}_{22}^{-1}
\end{array}\right]+\ddot{L}^{11} \dot{\omega} \dot{\omega}^{T},
$$

whence $\dot{\omega}=\ddot{L}^{1}\left(\ddot{L}^{11}\right)^{-1}$, where $\ddot{L}^{1}$ denotes the first column of $\ddot{L}^{-1}$. Equation (2.28) also implies that, for any $p \times p$ symmetric matrix $C$ partitioned conformably with $\ddot{L}$,

$$
\operatorname{tr}\left[\ddot{L}^{-1}-\ddot{L}^{11} \dot{\omega} \dot{\omega}^{T}\right]\left[\begin{array}{ll}
C_{11} & C_{12} \\
C_{21} & C_{22}
\end{array}\right]=\operatorname{tr}\left[\begin{array}{cc}
0 & 0^{T} \\
0 & \ddot{L}_{22}^{-1}
\end{array}\right]\left[\begin{array}{ll}
C_{11} & C_{12} \\
C_{21} & C_{22}
\end{array}\right]=\operatorname{tr}\left(\ddot{L}_{22}^{-1} C_{22}\right)
$$

This allows us to increase dimension and reconcile our expressions to those of the aforementioned authors, where applicable.

Our approximation to the cumulant generating function of $V$ is based on a recent paper by Fraser \& Reid (1993). In that paper they derive the cumulant generating function of $V$ under a flat prior and no nuisance parameters. As expected, our expressions reduce to theirs for $d_{1}=d_{2}=0$. They also fail to provide regularity conditions, but instead refer to previous work by Hinkley (1978). However, the details are actually to be found in Efron \& Hinkley (1978).

In fitting standard densities by matching derivatives, we were motivated by the work of Viveros \& Sprott (1987), which built on Sprott's (1980) suggestion to fit Student- $t$ densities to profile likelihoods that exhibit negligible skewness and suggested a $\log \mathrm{F}$ approximation for the case where neither skewness nor kurtosis could be ignored. In the context of locationscale models with Gumbel errors and possible censoring, they then examined the distribution of the pivots conditional on a set of ancillaries and showed that the profile likelihood alone provided satisfactory approximations for inference on the location parameter, but not for the scale parameter. Finally they showed that a saddlepoint approximation to the exact conditional distributions led to a marked improvement in the fit and could be expanded to provide the parameters of a $\log F$ density. The expansion they considered is of the form given in (2.1) but with $d_{2}=0$, a simplifying assumption which we have dropped. We were intrigued by their results and noted the formal equivalence of the saddlepoint approximation to the conditional distribution of the pivot given the ancillaries to the Laplace posterior of the pivot under a flat prior. ${ }^{6}$ Both appear in a simplified form in location-scale models, since there is a single nuisance parameter and, therefore, the determinant term is trivial.

\footnotetext{
${ }^{6}$ For another instance of conditional inference based on a modified profile likelihood being formally equivalent to Bayesian inference using a Laplace posterior see Sweeting (1987) .
} 
In later sections we shall examine the extent to which these results can be generalised to censored regression models.

The asymptotic equivalence of (2.5) and (2.6) allows us to compare our results to those provided by DiCiccio et al. (1990) for $r(V)$, the signed square root of the likelihood ratio. In (7)-(11) of that paper they expanded $r(V)$ in terms of $V\left(\omega_{1}\right)$ and derived its first two posterior moments under a flat prior. They subsequently used its standardised version as an approximate normal pivot, a result at which we arrived via a different route: an approximation to the density of $V$ itself and the Welch \& Peers' expressions. They then expressed their results in terms of $\omega$ itself and showed that (2.6) is asymptotically equivalent to a formula that only involves first and second derivatives of the posterior of $\omega$ evaluated at the constrained posterior mode $\hat{\omega}\left(\omega_{1}\right)$. The price paid for not having to calculate third and fourth derivatives, as we do, is twofold: (i) evaluation of the tail probability $P[V \leq$ v] requires a separate constrained maximisation at each $\omega_{1}$ and (ii) their results can be seriously in error when the marginal posterior mode differs significantly from the profile posterior mode, as they admit. In an independent application of their formulae, Kass \& Slate (1992) also reported difficulties in attaining convergence in the constrained maximisations above, when extreme tail probabilities where involved. DiCiccio et al. (1990) attempted to remedy those problems by providing a second formula which requires the first and second derivatives of the Laplace posterior itself and showed that it overcomes the difficulties associated with their earlier result. Their normal example, though, is artificially simple in that the derivatives of the Laplace approximation will not in general be available in closed form. Approximating them numerically by finite differences is not a real option either, since each function evaluation requires a separate constrained maximisation.

DiCiccio \& Field (1991) recognised this difficulty and suggested a reparameterisation based on the observation that, under flat priors, the Laplace posterior mode coincides with the profile mode if the determinant term is constant to second order, i.e. the information about the nuisance parameters changes slowly with the parameter of interest. Though the reparameterisation to achieve this is not available in general, it can be given in closed form for the scale parameter of a normal regression model with no censoring, to which their examples pertain. They then proceeded by analogy and showed that the same reparameterisation also gives accurate results for the scale parameter of regression model with $t_{(5)}$ and Gumbel errors. Their results seem quite convincing in this particular case, but do not generalise easily to other settings.

We have not used the approximations given in the previous two papers. Instead we noticed that (i) they were essentially attempts to sidestep the problem of directly integrating an expansion of the Laplace posterior, (ii) they pertain to individual tail probabilities, not the entire posterior distribution and (iii) most of the examples to which they were applied were actually drawn from regression models with possible censoring. We shall show in the next section that such models possess special structure that allows us to face the problem head on and replace fifth and sixth order sums of the likelihood derivatives with double sums at the most, while at the same time gaining insights about the quantities of interest 
that cannot be provided by a purely numerical approximation.

\section{Censored Linear Regression Models}

We shall assume that a sample of $n$ observations $u_{1}, \ldots, u_{n}$ is available from the regression model

$$
U_{i}=\theta_{0}+w_{i}^{T} \theta_{1}+Z_{i} \exp \left(\theta_{2}\right), \quad Z_{i} \stackrel{i i d}{\sim} F_{Z}(z)
$$

with the first $m$ fully observed, the remaining $n-m$ having been right-censored. Here $\left\{u_{i}\right\}_{i=1}^{n}$ denote the values actually recorded, be they censored or not. If the data are loglifetimes, $\left\{u_{i}\right\}$ is but the logarithm of the time under observation for the $i$ 'th case. At this point we shall make no further assumptions about the type of censoring mechanism, other than to require that it carries no information about the parameters. We shall treat the covariates as constants measured without error and assume that $F_{Z}(z)$ is a known distribution on the real line.

In the current parameterisation $\theta_{0} \in \Re^{1}$ is the intercept, $\theta_{1} \in \Re^{k-1}$ is a vector of regression slopes and $\theta_{2} \in \Re^{1}$ is the logarithm of the scale parameter, constituting the set of parameters denoted by $\theta$. A restriction of our model - implicit in the parameterisation above - is that we do not allow regression through the origin. As we shall see later in this section, including the intercept in the model greatly simplifies the approximation of quantile marginal densities, since we can express any quantile as the intercept of an appropriately transformed regression model.

Under a flat prior on $\theta$, the posterior is given by :

$$
p(\theta ; u) \propto \exp \left(-m \theta_{2}\right) \prod_{i=1}^{m} f_{Z}\left(z_{i}(\theta)\right) \prod_{i=m+1}^{n}\left[1-F_{Z}\left(z_{i}(\theta)\right)\right]
$$

where

$$
z_{i}(\theta)=\left(u_{i}-\theta_{0}-w_{i}^{T} \theta_{1}\right) \exp \left(-\theta_{2}\right)
$$

If we define

$$
g\left(z_{i}\right) \equiv \begin{cases}-\log f_{Z}\left(z_{i}\right), & i=1 \ldots m, \\ -\log \left[1-F_{Z}\left(z_{i}\right)\right], & i=m+1 \ldots n,\end{cases}
$$

to be minus the loglikelihood contribution of the $i$ 'th case, we can express the logposterior as :

$$
\log p(\theta ; u)=L(\theta)=c-m \theta_{2}-\sum_{i=1}^{n} g\left[z_{i}(\theta)\right]
$$

where $c$ denotes a generic constant. 
Since we are primarily interested in lifetime data, for which left censoring is rather rare, we have only considered right censoring. However, all our subsequent results will be based not on (3.2) but on (3.5), a form which allows us to suppress the dependence of $L(\theta)$ on the particular error density and the censoring pattern. It should then be relatively easy to extend our results to handle left censoring by letting $g(z)$ in (3.4) equal $-\log \left[F_{Z}(z)\right]$ for left censored observations.

In censored regression models it is often the case that, in addition to the regression coefficients and the scale parameter, interest centers on certain quantiles of the lifetime distribution, the survivor function as well as the distribution function of extreme order statistics of future samples. To simplify approximations to their marginal posteriors, we propose reparameterising $p(\theta)$ in either of two ways, according to the problem at hand.

\subsection{Parameterisation I}

Our first reparameterisation assumes that interest centers on the regression coefficients, the scale parameter or an arbitrary quantile of the response. If we let $z_{p}$ denote the $p^{\prime}$ th quantile of $F_{Z}(z)$ and $w$ a covariate vector of interest, then the $p$ 'th quantile of $F_{U}(u ; \theta)$ is

given by $u_{p}(\theta)=\theta_{0}+w^{T} \theta_{1}+z_{p} \exp \left(\theta_{2}\right)$. It follows that obtaining an approximation to the posterior distribution of an arbitrary quantile dispenses with the need to derive a separate approximation for the intercept, which is seen from above to be the quantile that has $w=0, z_{p}=0$. For $p$ in $(0,1)$ we adopt the following data-dependent reparameterisation: $\gamma_{0}=\left(u_{p}(\theta)-u_{p}(\hat{\theta})\right) \exp \left(-\hat{\theta}_{2}\right), \gamma_{1}=\left(\theta_{1}-\hat{\theta}_{1}\right) \exp \left(-\hat{\theta}_{2}\right), \delta=\left(\theta_{2}-\hat{\theta}_{2}\right)$. On setting $\omega^{T}=\left(\gamma^{T}, \delta\right)$ and $\hat{z}_{i}=z_{i}(\hat{\theta})$, the logposterior transforms to:

$$
\log p(\omega) \equiv L(\omega)=c-m \delta-\sum_{i=1}^{n} g\left[z_{i}(\omega)\right]
$$

where

$$
\begin{aligned}
& p_{i}(\omega)=\left[\hat{z}_{i}-z_{p}-\gamma_{0}-\left(w_{i}-w\right)^{T} \gamma_{1}\right] \exp (-\delta) \\
& z_{i}(\omega)=p_{i}(\omega)+z_{p} .
\end{aligned}
$$

It is worth noting that a flat prior and a constant Jacobian imply that our posterior remains proportional to the likelihood after reparameterisation and, therefore, the posterior mode is invariant: $\hat{\omega}=\omega(\hat{\theta})=0$. When $\hat{\theta}$ is known, the joint posterior of the standardised $p$ 'th quantile, the regression slopes and logscale parameter can be obtained by simply recentering $\hat{z}$ and the design matrix $W$, no further maximisations being necessary.

In the absence of censoring, $\omega$ is a pivotal quantity whose frequentist distribution, conditional on the vector of ancillaries $\hat{z}$, coincides with its Bayesian posterior under a flat prior. For location-scale models it is shown in Bogdanoff \& Pierce (1973) and Lawless (1982) that this property holds even for censored data, provided the censoring mechanism is of Type II, i.e. the experiment is terminated after a fixed number of observations has become available. Since a flat prior in this case reproduces conditional frequentist inferences, we may call it 
'noninformative'. It differs from Jeffreys' prior, which turns out to depend on the design matrix and requires more detailed assumptions about the censoring mechanism.

The function $g(z)$ was set equal to minus the loglikelihood contribution of an individual case, so that, for censored observations, $g^{\prime}(z)$ equals $h(z)$, the hazard function of $Z$. This allows us to express higher order derivatives of $g(z)$ in terms of $h(z)$ and its derivatives, well-studied quantities for common distributions. Since $g^{\prime \prime}(z)$ appears very frequently in later work, we note from above that it will be nonnegative for logconcave densities with nondecreasing hazard functions. With those densities in mind, we define for $i=1 \ldots n$ :

$$
p_{2 i}=\frac{g^{\prime \prime}\left(\hat{z}_{i}\right)}{\sum_{k=1}^{n} g^{\prime \prime}\left(\hat{z}_{k}\right)}, \quad p_{3 i}=\frac{g^{\prime \prime \prime}\left(\hat{z}_{i}\right)}{\sum_{k=1}^{n} g^{\prime \prime}\left(\hat{z}_{k}\right)}, \quad p_{4 i}=\frac{g^{\prime \nu}\left(\hat{z}_{i}\right)}{\sum_{k=1}^{n} g^{\prime \prime}\left(\hat{z}_{k}\right)}
$$

In this context, $\left\{p_{2 i}\right\}_{i=1}^{n}$ can be thought of as defining a data-dependent probability distribution, supported on the $n$ sample points. Since we condition on the data, that distribution is fixed. In the remainder of this work we formally define the 'expectation' of a generic function $\phi=\left\{\phi_{i}\right\}_{i=1}^{n}$ by

$$
E[\phi] \equiv \sum_{i=1}^{n} p_{2 i} \phi_{i}
$$

The introduction of this pseudo-expectation will not only simplify our formulae, but will also enhance the interpretability of our expansions by bringing out analogies with well known regression results.

The nature of $\left\{p_{3 i}\right\}_{i=1}^{n},\left\{p_{4 i}\right\}_{i=1}^{n}$ is not as clear, but it is shown in sec. 4 that $p_{4 i} \propto p_{3 i} \propto$ $p_{2 i}$ for some distributions of interest. In that case, $\left\{p_{3 i}\right\}_{i=1}^{n},\left\{p_{4 i}\right\}_{i=1}^{n}$ can be thought of as positive finite measures, assigning weight $p_{3 i}, p_{4 i}$ respectively to the $i$ 'th sample point. The common divisor of the fractions in (3.8), $\tilde{n}=\sum_{k=1}^{n} g^{\prime \prime}\left(\hat{z}_{k}\right)$, is the rate at which the sample information grows. We have assumed it to be $O(n)$, an assumption whose validity depends on the censoring pattern.

\section{Quantiles and individual regression coefficients}

In this section we shall assume that $\gamma^{T}$ has been partitioned into $\left(\alpha, \beta^{T}\right)$, where $\alpha$ is the single parameter of interest and $\beta$ is a vector of nuisance parameters. Here $\alpha$ may be an arbitrary quantile or a slope parameter, depending on the centering employed and whether or not the columns of the design matrix have been permuted. To approximate the marginal of the $p^{\prime}$ th quantile at a particular design point $w$, we would let $x_{i}^{T}=\left[1,\left(w_{i}-w\right)^{T}\right]$ and $\hat{p}_{i}=\hat{z}_{i}-z_{p}$, where $z_{p}=F_{Z}^{-1}(p)$. Setting $z_{p}=0$ would return the location parameter for observations with covariate vector equal to $w$. If in addition $w$ were set to zero, the marginal of the intercept would be obtained. Finally, if one of the regression slopes were of interest, we would set $\hat{p}_{i}=\hat{z}_{i}-E[\hat{z}]$ and let $x_{i}^{T}$ be the permutation of the elements of $\left[1,\left(w_{i}-E[w]\right)^{T}\right]$ that makes the first column of the design matrix $X$ correspond to the variable of interest. Whatever the final form of $X$, we would then partition $x_{i}^{T}$ into $\left[v_{i}, y_{i}^{T}\right]$, 
set $\psi_{i}^{T}=\left[y_{i}^{T}, \hat{p}_{i}\right]$ and calculate, for $i, j=1 \ldots n$, the scalars :

$$
\begin{aligned}
r & =m / \tilde{n}, \quad\left\langle y_{i}, y_{j}\right\rangle=y_{i}^{T} E\left[y y^{T}\right]^{-1} y_{j}, \quad \tilde{p}_{i}=y_{i}^{T} E\left[y y^{T}\right]^{-1} E[y \hat{p}], \quad e_{p \cdot y i}=\hat{p}_{i}-\tilde{p}_{i}, \\
\tilde{v}_{y i} & =y_{i}^{T} E\left[y y^{T}\right]^{-1} E[y v], \quad e_{v \cdot y i}=v_{i}-\tilde{v}_{y i}, \quad\left\langle\psi_{i}, \psi_{j}>=\left\langle y_{i}, y_{j}>+\frac{e_{p \cdot y i} e_{p \cdot y j}}{r+E\left[e_{p \cdot y}^{2}\right]},\right.\right. \\
\delta_{\alpha} & =-\frac{E\left[e_{v \cdot y} e_{p \cdot y}\right]}{r+E\left[e_{p \cdot y}^{2}\right]}, \quad e_{\alpha i}=e_{v \cdot y i}+\delta_{\alpha} e_{p \cdot y i}, \quad \delta_{\alpha \alpha}=\frac{\sum_{i=1}^{n}\left(p_{3 i} e_{p \cdot y i}+p_{2 i} 2\right) e_{\alpha i}^{2}}{r+E\left[e_{p \cdot y}^{2}\right]}-\delta_{\alpha}^{2}, \\
\tilde{e}_{\alpha i}^{2} & =\sum_{j=1}^{n} p_{3 j} e_{\alpha j}^{2}\left\langle y_{j}, y_{i}>, \quad \hat{e}_{\alpha i}=\frac{E\left[e_{\alpha} e_{p \cdot y}\right]}{r+E\left[e_{p \cdot y}^{2}\right]} e_{p \cdot y i} .\right.
\end{aligned}
$$

By definition, $\tilde{v}_{i}=\sum_{j=1}^{n} p_{2 j} v_{j}<y_{j}, y_{i}>, \tilde{p}_{i}=\sum_{j=1}^{n} p_{2 j} \hat{p}_{j}<y_{j}, y_{i}>$ are the linear regressions of $v, p$ on $y$ and $e_{p \cdot y}, e_{p \cdot y}$ have an obvious interpretation as residuals. It would also appear that $e_{\alpha}$ is the residual of the regression of $v$ on $\psi$, but actually $e_{\alpha}$ is uncorrelated only with $y$ and not with the residuals of $\hat{p}$ on $y$. The reason is that $\delta_{\alpha}$ is only proportional to the regression coefficient of $v$ on $e_{p \cdot y}$, the ratio tending to 1 as $E\left[e_{p \cdot y}^{2}\right]$ increases or $r$ tends to zero. This is more easily seen is we assume that $E\left[e_{p \cdot y}^{2}\right]>0$ and rewrite $e_{\alpha i}$ as

$$
e_{\alpha i}=e_{v \cdot y i}+\delta_{\alpha} e_{p \cdot y i}=e_{v \cdot y i}-\frac{E\left[e_{p \cdot y}^{2}\right]}{r+E\left[e_{p \cdot y}^{2}\right]} \frac{E\left[e_{v \cdot y} e_{p \cdot y}\right]}{E\left[e_{p \cdot y}^{2}\right]} e_{p \cdot y i}
$$

Here $\bar{e}_{\alpha i}^{2}$ may be thought of as the linear fit of $e_{\alpha i}^{2}$ on $y$, for the special case $p_{2} \propto p_{3}$. Finally, $\hat{e}_{\alpha i}$ is $\frac{E\left[e_{p \cdot y}^{2}\right]}{r+E\left[e_{p \cdot y}^{2}\right]}$ times the projection of $e_{\alpha i}$ on $e_{p \cdot y i}$.

The constants of (2.16)-(2.20) would then be determined by:

$$
\begin{aligned}
& \frac{\partial^{2} R}{\partial \alpha^{2}}=-\tilde{n}\left(\sum_{i=1}^{n}\left[p_{2 i} e_{\alpha i}^{2}\right]+r \delta_{\alpha}^{2}\right) \\
& \frac{\partial^{3} R}{\partial \alpha^{3}}=+\tilde{n}\left(\sum_{i=1}^{n}\left[p_{3 i} e_{\alpha i}^{3}+p_{2 i} 3 e_{\alpha i}\left(2 e_{\alpha i}-\hat{p}_{i} \delta_{\alpha}\right) \delta_{\alpha}\right]+r \delta_{\alpha}^{3}\right), \\
& \frac{\partial^{4} R}{\partial \alpha^{4}}=-\tilde{n}\left(\sum _ { i = 1 } ^ { n } \left[p_{4 i} e_{\alpha i}^{4}+p_{3 i} 6 e_{\alpha i}^{2}\left(2 e_{\alpha i}-e_{p \cdot y i} \delta_{\alpha}\right) \delta_{\alpha}+\right.\right. \\
&+3 \tilde{n}\left(\sum_{i=1}^{n}\left[p_{3 i} e_{\alpha i}^{2} \tilde{e}_{\alpha i}^{2}\right]+\delta_{\alpha \alpha}^{2}\left(\sum_{i=1}^{n}\left[p_{2 i} e_{p \cdot y i}^{2}\right]+r\right)\right), \\
&\left.\left.\left.\frac{\partial h}{\partial \alpha}=-\sum_{i=1}^{n}\left[p_{3 i}^{2} e_{\alpha i}+p_{2 i} 2 \delta_{\alpha}\right]<\psi_{i}, \psi_{i}>+2 \delta_{\alpha i}, \hat{p}_{i} \delta_{\alpha}+3 e_{p \cdot y i}^{2} \delta_{\alpha}^{2}\right) \delta_{\alpha}^{2}\right]+r \delta_{\alpha}^{4}\right)
\end{aligned}
$$




$$
\begin{aligned}
\frac{\partial^{2} h}{\partial \alpha^{2}}= & \left.+\sum_{i=1}^{n}\left[\left(p_{4 i} e_{\alpha i}{ }^{2}-p_{3 i} \tilde{e}_{\alpha i}^{2}\right)+p_{3 i}\left(2 e_{\alpha i}-e_{p \cdot y i} \delta_{\alpha}\right) \delta_{\alpha}\right]<\psi_{i}, \psi_{i}\right\rangle \\
& \left.-\sum_{i=1}^{n} \sum_{j=1}^{n}\left[p_{3 i} p_{3 j} e_{\alpha i} e_{\alpha j}\right]<\psi_{i}, \psi_{j}><\psi_{i}, \psi_{j}\right\rangle \\
& \left.-\delta_{\alpha \alpha} \sum_{i=1}^{n}\left[p_{3 i} e_{p \cdot y i}+p_{2 i} 2\right]<\psi_{i}, \psi_{i}\right\rangle \\
& +\frac{1}{r+E\left[e_{p \cdot y}^{2}\right]} \sum_{i=1}^{n}\left[p_{3 i} 4 e_{\alpha i}\left(e_{\alpha i}-\hat{e}_{\alpha i}\right) e_{p \cdot y i}+p_{2 i} 2\left(e_{\alpha i}^{2}-e_{p \cdot y i}^{2} \delta_{\alpha}^{2}\right)\right] \\
& -4 \delta_{\alpha}^{2}\left(\frac{r}{r+E\left[e_{p \cdot y}^{2}\right]}\right)\left(1+\frac{r}{r+E\left[e_{p \cdot y}^{2}\right]}\right) .
\end{aligned}
$$

Under our flat prior, both $\frac{\partial w}{\partial \alpha}$ and $\frac{\partial^{2} w}{\partial \alpha^{2}}$ of (2.17), (2.18) are identically 0 . If a different prior was entertained, the expressions in (2.26), (2.27) would also have to be evaluated.

\section{The scale parameter}

In order to simplify the expansion of the marginal distribution of the logscale parameter, we would first perform a data-dependent centering of the standardised residuals $\hat{z}$ and the covariate matrix $W$, by setting $\hat{p}_{i}=\hat{z}_{i}-E[\hat{z}]$ and $x_{i}^{T}=\left[1, y_{i}^{T}\right]=\left[1,\left(w_{i}-E[w]\right)^{T}\right]$. This is the choice of centering which, in the previous section, would have turned $\alpha$ into the quantile orthogonal to $(\beta, \delta)$ and made the submatrix of the Hessian corresponding to $(\beta, \delta)$ a rank-1 modification of the covariance matrix of $\left(y^{T}, \hat{p}\right)$ w.r.t. the measure defined in (3.9). For $i, j=1 \ldots n$ we would then calculate the scalars:

$$
\begin{gathered}
r=m / \tilde{n}, \quad<y_{i}, y_{j}>=y_{i}^{T} \Sigma_{Y Y}^{-1} y_{j}, \quad \tilde{p}_{i}=y_{i}^{T} \Sigma_{Y Y}^{-1} \sigma_{Y P}, \quad e_{\delta i}=\hat{p}_{i}-\tilde{p}_{i}, \\
<x_{i}, x_{j}>=1+<y_{i}, y_{j}>, \quad \alpha_{\delta \delta}=\sum_{j=1}^{n} p_{3 j} e_{\delta j}^{2}, \quad \tilde{e}_{\delta i}^{2}=\sum_{j=1}^{n} p_{3 j} e_{\delta j}^{2}<y_{j}, y_{i}>.
\end{gathered}
$$

Here $\tilde{p}_{i}=\sum_{j=1}^{n} p_{2 j} \hat{p}_{j}<y_{j}, y_{i}>$ is the linear regression of $\hat{p}_{i}$ on $y_{i}$, which coincides with that of $\hat{p}_{i}$ on $x_{i}$, since $\mathrm{E}[\hat{p}]=0$. The interpretation of $e_{\delta}$ as a residual is then immediate. Both $\alpha_{\delta \delta}, \tilde{e}_{\delta}^{2}$ involve the squared residuals $e_{\delta}^{2}$, being proportional to their mean and linear regression on $y$ respectively, for the special case $p_{2} \propto p_{3}$. An interpretation for $r$ follows by recalling that $m$ is the number of fully observed cases in our sample and $\tilde{n}$ is the effective sample size. The constants of (2.16)-(2.20) and hence of (2.1) would then be determined by :

$$
\frac{\partial^{2} R}{\partial \delta^{2}}=-\bar{n}\left(\sum_{i=1}^{n}\left[p_{2 i} e_{\delta i}^{2}\right]+r\right)
$$




$$
\begin{aligned}
\frac{\partial^{3} R}{\partial \delta^{3}}= & +\tilde{n}\left(\sum_{i=1}^{n}\left[p_{3 i} e_{\delta i}^{3}+p_{2 i} 3 e_{\delta i}^{2}\right]+r\right) \\
\frac{\partial^{4} R}{\partial \delta^{4}}= & -\tilde{n}\left(\sum_{i=1}^{n}\left[p_{4 i} e_{\delta i}^{4}+p_{3 i} 6 e_{\delta i}^{3}+p_{2 i} 7 e_{\delta i}^{2}\right]+r\right) \\
& +3 \tilde{n}\left(\sum_{i=1}^{n}\left[p_{3 i} e_{\delta i}^{2} \tilde{e}_{\delta i}^{2}\right]+\alpha_{\delta \delta}^{2}\right) \\
\frac{\partial h}{\partial \delta}= & -\sum_{i=1}^{n}\left[p_{3 i} e_{\delta i}+p_{2 i} 2\right]<x_{i}, x_{i}> \\
\frac{\partial^{2} h}{\partial \delta^{2}}= & +\sum_{i=1}^{n}\left[\left(p_{4 i} e_{\delta i}^{2}-p_{3 i} \tilde{e}_{\delta i}^{2}\right)+p_{3 i} e_{\delta i}\right]<x_{i}, x_{i}> \\
& -\sum_{i=1}^{n} \sum_{j=1}^{n}\left[p_{3 i} p_{3 j} e_{\delta i} e_{\delta j}\right]<x_{i}, x_{j}><x_{i}, x_{j}>
\end{aligned}
$$

Since a flat prior is assumed, both $\frac{\partial w}{\partial \delta}$ and $\frac{\partial^{2} w}{\partial \delta^{2}}$ of $(2.17),(2.18)$ are identically 0 .

\subsection{Parameterisation II}

Here we shall assume that we are observing lifetime data and that interest centers on $S_{U}(\theta ; t, w)$, the survival probability beyond $u_{t}=\log t$ for a future observation whose covariate vector is $w$. Suppressing the dependence on $w$, it follows from (3.1) that the survivor function of $U$ satisfies $S_{U}(\theta ; t)=1-F_{Z}\left[z_{t}(\theta)\right]$, where $z_{t}(\theta)=\left(u_{t}-\theta_{0}-w^{T} \theta_{1}\right) \exp \left(-\theta_{2}\right)$. Assuming $f_{Z}(z)$ is strictly positive, $S_{U}(\theta ; t)$ and, hence, the distribution functions of the minimum or maximum of $l$ future observations at the same design point $w$ are 1-1 functions of $z_{t}$, whose distribution we propose to approximate instead. It is often the case that $z_{t}$ may be of interest in itself. If, for example, we let $u_{t}$ to be one of the observed $u$ 's in our sample, then $z_{t}$ is a 'realised error' from our model and its marginal distribution has been used to check model assumptions, see Zellner (1975) and Chaloner \& Brant (1988).

Initially, we reparameterise to $\xi_{0}=-z_{t}(\theta), \xi_{1}=\theta_{1}, \xi_{2}=\theta_{2}$. Since $\left|\frac{\partial \theta}{\partial \xi}\right|=\exp \left(\xi_{2}\right)$, the Jacobian cannot be ignored in this case and the posterior mode does not remain invariant under reparameterisation. From (3.3), (3.4), (3.5), it follows that the posterior of $\xi$ is given by :

$$
\log p(\xi ; u)=c-(m-1) \xi_{2}-\sum_{i=1}^{n} g\left[z_{i}(\xi)\right]
$$

where $z_{i}=z_{i}(\xi)=\left[\left(u_{i}-u_{t}\right)-\left(w_{i}-w\right)^{T} \xi_{1}\right] \exp \left(-\xi_{2}\right)-\xi_{0}$. We let $\hat{\xi}$ be the mode of $(3.20)$ and set $\hat{z}_{i}=z_{i}(\hat{\xi})$. If $m$ is not too small, the posterior mode $\hat{\xi}$ will be only a few iterations away from the MLE $\xi(\hat{\theta})$. Since $\hat{\theta}$ will in general be available from an earlier stage of our analysis, we can initialise any maximisation algorithm very close to the posterior mode and obtain rapid convergence. 
We then use the reparameterisation $\alpha=\left(\xi_{0}-\hat{\xi}_{0}\right), \beta=\left(\xi_{1}-\hat{\xi}_{1}\right) \exp \left(-\hat{\xi}_{2}\right), \delta=\left(\xi_{2}-\hat{\xi}_{2}\right)$, which relocates the posterior mode to the origin. With $\omega^{T}=\left(\alpha, \beta^{T}, \delta\right)$ and $\hat{z}_{t}=-\hat{\xi}_{0}$, we can rewrite the logposterior as:

$$
\log p(\omega ; u) \equiv L(\omega)=c-(m-1) \delta-\sum_{i=1}^{n} g\left[z_{i}(\omega)\right]
$$

where

$$
\begin{aligned}
& p_{i}(\omega)=\left[\hat{z}_{i}-\hat{z}_{t}-\left(w_{i}-w\right)^{T} \beta\right] \exp (-\delta) \\
& z_{i}(\omega)=p_{i}(\omega)+\hat{z}_{t}-\alpha_{i}
\end{aligned}
$$

This is the parameterisation we shall be using in our expansions, though the $\xi$ parameterisation is needed for the maximisation step. Comparing (3.21), (3.22) with (3.6), (3.7) we see that $m$ has been replaced by $m-1$ and $p_{i}, z_{i}$ have been redefined and are now calculated at the new posterior mode. If we now center the covariates by setting $y_{i}=w_{i}-w$, we find that the derivatives of $\log p(\omega ; u)$ w.r.t. $\beta, \delta$ alone agree at $\hat{\omega}=0$ with those obtained previously, provided we change $m$ to $m-1$. All the derivatives w.r.t. to $\alpha$ change, however, and this forces us to recalculate many quantities of interest.

\section{The survivor function and realised errors}

We can now approximate the marginal of the error $\alpha=\hat{z}_{t}-z_{t}(\theta)$, which is to be used to derive credible intervals for $S_{U}(\theta ; t)=1-F_{Z}\left(\hat{z}_{t}-\alpha\right)$, a $1-1$ function of $\alpha$ when the error density is strictly positive. For $i, j=1 \ldots n$ and with $v_{i}=1$, we calculate the scalars :

$$
\begin{aligned}
r & =(m-1) / \tilde{n},\left\langle y_{i}, y_{j}>=y_{i}^{T} E\left[y y^{T}\right]^{-1} y_{j}, \tilde{p}_{i}=y_{i}^{T} E\left[y y^{T}\right]^{-1} E[y \hat{p}], e_{p \cdot y i}=\hat{p}_{i}-\tilde{p}_{i},\right. \\
\tilde{v}_{y i} & =y_{i}^{T} E\left[y y^{T}\right]^{-1} E[y v], \quad e_{v \cdot y i}=v_{i}-\tilde{v}_{y i},<\psi_{i}, \psi_{j}>=<y_{i}, y_{j}>+\frac{e_{p \cdot y i} e_{p \cdot y j}}{r+E\left[e_{p \cdot y}^{2}\right]}, \\
\delta_{\alpha} & =-\frac{E\left[e_{v \cdot y} e_{p \cdot y}\right]}{r+E\left[e_{p \cdot y}^{2}\right]}, \quad e_{\alpha i}=e_{v \cdot y i}+\delta_{\alpha} e_{p \cdot y i}, \delta_{\alpha \alpha}=\frac{\sum_{i=1}^{n} p_{3 i} e_{p \cdot y i} e_{\alpha i}^{2}}{r+E\left[e_{p \cdot y}^{2}\right]}+\delta_{\alpha}^{2}\left(1-\frac{2 r}{r+E\left[e_{p \cdot y}^{2}\right]}\right), \\
\tilde{e}_{\alpha i}^{2} & =\sum_{j=1}^{n} p_{3 j} e_{\alpha j}^{2}\left\langle y_{j}, y_{i}>, \hat{e}_{\alpha i}=\frac{E\left[e_{\alpha} e_{p \cdot y}\right]}{r+E\left[e_{p \cdot y}^{2}\right]} e_{p \cdot y i} .\right.
\end{aligned}
$$

We see that the quantities that need to be calculated are the same as those needed for the quantiles. However, $r, \delta_{\alpha \alpha}$ have been redefined. The constants of (2.16)-(2.20) are determined by :

$$
\frac{\partial^{2} R}{\partial \alpha^{2}}=-\tilde{n}\left(\sum_{i=1}^{n}\left[p_{2 i} e_{\alpha i}^{2}\right]+r \delta_{\alpha}^{2}\right)
$$




$$
\begin{aligned}
& \frac{\partial^{3} R}{\partial \alpha^{3}}=+\tilde{n}\left(\sum_{i=1}^{n}\left[p_{3 i} e_{\alpha i}^{3}+p_{2 i} 3 e_{\alpha i}\left(2 e_{\alpha i}-\hat{p}_{i} \delta_{\alpha}-2\right) \delta_{\alpha}\right]+r \delta_{\alpha}^{3}\right), \\
& \frac{\partial^{4} R}{\partial \alpha^{4}}=-\tilde{n}\left(\sum_{i=1}^{n}\left[p_{4 i} e_{\alpha i}^{4}+p_{3 i} 6 e_{\alpha i}^{2} e_{p \cdot y i} \delta_{\alpha}^{2}+p_{2 i}\left(4 e_{\alpha i}+3 e_{p \cdot y i} \delta_{\alpha}\right) e_{p \cdot y i} \delta_{\alpha}^{3}\right]+r \delta_{\alpha}^{4}\right) \\
& +3 \tilde{n}\left(\sum_{i=1}^{n}\left[p_{3 i} e_{\alpha i}^{2} e_{\alpha i}^{2}\right]+\delta_{\alpha \alpha}^{2}\left(\sum_{i=1}^{n}\left[p_{2 i} e_{p \cdot y i}^{2}\right]+r\right)\right), \\
& \left.\frac{\partial h}{\partial \alpha}=-\sum_{i=1}^{n}\left[p_{3 i} e_{\alpha i}+p_{2 i} 2 \delta_{\alpha}\right]<\psi_{i}, \psi_{i}\right\rangle \text {, } \\
& \frac{\partial^{2} h}{\partial \alpha^{2}}=+\sum_{i=1}^{n}\left[\left(p_{4 i} e_{\alpha i}^{2}-p_{3 i} \tilde{e}_{\alpha i}^{2}\right)+p_{3 i} e_{p \cdot y i} \delta_{\alpha}^{2}\right]\left\langle\psi_{i}, \psi_{i}\right\rangle \\
& -\sum_{i=1}^{n} \sum_{j=1}^{n}\left[p_{3 i} p_{3 j} e_{\alpha i} e_{\alpha j}\right]<\psi_{i}, \psi_{j}><\psi_{i}, \psi_{j}> \\
& \left.-\delta_{\alpha \alpha} \sum_{i=1}^{n}\left[p_{3 i} e_{p \cdot y i}+p_{2 i} 2\right]<\psi_{i}, \psi_{i}\right\rangle \\
& -\frac{1}{r+E\left[e_{p \cdot y}^{2}\right]} \sum_{i=1}^{n}\left[p_{3 i} 4 e_{\alpha i} \hat{e}_{\alpha i} e_{p \cdot y i}\right] \\
& +4 \delta_{\alpha}^{2}\left(\frac{r}{r+E\left[e_{p \cdot y}^{2}\right]}\right)\left(1-\frac{r}{r+E\left[e_{p \cdot y}^{2}\right]}\right) \text {. }
\end{aligned}
$$

Since we absorbed the Jacobian into $L$, both $\frac{\partial w}{\partial \alpha}$ and $\frac{\partial^{2} w}{\partial \alpha^{2}}$ of (2.17), (2.18) remain identically equal to zero, even though our prior is not flat in the new parameterisation.

\section{Examples}

In deriving our approximations, we wanted them to be both accurate and second order correct. The distinction between correctness and accuracy is drawn because merely showing that an approximation is second order correct does not guarantee finite sample performance, an issue that is best dealt with empirically. To establish correctness, we examine Normal regression models with no censoring and Gumbel location-scale models with censoring. For the former, the constrained mode of the Laplace posterior can be derived in closed form, under a flat prior, thus allowing us to perform the expansions directly in terms of the parameter of interest. Having established that the two separate approximations are in agreement, we then use numerical integration and well-known approximations to the noncentral- $t$ distribution and that of Fisher's- $z$ statistic to evaluate the finite sample accuracy of our suggestions. We modify the expressions in Johnson \& Kotz (1970), so as to separate the effect of increasing the degrees of freedom into a part due to sample size 
and one dependent on the number of parameters in our model. We show that focusing on the degrees of freedom alone can be seriously misleading in situations where the number of parameters increases with sample size.

We then repeat the process with a censored Gumbel data set from the literature, where the dependence of our results on the sample in hand does not allow us to come to general conclusions, but does reassure us that the applicability of our results extends beyond the normal model.

Before proceeding though, we shall place both the Normal and the Gumbel distributions within the context of the generalised log-Gamma family of error distributions, derive the derivatives of (3.4) up to fourth order and comment on the significance of cubic and quartic terms in our expansions as we move between members of this family.

A random variable $Z$ is said to have a standardised log-gamma distribution with parameter $s>0$ if $Z \stackrel{d}{=} \sqrt{s} \log (X / s)$, where $X \sim \operatorname{Gamma}(s, 1)$. It follows that its density is given by:

$$
f_{Z}(z ; s)=\frac{1}{\Gamma(s)} s^{s-\frac{1}{2}} \exp [\sqrt{s} z-s \exp (z / \sqrt{s}], \quad 0<s<\infty
$$

For $s=1$, the distribution of $Z$ reduces to that of the logarithm of a standard exponential random variable, also known as the standard Gumbel or extreme value distribution. 7 Further, it is shown in Lawless (1982) that $\lim _{k \rightarrow \infty} f_{Z}(z ; k)=\phi(z)$, the standard normal density. In order to facilitate programming, we express our results in terms of $X=s \exp (Z / \sqrt{s})$, for which the p.d.f, c.d.f and hazard function are readily obtainable from most computer packages.

For the observed cases in our sample, the individual loglikelihood contribution and its derivatives are given by: $g_{Z}(z)=x(z)-s^{\frac{1}{2}} z, g_{Z}^{\prime}(z)=x(z) s^{-\frac{1}{2}}-s^{\frac{1}{2}}, g_{Z}^{\prime \prime}(z)=x(z) s^{-1}$, $g_{Z}^{\prime \prime \prime}(z)=x(z) s^{-\frac{3}{2}}, g_{Z}^{\prime v}(z)=x(z) s^{-2}$. If no censoring has taken place, it follows that the weights defined in (3.8) satisfy: $1>p_{2 i}=\sqrt{s} p_{3 i}=s p_{4 i}=\exp \sqrt{s} \hat{z}_{i} / \sum_{j=1}^{n} \exp \sqrt{s} \hat{z}_{j}>0$. We can then think of $\left\{p_{2 i}\right\}_{i=1}^{n}$ as a bona-fide probability measure, while $\left.\left\{p_{3 i}\right\}_{i=1}^{n}, p_{4 i}\right\}_{i=1}^{n}$ can be taken as positive finite measures with masses $1 / \sqrt{s}, 1 / s$ respectively. So, within the $\log$-gamma family, the importance of the third and fourth derivatives of $g$ in our expansions decreases smoothly as we move from the Gumbel to the normal. The opposite is true as $k$ decreases from 1 to 0 .

For censored cases, we have that $g_{Z}(z)=-\log \left[1-F_{X}(x(z))\right]$, where $F_{X}(x)$ is the well-known $\operatorname{Gamma}(s, 1)$ distribution. Consequently, setting $h_{X}(x)=f_{X}(x) / 1-F_{X}(x)$, $q_{X}(x)=\log f_{X}(x)$ and using the chain rule, allows us to express the derivatives of $g_{Z}(z)$

\footnotetext{
7The importance of the Gumbel as a location-scale density arises from its connection to the Weibull . Namely, if $\exp \left(\frac{Y-\mu}{\sigma}\right) \stackrel{d}{=}(a T)^{b} \stackrel{d}{=} S \sim$ exponential (1)for some $\mu=-\log a, \sigma=b^{-1}$, then, by definition, $Y \sim \operatorname{Gumbel}(\mu, \sigma)$, and $T \sim$ Weibull $(a, b)$. The hazard of $T=e^{\mu} S^{\sigma}$ is given by $h_{T}(t)=(a b)(a t)^{b-1}$. It is increasing for $b>1$, constant for $b=1$ and decreasing for $b<1$. Flexible enough to handle a lot of real world situations, it has found widespread use in reliability analysis and popularised the Gumbel as the resulting loglifetime distribution.
} 
w.r.t. $z$ in terms of the derivatives of $h_{X}(x)$ w.r.t. $x$, evaluated at $x(z)$. Suppressing obvious subscripts we obtain:

$$
\begin{aligned}
& g^{\prime}=h x^{\prime} \\
& g^{\prime \prime}=h^{\prime}\left(x^{\prime}\right)^{2}+h x^{\prime \prime} \\
& g^{\prime \prime \prime}=h^{\prime \prime}\left(x^{\prime}\right)^{3}+3 h^{\prime} x^{\prime} x^{\prime \prime}+h x^{\prime \prime \prime} \\
& g^{\prime v}=h^{\prime \prime \prime}\left(x^{\prime}\right)^{4}+6 h^{\prime \prime}\left(x^{\prime}\right)^{2} x^{\prime \prime}+h^{\prime}\left(4 x^{\prime} x^{\prime \prime \prime}+3\left(x^{\prime \prime}\right)^{2}\right)+h x^{\prime v}
\end{aligned}
$$

Using $\frac{\partial^{m} x(z)}{\partial z^{m}}=x(z) s^{-\frac{m}{2}}$ this simplifies to:

$$
\begin{aligned}
& g^{\prime}=h x s^{-\frac{1}{2}} \\
& g^{\prime \prime}=\left[h^{\prime} x+h\right] x s^{-1} \\
& g^{\prime \prime \prime}=\left[h^{\prime \prime} x^{2}+3 h^{\prime} x+h\right] x s^{-\frac{3}{2}} \\
& g^{\prime v}=\left[h^{\prime \prime \prime} x^{3}+6 h^{\prime \prime} x^{2}+7 h^{\prime} x+h\right] x s^{-2} .
\end{aligned}
$$

The derivatives of $h_{X}(x)$ w.r.t. $x$ may themselves be expressed recursively in terms of the corresponding derivatives of $q_{X}(x)$ :

$$
\begin{aligned}
& h^{\prime}=h\left(h+q^{\prime}\right) \\
& h^{\prime \prime}=h^{\prime}\left(h+q^{\prime}\right)+h\left(h^{\prime}+q^{\prime \prime}\right), \\
& h^{\prime \prime \prime}=h^{\prime \prime}\left(h+q^{\prime}\right)+2 h^{\prime}\left(h^{\prime}+q^{\prime \prime}\right)+h\left(h^{\prime \prime}+q^{\prime \prime \prime}\right) .
\end{aligned}
$$

They can be easily coded, since the hazard $h_{X}(x)=x^{s-1} \exp (-x) / \int_{x}^{\infty} y^{s-1} \exp (-y) d y$ is readily available from statistical packages, while $q_{X}(x)=c-x+(s-1) \log x$ is of simple form. A remarkable simplification occurs for the standard Gumbel distribution, the above results implying that $p_{2 i}=p_{3 i}=p_{4 i}=\exp \hat{z}_{i} / \sum_{j=1}^{n} \exp \hat{z}_{j}$ whether the $i$ 'th observation was censored or not; the quadratic, cubic and quartic terms of our expansions are seen to be of equal importance in this case.

The limiting normal case needs to be handled separately. With $h_{Z}(z)=\phi(z) /(1-\Phi(z))$, the derivatives of $g$ are given by:

$$
\begin{aligned}
& g^{\prime}(z)=h(z) \\
& g^{\prime \prime}(z)=h^{\prime}(z)=[h(z)-z] h(z), \\
& g^{\prime \prime \prime}(z)=h^{\prime \prime}(z)=\left[2 h^{2}(z)-3 z h(z)+z^{2}-1\right] h(z), \\
& g^{\prime v}(z)=h^{\prime \prime \prime}(z)=\left[6 h^{3}(z)-12 z h^{2}(z)+\left(7 z^{2}-4\right) h(z)-\left(z^{3}-3 z\right)\right] h(z),
\end{aligned}
$$

and are straightforward to program.

\subsection{Normal Regression Models}

First we consider a sample $u_{1}, \ldots, u_{n}$ from a normal regression model with no censoring, where the covariate vectors have been centered to start with. We examine the marginals of the logscale parameter $\theta_{2}$, of the $p^{\prime}$ th quantile for observations with covariate vector equal to $E[w]=0, u_{p}(\theta)=\theta_{0}+z_{p} \exp \left(\theta_{2}\right)$, and of a realised error from the model $z_{t}(\theta)=$ 
$\left(u_{t}-\theta_{0}\right) \exp \left(-\theta_{2}\right)$. Under a flat prior for $\theta$ and

$$
\hat{\theta}_{0}=\sum_{i=1}^{n} u_{i} / n, \hat{\theta}_{1}=\left(W^{T} W\right)^{-1} W^{T} u, \hat{u}_{i}=\hat{\theta}_{0}+w_{i}^{T} \hat{\theta}_{1}, \quad \exp \left(2 \hat{\theta}_{2}\right)=\sum_{i=1}^{n}\left(u_{i}-\hat{u}_{i}\right)^{2} / n,
$$

the logposterior of $\omega^{T}=\left(\alpha, \beta^{T}, \delta\right)$ reduces to

$$
L(\omega)=c-n \delta-\frac{n}{2}\left(z_{p}^{2}-2 z_{p}\left(z_{p}+\alpha\right) e^{-\delta}+\left[1+\left(\alpha+z_{p}\right)^{2}+\beta^{T} \Sigma_{Y Y} \beta\right] e^{-2 \delta}\right),
$$

where $\alpha=\left(u_{p}(\theta)-u_{p}(\hat{\theta})\right) \exp \left(-\hat{\theta}_{2}\right)$ is the standardised $p$ 'th quantile, $\beta=\left(\theta_{1}-\hat{\theta}_{1}\right) \exp \left(-\hat{\theta}_{2}\right)$ is the vector of standardised regression slopes and $\delta=\theta_{2}-\hat{\theta}_{2}$ is the centered logscale parameter. The Hessian then takes the simple form

$$
-\frac{\partial^{2} L(\hat{\omega})}{\partial \omega \partial \omega^{T}}=n\left[\begin{array}{ccc}
1 & 0 & -z_{p} \\
0 & \Sigma_{Y Y} & 0 \\
-z_{p} & 0 & 2+z_{p}^{2}
\end{array}\right]
$$

The scale parameter

The derivatives required for the expansion around $\hat{\delta}=0$ of the Laplace approximation to the posterior of $\delta$ are given by

$$
\frac{\partial^{2} R}{\partial \delta^{2}}=-2 n, \frac{\partial^{3} R}{\partial \delta^{3}}=4 n, \frac{\partial^{4} R}{\partial \delta^{4}}=-8 n, \frac{\partial h}{\partial \delta}=-2 k, \frac{\partial^{2} h}{\partial \delta^{2}}=0,
$$

in agreement with (2.21)-(2.25). The density of $V_{\delta}=\delta\left(I^{\delta \delta}\right)^{-\frac{1}{2}}=\sqrt{2 n} \log (\sigma / \hat{\sigma}) \stackrel{d}{=} \sqrt{\frac{n}{2}} \log \left(\frac{n}{X_{n-k}^{2}}\right)$ can then be approximated by (2.1) with constants satisfying

$$
d_{1}=k / \sqrt{2 n}, \quad d_{2}=0, \quad F_{3}=\sqrt{2 / n}, \quad F_{4}=-2 / n .
$$

Since $F_{3}>0, F_{4}<0$, the profile likelihood has positive skew and thin tails. The Laplace approximation shifts the profile likelihood to the right $\left(d_{1}>0\right)$, i.e. introduces a simple bias correction and flattens it $\left(d_{1} F_{3}>0\right)$, to reflect the uncertainty about the unknown regression coefficients. The only change brought about by an increase in the number of regressors is in the constant $d_{1}$, which is a crude measure of the distance between the profile mode of $V_{\delta}$ and its marginal posterior mode. If we were to fit a $\log F_{m_{0}, n_{0}}\left(\mu_{0}, \sigma_{0}\right)$ density first to the profile posterior of $V_{\delta}$ and then to its Laplace posterior, we would find that

$$
m_{0}^{-1}=0, \quad n_{0}=n-l, \quad \sigma_{0}=\sqrt{n / 2}, \quad \mu_{0}=-\sqrt{n / 2} \log (1-l / n),
$$

with $l=0, k$ respectively. The latter is exact ${ }^{8}$ and has its mode at $\mu_{0} \approx k / \sqrt{2 n}=d_{1}$, whereas the former is maximised at 0 and does not account for the loss of $k$ degrees of freedom.

\footnotetext{
${ }^{8}$ We adopt the convention $0 / 0=0$.
} 
The accuracy of our expansions can be checked by expressing the Laplace posterior as a function of $\delta$ alone and differentiating it directly. Assuming that $\Sigma_{Y Y}$ is of full rank, the first derivative conditions at the constrained mode of $L$,

$$
\frac{\partial L(\hat{\omega}(\delta))}{\partial \alpha}=-n \hat{\alpha}(\delta) e^{-2 \delta}=0, \frac{\partial L(\hat{\omega}(\delta))}{\partial \beta}=-n \Sigma_{Y Y} \hat{\beta}(\delta) e^{-2 \delta}=0
$$

imply that $\hat{\alpha}(\delta), \hat{\beta}(\delta)=0$ and reduce the logprofile likelihood, $R(\delta)$, to the kernel of a $\frac{1}{2} \log \frac{n}{\mathcal{X}_{n}^{2}}$ density, irrespective of $k$. Since

$$
R(\delta)=-\frac{n}{2}\left[e^{-2 \delta}-(1-2 \delta)\right] \approx-n\left(2 \frac{\delta^{2}}{2 !}-4 \frac{\delta^{3}}{3 !}+8 \frac{\delta^{4}}{4 !}\right),
$$

our quartic approximation to $R$ is second order correct but not exact.

The log determinant ratio term of the Laplace posterior is linear in $\delta$ and our quadratic approximation to it is actually exact

$$
-\frac{1}{2} h(\delta)=-\frac{1}{2} \log \left|\left(\Sigma_{X X} e^{-2 \hat{\delta}}\right)^{-1}\left(\Sigma_{X X} e^{-2 \delta}\right)\right|=k \delta \text {. }
$$

By modifying the profile likelihood, via the determinant term, the Laplace posterior adjusts for the loss of $k$ degrees of freedom and recovers the true density of $\delta$, given by:

$$
\pi(\delta) \propto \exp \left[R(\delta)-\frac{1}{2} h(\delta)\right]=\exp \left[-(n-k) \delta-\frac{n}{2}\left(e^{-2 \delta}-1\right)\right] .
$$

Though asymptotically equivalent to the Laplace posterior, our second order approximation to it is not exact, but allows obtaining the true density of $\delta$ under the extra assumption that its tail behaviour is that of a $\log F$ density.

This example seems a little contrived, since in practice neither the Laplace posterior will be exact nor will our fitting of densities by derivative matching be as successful. So we might ask how good are the other approximations we developed for tail probabilities and quantiles. In this context, accuracy to second order is all we can expect and we do indeed get it. Since

$$
V_{\delta} \stackrel{d}{=} \sqrt{\frac{n}{2}} \log \left(\frac{n}{\mathcal{X}_{n-k}^{2}}\right) \Leftrightarrow \frac{V_{\delta}}{\sqrt{2 n}}+\frac{1}{2} \log \left(\frac{n-k}{n}\right) \stackrel{d}{=} \frac{1}{2} \log F_{(+\infty, n-k)},
$$

and Fisher's $Z_{\left(\nu_{1}, \nu_{2}\right)}^{F}$ has a $(1 / 2) \log F_{\left(\nu_{1}, \nu_{2}\right)}$ distribution, we can use the approximations provided in Johnson \& $\operatorname{Kotz}\left(1970\right.$, sec. 26.4) with $1 / \nu_{1}=0$ to check the accuracy of our own. The expansions they provide are solely in terms of the degrees of freedom $\nu_{2}=n-k$ and fail to separate the effects of increasing the number of parameters $k$ as well as the sample size $n$. Since this latter situation is of most interest to us, their expressions need to be modified to suit our purposes.

Using Fisher's suggestion to replace $\nu_{2}^{-1}$ by $\left(\nu_{2}-1\right)^{-1}$ to improve accuracy, we obtain

$$
E\left[V_{\delta}\right] \approx \frac{k+1}{\sqrt{2 n}}, \quad \operatorname{Var}\left[V_{\delta}\right] \approx 1+\frac{k+1}{n},
$$


which agrees with the expressions given in (2.3). Also, setting $\sigma^{-2}=\delta^{-1}=2(n-k)$ in (16) of Johnson \& Kotz (1970, p.82), we can express the $\gamma^{\prime}$ th quantile of $V_{\delta}$ in terms of the corresponding normal quantile $z_{\gamma}$ via the cubic equation

$$
\begin{aligned}
v_{\delta, \gamma} & =\sqrt{\frac{n}{n-k}}\left[\frac{\left.z_{(+\infty, n-k ; \gamma)}^{F}\right]+\frac{k}{\sigma}}{\sqrt{2 n}},\right. \\
& \approx z_{\gamma}+\frac{3 k+2}{3 \sqrt{2 n}}+\frac{(9 k+5) z_{\gamma}}{18 n}+\frac{z_{\gamma}^{2}}{3 \sqrt{2 n}}+\frac{z_{\gamma}^{3}}{18 n} .
\end{aligned}
$$

This is indeed what the approximation we proposed in (2.10) reduces to and has the desirable property of being strictly increasing in $z_{\gamma}$ for all $n, k$. It shows clearly how an increase in the number of regressors both flattens the density of $V_{\delta}$ and shifts it to the right.

If we are interested in evaluating single tail probabilities we can use the mean and variance corrected signed square root of the likelihood ratio statistic as an approximate normalising transformation of $V_{\delta}$. By (2.6) we may take $r_{\delta} \stackrel{a p p}{\sim} N\left(\mu_{\delta}, \sigma_{\delta}^{2}\right)$ with

$$
r_{\delta} \approx v_{\delta}\left[1-\frac{v_{\delta}}{3 \sqrt{2 n}}+\frac{v_{\delta}^{2}}{18 n}\right], \quad \mu_{\delta} \approx \frac{3 k+2}{3 \sqrt{2 n}}, \quad \sigma_{\delta}^{2} \approx 1+\frac{3 k+1}{9 n}
$$

It is easy to check that $r_{\delta}$ is strictly increasing in $v_{\delta}$, since $\frac{d r_{\delta}}{d v_{\delta}}$ is a an upwards sloping quadratic equation with no real roots. Of course, since this is one of the relatively few cases where $R\left(v_{\delta}\right)$ is known exactly, we would expect the accuracy of our intervals to increase even further if we were to replace the expansion of $r_{\delta}$ given above by the exact result $r_{\delta}=\operatorname{sign}\left(v_{\delta}\right) \sqrt{-2 R\left(v_{\delta}\right)}=\operatorname{sign}\left(v_{\delta}\right) \sqrt{n\left(\exp \left(-v_{\delta} \sqrt{2 / n}\right)-1+v_{\delta} \sqrt{2 / n}\right)}$. The expressions for $\mu_{\delta}, \sigma_{\delta}$ reveal that treating $-2 R\left(v_{\delta}\right)$ as an approximate $\chi_{(1)}^{2}$ variable can be seriously misleading for large $k$. Also, since $r_{\delta}$ is nonlinear in $v_{\delta}$, the usual standard normal approximation to $V_{\delta}$ yields results even more inaccurate than those based on the uncorrected likelihood ratio.

An asymptotically equivalent normalising transformation of $V_{\delta}$ is given by:

$$
z_{\gamma} \approx v_{\delta, \gamma}\left[1-\frac{3 k+1}{18 n}-\frac{v_{\delta, \gamma}}{3 \sqrt{2 n}}+\frac{v_{\delta, \gamma}^{2}}{18 n}\right]-\frac{3 k+2}{3 \sqrt{2 n}},
$$

as suggested by (2.5), but the latter does not allow us to benefit from the fact that $r_{\delta}$ is here available exactly.

In fig. 1 we compare the true distribution of $V_{\delta}$ with approximations based on the exact likelihood ratio statistic and its second order expansion. Though our approximations were developed for a fixed number of regressors and increasing sample size, we tested the accuracy of our results when $k \sim O\left(n^{\frac{1}{2}}\right)$, expecting our target audience to want to test more complex hypotheses as additional data points become available. We find that the approximation based on the exact LR statistic dominates the true distribution stochastically, whereas an approximation based on the expansion of the LR statistic has too long a right tail. For a regression model with $k=5$ regressors and $n-k=20$ degrees of freedom both 

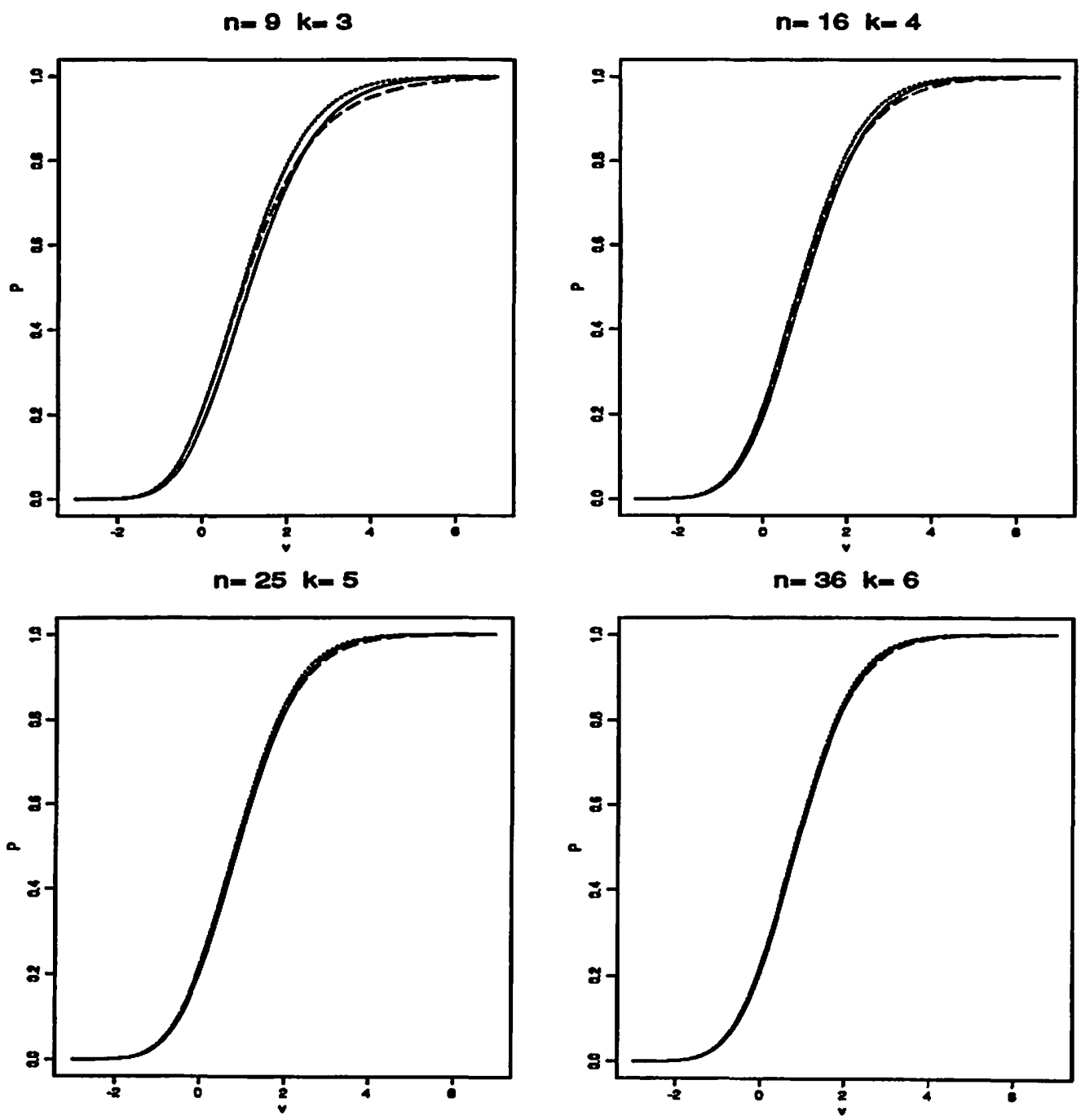

Figure 1: The cumulative distribution of $V_{\delta}$

True -, Exact LR - - , Approx LR - - - 
approximations are satisfactory in all but the extreme upper tail, where the exact LR approximation is preferable, as expected. If the degrees of freedom were kept fixed at the levels shown and $k$ alone allowed to increase, the quality of the approximation would worsen significantly, showing the sensitivity of our approximations to the number of regressors as well as the degrees of freedom.

Finally let us assume that we are interested in the expectation and variance of the standardised precision parameter $\frac{\hat{\sigma}^{2}}{\sigma^{2}}=\exp (-2 \delta) \stackrel{d}{=} \frac{\mathcal{X}_{n-k}^{2}}{n}$. We can use the approximation (2.2) to the cumulant generating function of $V_{\delta}$ to obtain

$$
\begin{array}{r}
E\left[\left(\frac{\hat{\sigma}}{\sigma}\right)^{2 r}\right]=M_{V_{\delta}}\left[-2 r\left(I^{\delta \delta}\right)^{\frac{1}{2}}\right]=M_{V_{\delta}}[-2 r / \sqrt{2 n}] \approx \\
\exp \left[(r-k-1) \frac{r}{n}+\left(k+1-\frac{2}{3} r\right)\left(\frac{r}{n}\right)^{2}+\frac{2 r}{3}\left(\frac{r}{n}\right)^{3}\right]
\end{array}
$$

which correctly implies that

$$
E\left[\frac{\hat{\sigma}^{2}}{\sigma^{2}}\right]=1-\frac{k}{n}+O\left(n^{-2}\right), \operatorname{Var}\left[\frac{\hat{\sigma}^{2}}{\sigma^{2}}\right]=\frac{2}{n}\left(1-\frac{k}{n}\right)+O\left(n^{-3}\right) .
$$

The above expressions also make clear that second order accuracy in approximating the m.g.f of the standardised variable $V_{\delta}$ implies increasing accuracy in approximating higher order moments of $\frac{\hat{\sigma}^{2}}{\sigma^{2}}$, which is the scale most meaningful to us.

\section{The location parameter}

Setting $z_{p}=0$ reduces $\alpha$ to $\frac{\theta_{0}-\hat{\theta}_{0}}{\hat{\sigma}}$, the location parameter for observations with covariate vector $w=0$. By standard results on normal regression models $\alpha$ is marginally distributed as a $t_{n-k}\left(0,(n-k)^{-1}\right)$ variable. The derivatives required for the expansion around $\hat{\alpha}=0$ of the Laplace approximation to its density simplify to

$$
\frac{\partial^{2} R}{\partial \alpha^{2}}=-n, \frac{\partial^{3} R}{\partial \alpha^{3}}=0, \frac{\partial^{4} R}{\partial \alpha^{4}}=6 n, \frac{\partial h}{\partial \alpha}=0, \frac{\partial^{2} h}{\partial \alpha^{2}}=-2(k-1) .
$$

which agrees with (2.21)-(2.25). Again, they may be checked by first expressing the Laplace posterior as a function of $\alpha$ alone and then differentiating it directly. The first derivatives of $L$ at the constrained posterior mode $\hat{\omega}(\alpha)$ are given by

$$
\begin{aligned}
& \frac{\partial L(\hat{\omega}(\alpha))}{\partial \beta}=-n \Sigma_{Y Y} \hat{\beta}(\alpha) e^{-2 \hat{\delta}(\alpha)}=0 \\
& \frac{\partial L(\hat{\omega}(\alpha))}{\partial \delta}=-n\left(1-\left[1+\alpha^{2}+\hat{\beta}^{T}(\alpha) \Sigma_{Y Y} \hat{\beta}(\alpha)\right] e^{-2 \hat{\delta}(\alpha)}\right)=0 .
\end{aligned}
$$

Assuming $\Sigma_{Y Y}$ is of full rank, they imply that $\hat{\beta}(\alpha)=0, \hat{\delta}(\alpha)=\frac{1}{2} \log \left[1+\alpha^{2}\right]$. The profile loglikelihood of $\alpha$ is then immediately recognisable as the kernel of a $t_{n-1}\left(0,(n-1)^{-1}\right)$ 
density, irrespective of $k$,

$$
R(\alpha)=L(\hat{\omega}(\alpha))-L(0)=-\frac{n}{2} \log \left[1+\alpha^{2}\right] .
$$

The log determinant ratio term can also be evaluated exactly,

$$
-\frac{1}{2} h(\alpha)=-\frac{1}{2} \log \left|\left(\begin{array}{cc}
\Sigma_{Y Y} & 0 \\
0 & 2
\end{array}\right)^{-1}\left(\begin{array}{cc}
\Sigma_{Y Y} e^{-2 \hat{\delta}(\alpha)} & 0 \\
0 & 2
\end{array}\right)\right|=\frac{k-1}{2} \log \left[1+\alpha^{2}\right],
$$

and implies that the Laplace posterior is the kernel of a $t_{n-k}\left(0,(n-k)^{-1}\right)$ density. It is, therefore, proportional to the exact density of $\alpha$, whose logarithm can be expanded as

$$
\begin{aligned}
\log \pi(\alpha) & =c+R(\alpha)-\frac{1}{2} h(\alpha)=c-\frac{n-k+1}{2} \log \left[1+\alpha^{2}\right] \\
& \simeq c-n\left(1-\frac{k-1}{n}\right)\left(\alpha^{2} / 2 !-6 \alpha^{4} / 4 !\right) .
\end{aligned}
$$

Fitting a $t$-density to the quartic approximation of $\log \pi(\alpha)$ above, by matching derivatives at the profile mode $\alpha=0$, also recovers the exact density of $\alpha$. Our second order approximation to $\log \pi(\alpha)$, however, uses only a quadratic expansion of $h(\alpha)$ and differs from (4.4) in that it drops the $O\left(n^{-2}\right)$ term given by $-(k-1) \alpha^{4} / 4$. As a result, it is more diffuse than the true density of $\alpha$ for $k>1$, the discrepancy getting larger with increasing $k$. For finite $n$, it is thus afflicted by a dimensionality problem that does not affect the asymptotically equivalent Laplace approximation. This time, knowledge of the tail behaviour of $\pi(\alpha)$ is of no help either. Since

$$
d_{1}=0, \quad d_{2}=(k-1) / n, \quad F_{3}=0, \quad F_{4}=6 / n,
$$

fitting a $t_{\nu}\left(0, \frac{\nu+1}{\nu} \frac{1}{1-d_{2}}\right)$ distribution with $\nu=n\left[1-d_{2}\right]^{2}-1$ degrees of freedom to the standardised variable $V_{\alpha}=\alpha\left(I^{\alpha \alpha}\right)^{-\frac{1}{2}} \stackrel{d}{=} \sqrt{n} \alpha=t_{n-k}(0, n /(n-k))$ also leads to incorrect results for $k>1$. It is easy to see that a quartic expansion of $h(\alpha)$ would remedy this problem, but it is very hard to obtain in general, though trivial in this particular case. This example then seems to suggest that the recommendations of Viveros \& Sprott (1987) for the location-scale model do not easily generalise to regression models.

Since expansions for $t$-densities are widely available, we can again check the accuracy of our formulae. For the central moments of $V_{\alpha} \stackrel{d}{=} \sqrt{\frac{n}{n-k}} T_{n-k}$ we adapt (5) of Johnson \& Kotz (1970, sec. 27.2) and find that

$$
\begin{aligned}
& \mu_{2}=\frac{n}{(n-k-2)} \approx 1+\frac{2+k}{n}, \\
& \mu_{4}=3\left(\frac{n}{n-k-2}\right)\left(\frac{n}{n-k-4}\right) \approx 3\left(\frac{1+2 k+6}{n}\right),
\end{aligned}
$$

with $\mu=\mu_{3}=0$ by symmetry, which agrees with the expressions in (2.2). Though correct to second order, our approximations to $\mu_{2}, \mu_{4}$ are actually seen to be underestimates for 
all $n, k$. It is worth noting that they were derived via an approximation to the moment generating function of $V_{\alpha}$, which in this case is infinite for all $n$ in any nontrivial neighbourhood of the origin. If this sounds surprising, it should be noted that Tierney et al. (1989) obtained a numerical approximation of $M_{V_{a}}(t)$ and motivated it by arguing that it could be viewed as an approximation to the m.g.f. of an appropriately truncated $t$-variable with moments differing from the exact $t$ moments by an error exponentially decreasing in the degrees of freedom.

For the quantiles of $V_{\alpha}$, we adapt (15) of Johnson \& Kotz (1970, sec. 27.4) and obtain

$$
v_{\alpha, \gamma}=\sqrt{\frac{n}{n-k}} t_{n-k, \gamma} \approx z_{\gamma}\left[1+\frac{1}{4 n}\left(1+2 k+z_{\gamma}^{2}\right)\right]
$$

which again agrees with the expression in (2.10) and is strictly increasing in $z_{\gamma}$. The table they provide shows that for $\gamma=.975$ our expression for $v_{\alpha, \gamma}$ has first digit accuracy for $n-k \geq 10$ and second digit accuracy for $n-k \geq 30$. If we examine their formula (16), we see that our approximation is actually third order correct but still consistently underestimates the true absolute value of $v_{\alpha, \gamma}$.

Tail probabilities can be evaluated by letting $r_{\alpha} \stackrel{a p p}{\sim} N\left(\mu_{\alpha}, \sigma_{\alpha}^{2}\right)$ where :

$$
r_{\alpha} \approx v_{\alpha}\left[1-\frac{v_{\alpha}^{2}}{4 n}\right], \quad \mu_{\alpha} \approx 0, \quad \sigma_{\alpha}^{2} \approx 1+\frac{1}{2 n}[2 k+1] .
$$

In this case $\frac{d r_{\alpha}}{d v_{\alpha}}$ is positive only for $3 v_{\alpha}^{2} \leq 4 n$, and our tail approximation is unreliable for small $n$. The exact signed square root of the likelihood ratio $r_{\alpha}=\operatorname{sign}\left(v_{\alpha}\right) \sqrt{-2 R\left(v_{\alpha}\right)}=$ $\operatorname{sign}\left(v_{\alpha}\right) \sqrt{n \log \left[1+\frac{v_{\alpha}^{2}}{n}\right]}$ does not suffer from monotonicity problems and leads to more accurate intervals.

Figure 2 shows that in regression models with $k=4$ regressors and as few as $n-k=12$ degrees of freedom both approximations are very satisfactory even in the extreme tails, but when the degrees of freedom are inadequate or the number of regressors too large, the expansion of the LR may lead to nonsensical results, due to monotonicity problems. The approximation based on the exact LR is more robust but has a slight tendency to underestimate the tails of $V_{\alpha}$.

By (2.5), an asymptotically equivalent normalising transformation of $V_{\alpha}$ is

$$
z_{\gamma} \approx v_{\alpha, \gamma}\left[1-\frac{1}{4 n}\left(v_{\alpha, \gamma}^{2}+2 k+1\right)\right] \text {. }
$$

It is monotone over the even narrower range $3 v_{\alpha}^{2} \leq 4 n-2 k-1$ and agrees with the first two terms of formula (14) of Johnson \& Kotz $(1970, \sec 27.4)$ on which their Table 4 is based. The row of that table corresponding to $x_{1}$ shows that the approximation possesses first digit accuracy even in the extreme tails for $n-k \geq 30$ but systematically underestimates $\left|z_{\gamma}\right|$, i.e. overestimates the tail probabilities. 

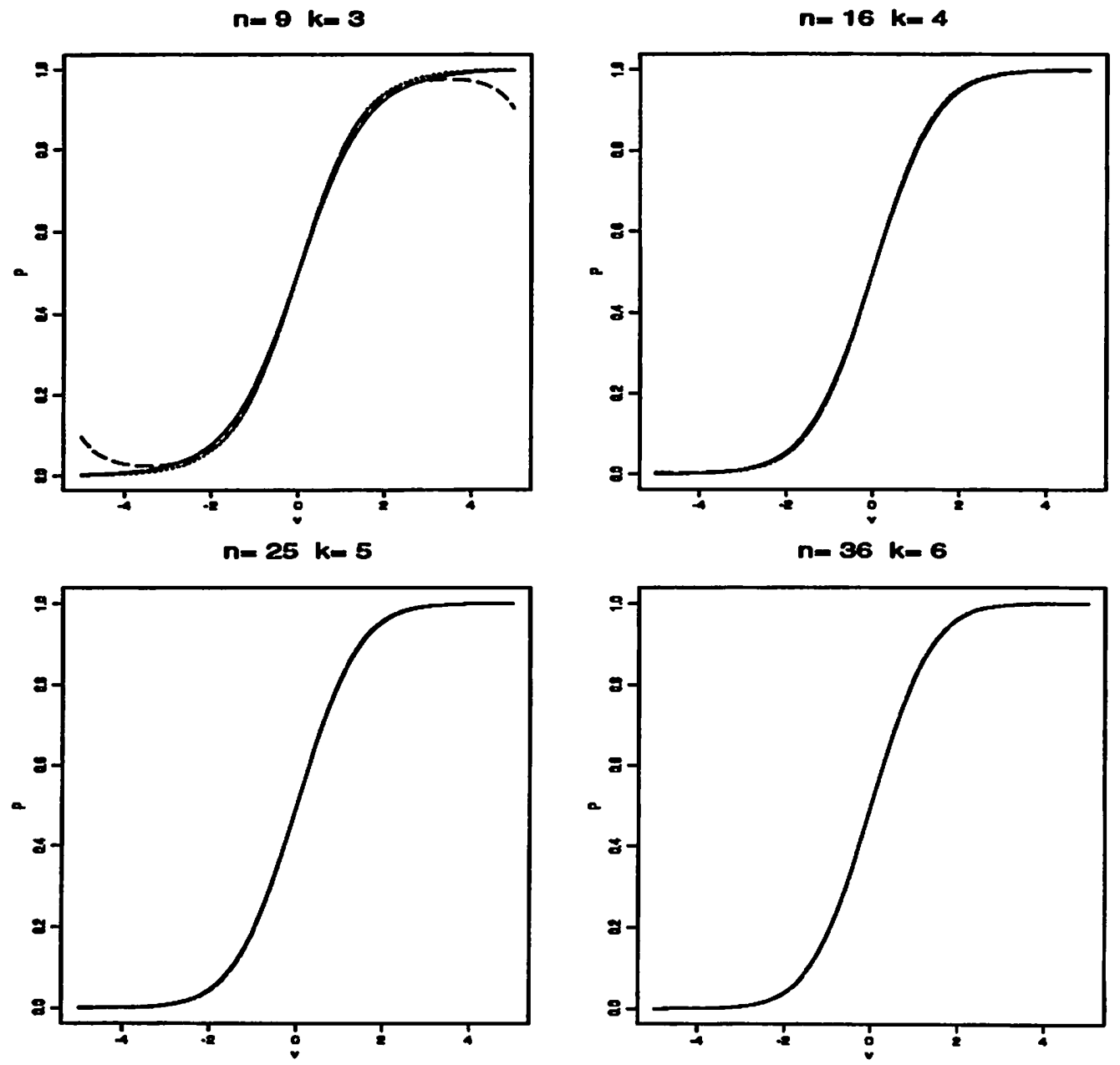

Figure 2: The cumulative distribution of $V_{\alpha}$ when $p=0.5$

True -, Exact LR - - , Approx LR --- 


\section{An arbitrary Quantile}

If we allow $\alpha$ to be an arbitrary quantile, not necessarily the one orthogonal to $\delta$, its distribution changes to that of a non-central $t_{n-k}\left(-z_{p},(n-k)^{-1} ; \sqrt{n} z_{p}\right)$ variable. The first derivatives at the constrained posterior mode $\hat{\omega}()$ simplify to:

$$
\begin{aligned}
& \frac{\partial L(\hat{\omega}(\alpha))}{\partial \beta}=-n \Sigma_{Y Y} \hat{\beta}(\alpha) e^{-2 \hat{\delta}(\alpha)}=0 \\
& \frac{\partial L(\hat{\omega}(\alpha))}{\partial \delta}=-n\left(1+z_{p}\left(z_{p}+\alpha\right) e^{-\hat{\delta}(\alpha)}-\left[1+\left(\alpha+z_{p}\right)^{2}\right] e^{-2 \hat{\delta}(\alpha)}\right)=0 .
\end{aligned}
$$

Assuming that $\Sigma_{Y Y}$ is full rank, we have that

$$
\hat{\beta}(\alpha)=0, \quad \exp \hat{\delta}(\alpha)=\frac{-z_{p}\left(\alpha+z_{p}\right)+\sqrt{4+\left(4+z_{p}^{2}\right)\left(\alpha+z_{p}\right)^{2}}}{2}
$$

The relative profile $\log$ posterior is then given by

$$
R(\alpha)=-n \hat{\delta}(\alpha)-\frac{n}{2}\left(1+z_{p}^{2}-\left[1+\left(\alpha+z_{p}\right)^{2}\right] e^{-2 \hat{\delta}(\alpha)}\right)
$$

while the log determinant ratio term reduces to

$$
-\frac{1}{2} h(\alpha)=(k-1) \hat{\delta}(\alpha)-\frac{1}{2} \log \left(\frac{1+\left[1+\left(\alpha+z_{p}\right)^{2}\right] e^{-2 \hat{\delta}(\alpha)}}{2+z_{p}^{2}}\right) .
$$

The derivatives of $\hat{\delta}(\alpha)$ w.r.t. $\alpha$ satisfy at $\hat{\alpha}=0$

$$
\begin{aligned}
\dot{\delta} & =\frac{z_{p}}{2+z_{p}^{2}}, \quad \ddot{\delta}=\frac{8-z_{p}^{4}}{\left(2+z_{p}^{2}\right)^{3}}, \quad \dddot{\delta}=-\frac{2 z_{p}}{\left(2+z_{p}^{2}\right)^{5}}\left(72+38 z_{p}^{2}+2 z_{p}^{4}-z_{p}^{6}\right), \\
\dddot{\delta} & =-\frac{6}{\left(2+z_{p}^{2}\right)^{7}}\left(128-352 z_{p}^{2}-368 z_{p}^{4}-106 z_{p}^{6}-6 z_{p}^{8}+z_{p}^{10}\right),
\end{aligned}
$$

so that

$$
\begin{aligned}
\frac{\partial h}{\partial \alpha} & =\frac{2 z_{p}}{2+z_{p}^{2}}\left[\frac{1}{2+z_{p}^{2}}-(k-1)\right] \\
\frac{\partial^{2} h}{\partial \alpha^{2}} & =\frac{-2}{\left(2+z_{p}^{2}\right)^{3}}\left[\frac{10 z_{p}^{2}+3 z_{p}^{4}}{2+z_{p}^{2}}+(k-1)\left(8-z_{p}^{4}\right)\right] \\
\frac{\partial^{2} R}{\partial \alpha^{2}} & =\frac{-2 n}{2+z_{p}^{2}}, \quad \frac{\partial^{3} R}{\partial \alpha^{3}}=\frac{2 n\left(12 z_{p}+5 z_{p}^{3}\right)}{\left(2+z_{p}^{2}\right)^{3}} \\
\frac{\partial^{4} R}{\partial \alpha^{4}} & =\frac{6 n\left(32-32 z_{p}^{2}-40 z_{p}^{4}-9 z_{p}^{6}\right)}{\left(2+z_{p}^{2}\right)^{5}}
\end{aligned}
$$


which is exactly what the derivatives in (2.21)-(2.25) simplify to. We have thus checked the second order correctness of our approximation to the posterior distribution of an arbitrary normal quantile. The question of accuracy remains, however, and the determinant term (4.6) suggests a possible dimensionality problem. By assuming that $\alpha \sim O\left(n^{-\frac{1}{2}}\right)$ and that the number of regressors is fixed, our second order expansion to $h(\alpha)$ replaces $\delta(\alpha)$ by a quadratic in $\alpha$. The first term omitted is then given by $\dddot{\delta}(k-1) \alpha^{3} / 24$. In practice both the sample size and the number of regressors are fixed, and, if for the particular sample in hand $k \sim O\left(n^{\frac{1}{2}}\right)$, the first term omitted will actually behave as $O\left(n^{-1}\right)$ and our approximation to $h(\alpha)$ will only be first order correct, unless $\dddot{\delta}$ is close to zero. The plot of $\dddot{\delta}$ versus $z_{p}$ in fig. 3 shows this to be the case for the extreme quantiles. Since, apart from the median, those tend to be the quantiles of most interest in reliability theory, our approximation remains useful even for a moderate number of regressors. For the other quantiles, however, our approximations will be severely afflicted by a dimensionality problem. An examination of the plot of $\dddot{\delta}$ versus $z_{p}$ in fig. 4 only serves to reinforce this conclusion.

From above, the constants needed for the approximation to the density of $V_{\alpha}=\sqrt{\frac{2 n}{2+z_{p}^{2}}} \alpha$ are given by:

$$
\begin{aligned}
& d_{1}=-\frac{1}{\sqrt{2 n}} \frac{z_{p}}{\left(2+z_{p}^{2}\right)^{\frac{1}{2}}}\left[\frac{1}{2+z_{p}^{2}}-(k-1)\right] \\
& d_{2}=+\frac{1}{2 n} \frac{1}{\left(2+z_{p}^{2}\right)^{2}}\left[\frac{10 z_{p}^{2}+3 z_{p}^{4}}{\left(2+z_{p}^{2}\right)}+(k-1)\left(8-z_{p}^{4}\right)\right], \\
& F_{3}=+\frac{1}{\sqrt{2 n}} \frac{12 z_{p}+5 z_{p}^{3}}{\left(2+z_{p}^{2}\right)^{\frac{3}{2}}}, \quad F_{4}=+\frac{3}{2 n} \frac{\left(32-32 z_{p}^{2}-40 z_{p}^{4}-9 z_{p}^{6}\right)}{\left(2+z_{p}^{2}\right)^{3}}
\end{aligned}
$$

and provide a tail probability approximation that can be checked against exact results available for the noncentral $t$ distribution. Since the density of the noncentral $t$ distribution is only available as a hard-to-analyse infinite series, the above constants also provide a nice summary of the changes in location, scale and shape of the marginal distribution of the $p$-th quantile, as $p$ varies over $(0,1)$. For $k=1$, they reduce to the results in DiCiccio (1987).

In fig. 5 we plot the standardised derivatives of the approximate density of $V_{\alpha}$, evaluated at $\hat{\alpha}=0$, versus $z_{p}$. We see that more extreme quantiles are characterised by increasing skewness and a reduction in tail weight, suggesting that one tail is brought in while the other is elongated. For location-scale models $(k=1)$, both $d_{1}$ and $d_{2}$ are essentially constant throughout and the profile posterior is by itself a satisfactory approximation to the true marginal. As the number of regressors increases for $n$ fixed, the substantial changes in $d_{1}, d_{2}$ suggest that a recentering and rescaling of the profile posterior is warranted.

In fig. 6 we examine the distribution of the standardised first percentile, which is seen to possess an extremely long left tail. When there are too many regressors relative to the degrees of freedom, our approximations tend to severely underestimate the left tail; the situation improves rapidly as more observations become available and seems satisfactory for $k=6$ regressors and $n-k=30$ degrees of freedom. 


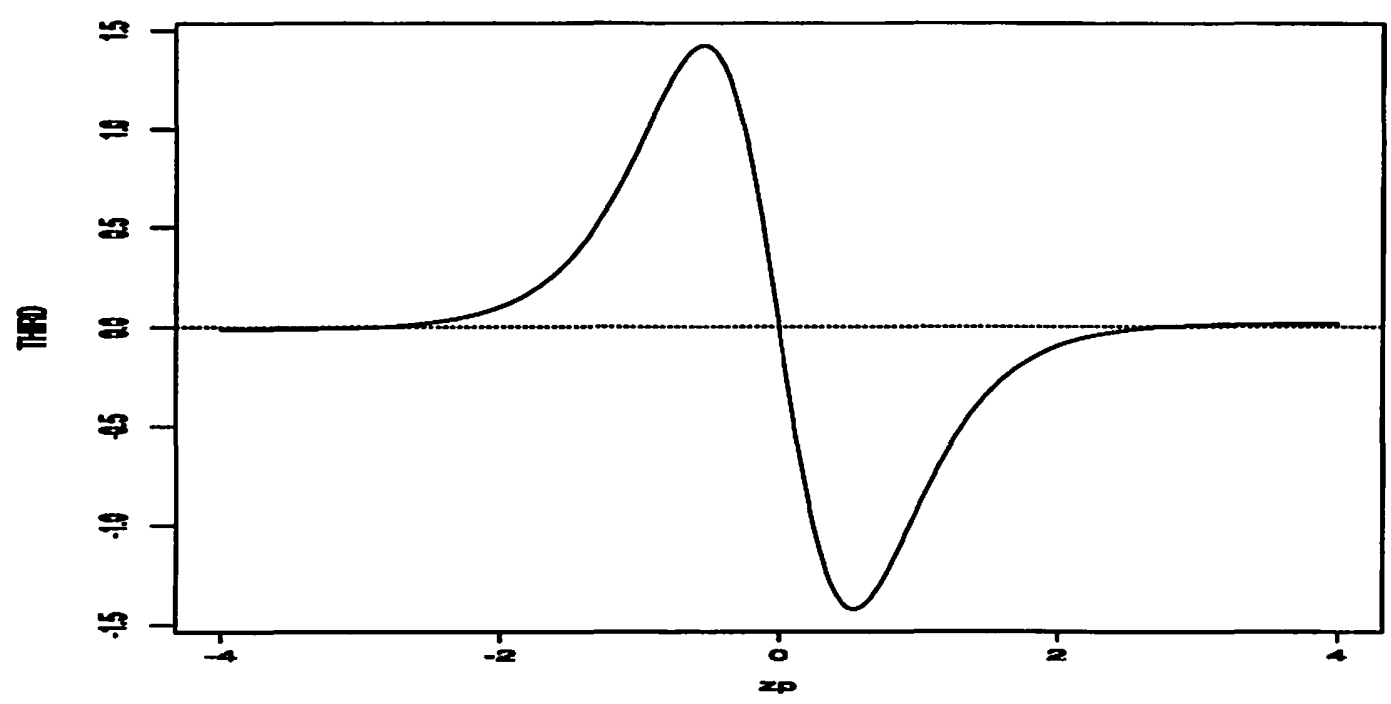

Figure 3: The third derivative of $\hat{\delta}(\alpha)$ at $\hat{\alpha}=0$ vs. $z_{p}$

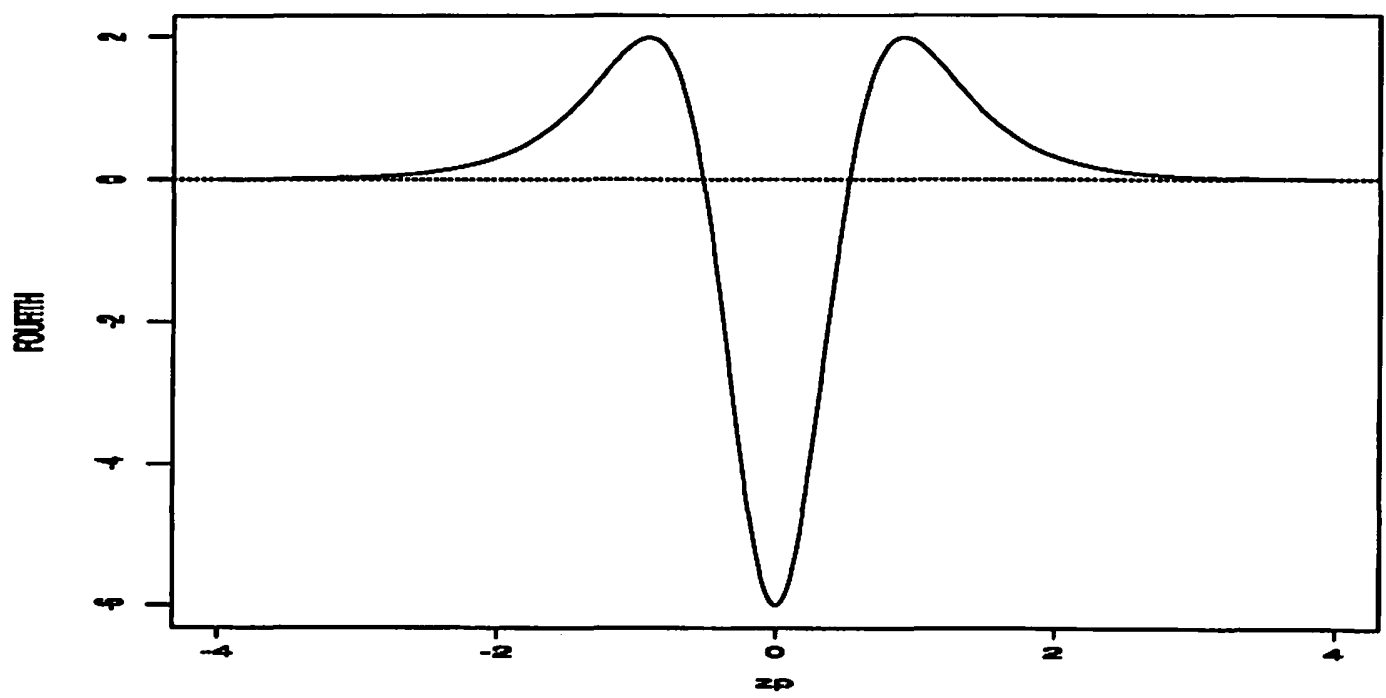

Figure 4: The fourth derivative of $\hat{\delta}(\alpha)$ at $\hat{\alpha}=0$ vs. $z_{p}$ 

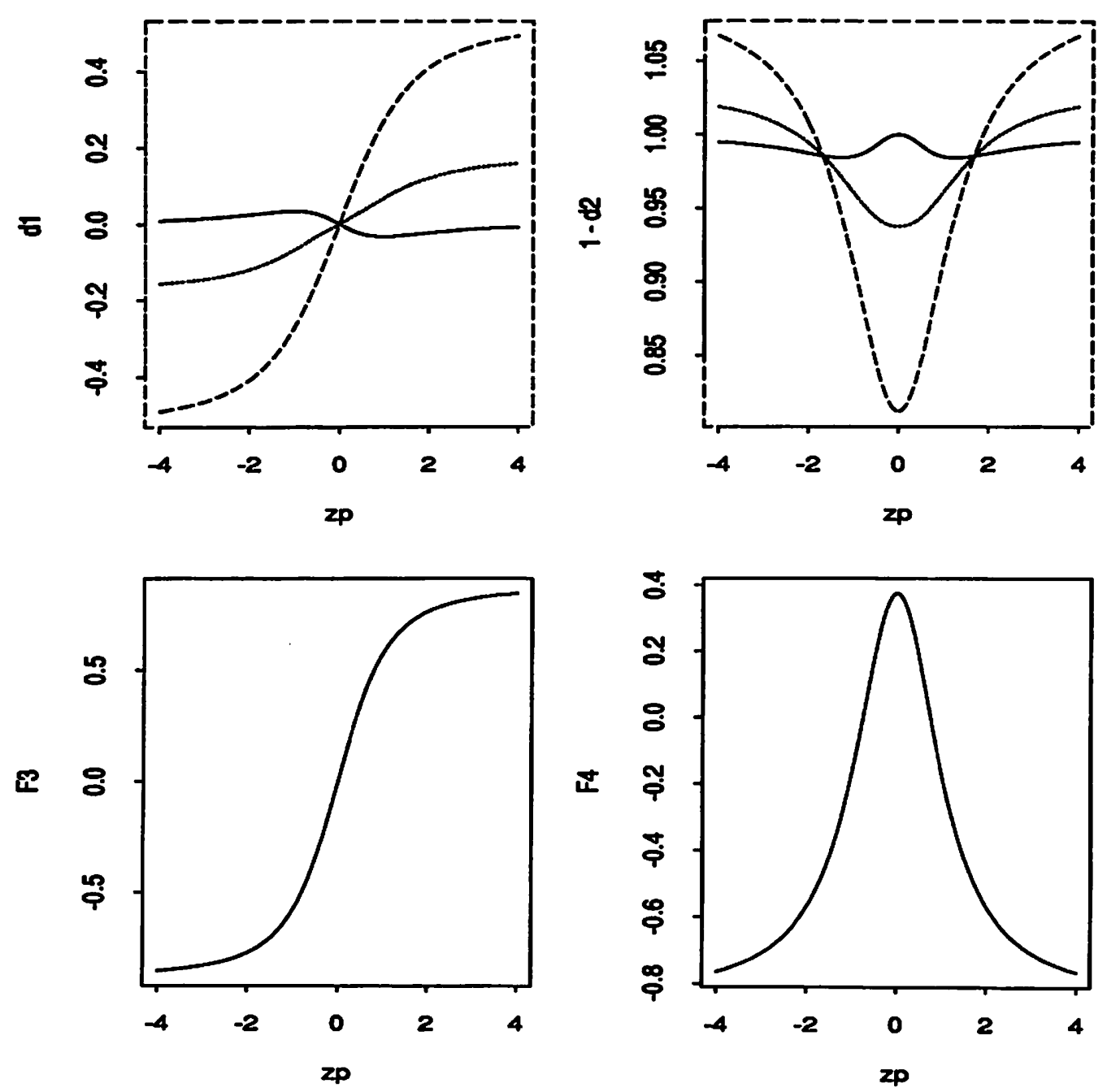

Figure 5: The derivatives of the approximate density of $V_{\alpha}$ at $\hat{\alpha}=0$

$$
n=16, k=1-, k=2--, k=4 \cdots
$$



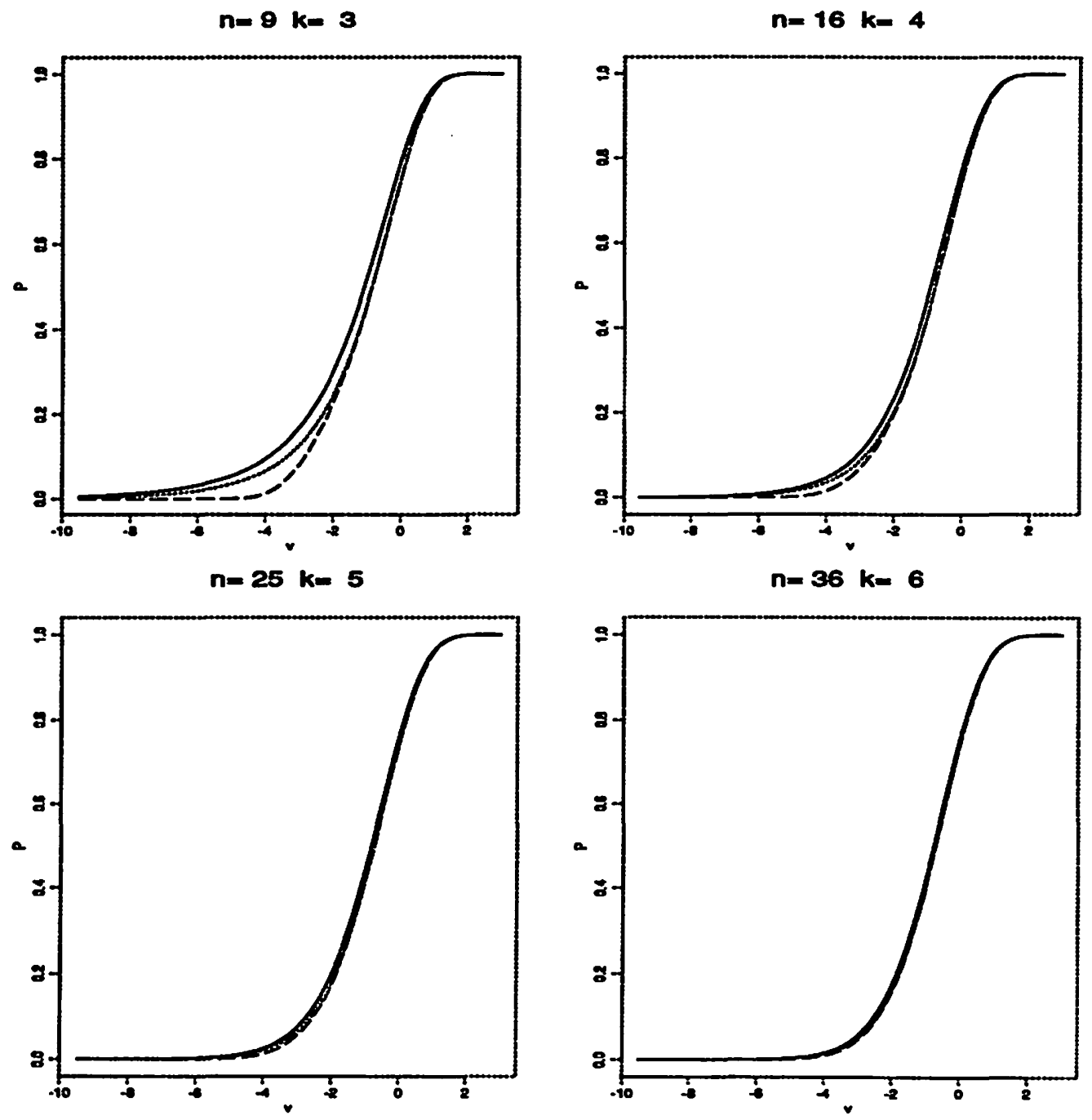

Figure 6: The cumulative distribution of $V_{\alpha}$ when $p=0.01$ True -, Exact LR - - , Approx LR - - 


\section{Survival probabilities and realised errors.}

We now suppose that interest centers on credible intervals for the survival probability beyond $u_{t}=\log t$ for a future observation whose covariate vector is $E[w]=0$. With $z_{t}(\theta)=\left(u_{t}-\theta_{0}\right) \exp \left(-\theta_{2}\right)$, this is given by $\Phi\left[\left(z_{t}(\theta)\right]\right.$, a strictly increasing function of $z_{t}(\theta)$ whose distribution we shall approximate instead. If $u_{t}$ is an observed loglifetime, $z_{t}$ can also be interpreted as a realised error from the model, expressed in standard units. If we adopt a flat prior for $\theta$ and set

$$
\hat{\theta}_{0}=\frac{\sum_{i=1}^{n} u_{i}}{n}, \hat{\theta}_{1}=\left(W^{T} W\right)^{-1} W^{T} u, \hat{u}_{i}=\hat{\theta}_{0}+w_{i}^{T} \hat{\theta}_{1}, \exp \left(2 \hat{\theta}_{2}\right)=\frac{\sum_{i=1}^{n}\left(u_{i}-\hat{u}_{i}\right)^{2}}{(n-1)},
$$

we can reparameterise in terms of $\alpha=z_{t}(\hat{\theta})-z_{t}(\theta), \beta=\left(\theta_{1}-\hat{\theta}_{1}\right) \exp \left(-\hat{\theta}_{2}\right), \delta=\theta_{2}-\hat{\theta}_{2}$. The logposterior of $\omega^{T}=\left(\alpha, \beta^{T}, \delta\right)$ is then given by

$$
L(\omega)=c-n r \delta-\frac{n}{2}\left[\left(\hat{z}_{t}-\alpha\right)^{2}-2 \hat{z}_{t}\left(\hat{z}_{t}-\alpha\right) e^{-\delta}+\left(r+\hat{z}_{t}^{2}+\beta^{T} \Sigma_{Y Y} \beta\right) e^{-2 \delta}\right]
$$

and the Hessian takes the form

$$
-\frac{\partial^{2} L(\hat{\omega})}{\partial \omega \partial \omega^{T}}=n\left[\begin{array}{ccc}
1 & 0 & -\hat{z}_{t} \\
0 & \Sigma_{Y Y} & 0 \\
-\hat{z}_{t} & 0 & 2 r+\hat{z}_{t}^{2}
\end{array}\right] .
$$

In moving from (4.1) to (4.7), we accommodated the nonconstant Jacobian by redefining $\hat{\theta}_{2}$. As a result, (4.7) is still maximised at zero. The connection with frequentist inference is, however, lost, since now $\alpha$ is no longer a pivotal quantity. The derivatives of (2.21)-(2.25) are then found to be equal to

$$
\begin{aligned}
\frac{\partial^{2} R}{\partial \alpha^{2}} & =\frac{-2 r n}{2 r+\hat{z}_{t}^{2}}, \quad \frac{\partial^{3} R}{\partial \alpha^{3}}=\frac{-2 r n \hat{z}_{t}^{3}}{\left(2 r+\hat{z}_{t}^{2}\right)^{3}}, \quad \frac{\partial^{4} R}{\partial \alpha^{4}}=\frac{-6 r n \hat{z}_{t}^{6}}{\left(2 r+\hat{z}_{t}^{2}\right)^{5}}, \\
\frac{\partial h}{\partial \alpha} & =\frac{-2 \hat{z}_{t}}{2 r+\hat{z}_{t}^{2}}\left[\frac{r+\hat{z}_{t}^{2}}{2 r+\hat{z}_{t}^{2}}+k-1\right], \\
\frac{\partial^{2} h}{\partial \alpha^{2}} & =\frac{-2 \hat{z}_{t}^{2}}{\left(2 r+\hat{z}_{t}^{2}\right)^{3}}\left[\frac{r+\hat{z}_{t}^{2}}{2 r+\hat{z}_{t}^{2}}\left(\hat{z}_{t}^{2}-2 r\right)+(k-1) \hat{z}_{t}^{2}\right] .
\end{aligned}
$$

and can be checked by expressing the Laplace posterior as a function of $\alpha$ alone. We first notice that the first derivatives at the constrained posterior mode simplify to

$$
\begin{aligned}
& \frac{\partial L(\hat{\omega}(\alpha))}{\partial \beta}=-n \Sigma_{Y Y} \hat{\beta}(\alpha) e^{-2 \hat{\delta}(\alpha)}=0 \\
& \frac{\partial L(\hat{\omega}(\alpha))}{\partial \delta}=-n\left[r+\hat{z}_{t}\left(\hat{z}_{t}-\alpha\right) e^{-\hat{\delta}(\alpha)}-\left(r+\hat{z}_{t}^{2}\right) e^{-2 \hat{\delta}(\alpha)}\right]=0 .
\end{aligned}
$$


It follows that the constrained posterior mode of $(\beta, \delta)$ satisfies

$$
\hat{\beta}(\alpha)=0, \quad \exp \hat{\delta}(\alpha)=\frac{-\hat{z}_{t}\left(\hat{z}_{t}-\alpha\right)+\sqrt{\hat{z}_{t}^{2}\left(\hat{z}_{t}-\alpha\right)^{2}+4 r\left(r+\hat{z}_{t}^{2}\right)}}{2 r} .
$$

The relative profile log posterior is then given by

$$
R(\alpha)=-n r \hat{\delta}(\alpha)-\frac{n}{2}\left[\alpha^{2}-2 \alpha \hat{z}_{t}+\left(r+\hat{z}_{t}^{2}\right)\left(1-e^{-2 \hat{\delta}(\alpha)}\right)\right],
$$

while the log determinant ratio term reduces to

$$
-\frac{1}{2} h(\alpha)=(k-1) \hat{\delta}(\alpha)-\frac{1}{2} \log \left[\frac{r+\left(r+\hat{z}_{t}^{2}\right) e^{-2 \hat{\delta}(\alpha)}}{2 r+\hat{z}_{t}^{2}}\right] .
$$

Tedious but straightforward calculations then show that the derivatives of $R, h$ are of the form claimed earlier. They involve the derivatives of $\hat{\delta}(\alpha)$, which evaluate at $\hat{\alpha}=0$ to

$$
\begin{aligned}
\dot{\delta} & =\frac{\hat{z}_{t}}{2 r+\hat{z}_{t}^{2}}, \quad \ddot{\delta}=\frac{\hat{z}_{t}^{4}}{\left(2 r+\hat{z}_{t}^{2}\right)^{3}}, \quad \dddot{\delta}=-\frac{2 \hat{z}_{t}^{3}}{\left(2 r+\hat{z}_{t}^{2}\right)^{5}}\left(2 r^{2}+2 r \hat{z}_{t}^{2}-\hat{z}_{t}^{4}\right), \\
\dddot{\delta} & =-\frac{6 \hat{z}_{t}^{6}}{\left(2 r+\hat{z}_{t}^{2}\right)^{7}}\left(6 r^{2}+6 r \hat{z}_{t}^{2}-\hat{z}_{t}^{4}\right) .
\end{aligned}
$$

We plot the last two versus $\hat{z}_{t}$ in figs. 7,8 when $n=16$. We see that both the cubic and quartic terms in the expansion of $\hat{\delta}(\alpha)$ are quite small for all values of $\hat{z}_{t}$. As $n$ increases, their shape remains the same but their magnitude is reduced even further. This in turn implies that, even though our quadratic approximation to $h(\alpha)$ obviously deteriorates as the number of regressors increases, the degradation should be quite small for moderate $k$.

The constants needed for the approximation to the density of $V_{\alpha}=\alpha\left(I^{\alpha \alpha}\right)^{-\frac{1}{2}}=$ $\sqrt{\frac{2 r n}{2 r+\hat{z}_{t}^{2}}}\left(\hat{z}_{t}-z_{t}\right)$ are given from above by:

$$
\begin{aligned}
& d_{1}=+\frac{1}{\sqrt{2 r n}} \frac{\hat{z}_{t}}{\left(2 r+\hat{z}_{t}^{2}\right)^{\frac{1}{2}}}\left[\frac{r+\hat{z}_{t}^{2}}{2 r+\hat{z}_{t}^{2}}+k-1\right], \\
& d_{2}=+\frac{1}{2 r n} \frac{\hat{z}_{t}^{2}}{\left(2 r+\hat{z}_{t}^{2}\right)^{2}}\left[\frac{r+\hat{z}_{t}^{2}}{2 r+\hat{z}_{t}^{2}}\left(\hat{z}_{t}^{2}-2 r\right)+(k-1) \hat{z}_{t}^{2}\right], \\
& F_{3}=-\frac{1}{\sqrt{2 r n}} \frac{\hat{z}_{t}^{3}}{\left(2 r+\hat{z}_{t}^{2}\right)^{\frac{3}{2}}}, \quad F_{4}=-\frac{3}{2 r n} \frac{\hat{z}_{t}^{6}}{\left(2 r+\hat{z}_{t}^{2}\right)^{3}},
\end{aligned}
$$

and are plotted versus $\hat{z}_{t}$ in fig 9 for $n=16$ and $k=1,2,4$. Increasing $n$ further would change the magnitude of the standardised derivatives, but not their shape. Of more interest is the effect of increasing $k$ for $n$ fixed. We see that this has no effect on $F_{3}, F_{4}$ and only a mild effect on $d_{2}$, but a very strong one on $d_{1}$. Thus, the Laplace posterior differs from the profile posterior mainly by a change in location, a shift which is accentuated as $\left|\hat{z}_{t}\right|$ increases. Since $3 F_{3}^{2}+F_{4}=0$, the kurtosis of the posterior is 0 to second order. This 


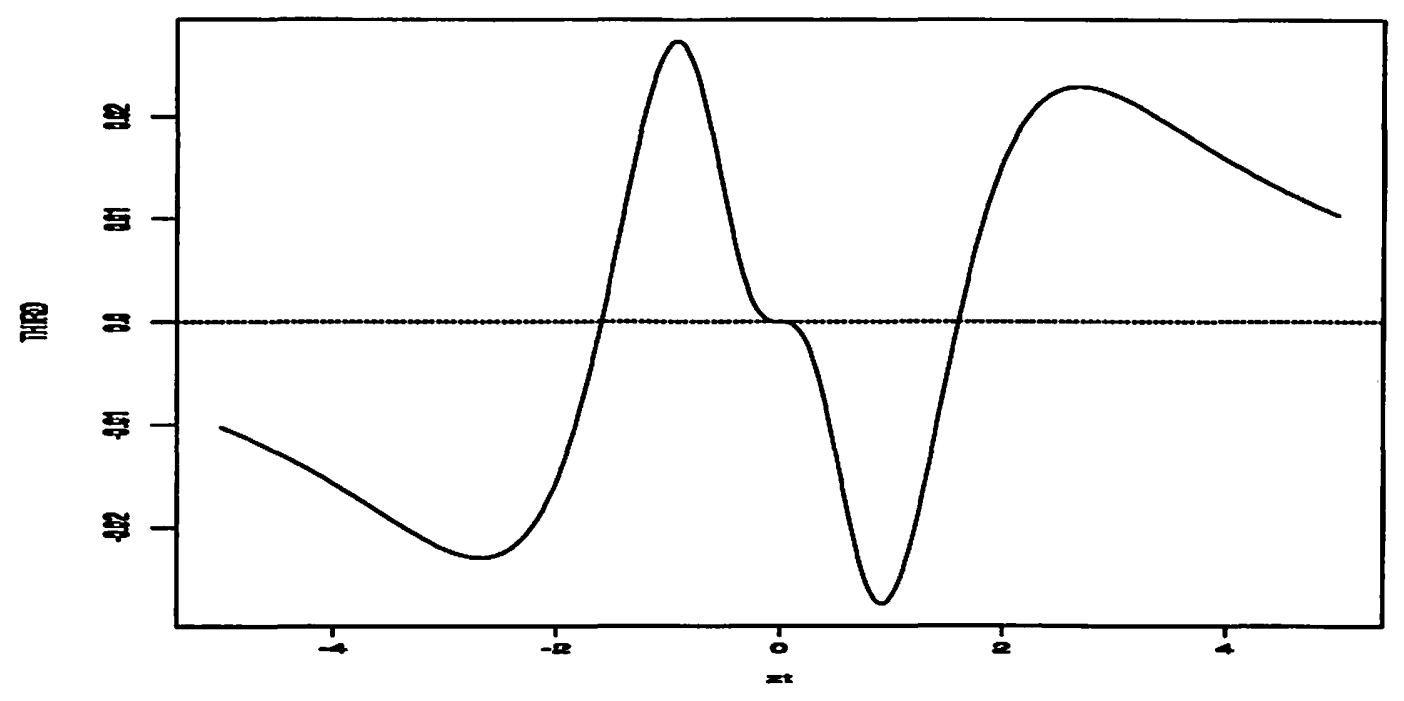

Figure 7: The third derivative of $\hat{\delta}(\alpha)$ at $\hat{\alpha}=0$ vs. $\hat{z}_{t}$ when $n=16$

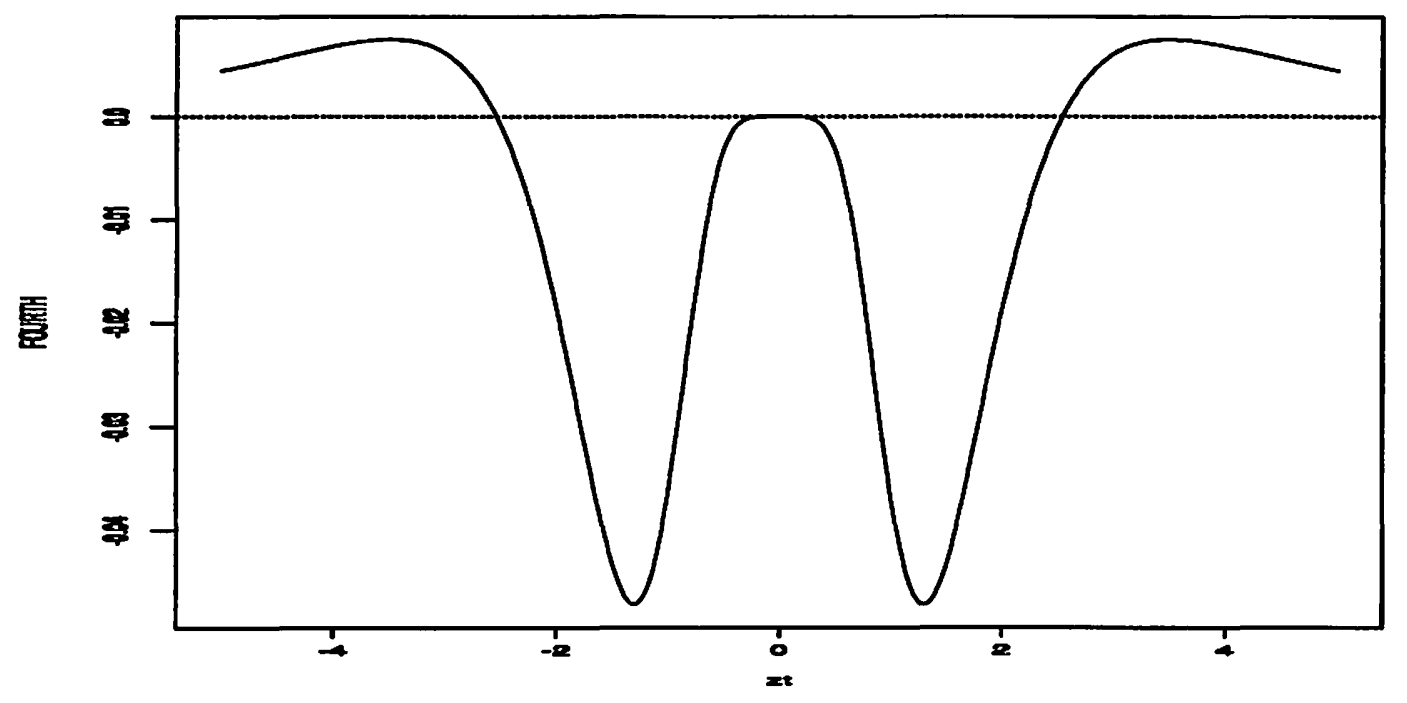

Figure 8: The fourth derivative of $\hat{\delta}(\alpha)$ at $\hat{\alpha}=0$ vs. $\hat{z}_{t}$ when $n=16$ 

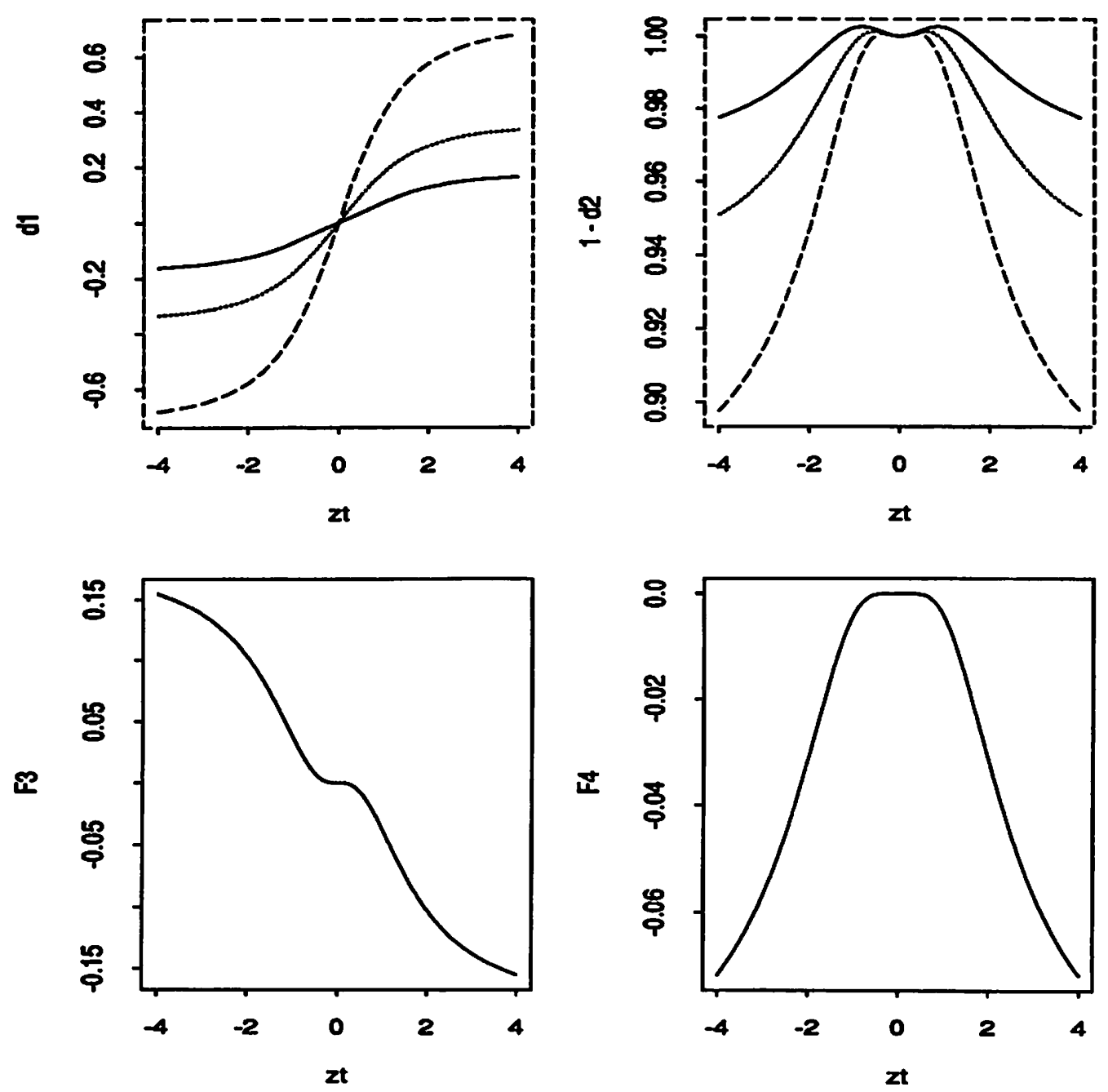

Figure 9: The derivatives of the approximate density of $V_{\alpha}$

$$
n=16, k=1-, k=2--, k=4-\cdots \text {. }
$$


also implies that $F_{3}^{2}+F_{4}<0$, so that the profile posterior is not approximable by a $\log F$ density, though the Laplace posterior might be.

Even though the marginal distribution of $\alpha$ is not available in closed form, we can show that its conditional distribution given $\delta$ is a $N\left(\hat{z}_{t}\left[1-e^{-\delta}\right], n^{-1}\right)$. Since $(n-1) e^{-2 \delta} \stackrel{d}{=} \chi_{n-k}^{2}$, we can then obtain exact credible intervals for $\alpha$ via a one-dimensional numerical integration. Our approximation above provides an alternative to numerical integration for $n-k$ sufficiently large.

In fig. 10 we plot the exact distribution function of $V_{\alpha}$ when $\hat{z}_{t}=4$, i.e. an outlying observation, and compare it to the LR approximations. Their accuracy seems impressive, even for problems with 3 regressors and 6 degrees of freedom! There is however a slight stochastic dominance of the exact distribution by our approximations, which would be exacerbated if we were to increase the number of regressors while keeping the degrees of freedom fixed. The c.d.f. plots create the misleading impression of a long right tail, which would seem surprising with $F_{3}<0$ at $\hat{z}_{t}=4$. What in fact happens is that $d_{1}$ dominates all other constants in the expansion, causing a pronounced rightward shift of the probability mass from $(-3,3)$ to $(-2,4)$ standard units, which is not compensated by the slight left skew.

\subsection{Gumbel Location-Scale Models}

For Gumbel errors the third and fourth derivatives of $g$ do not vanish, but they are equal to the second derivative and, hence, $p_{2 i}=p_{3 i}=p_{4 i}$. Also, $\sum_{i=1}^{n} g^{\prime \prime}\left(\hat{z}_{i}\right)$ equals $m$, the number of cases that were not censored, so that heavy censoring drastically reduces the sample information, on which our asymptotic approximations rely.

\section{The scale parameter}

The density of $V_{\delta}=\delta\left(I^{\delta \delta}\right)^{-\frac{1}{2}}$ is given by (2.1) with:

$$
\begin{aligned}
& d_{1}=(1 / \sqrt{m}) E\left[(1+\hat{p})^{2}\right]^{-\frac{1}{2}}, \quad d_{2}=0, \\
& F_{3}=(1 / \sqrt{m}) E\left[(1+\hat{p})^{3}\right] E\left[(1+\hat{p})^{2}\right]^{-\frac{3}{2}} \\
& F_{4}=-(1 / m)\left(E\left[(1+\hat{p})^{4}+(1+2 \hat{p}) \hat{p}^{2}\right]-3 E\left[\hat{p}^{2}\right]^{2}\right) E\left[(1+\hat{p})^{2}\right]^{-2} .
\end{aligned}
$$

In this case $F_{3}, F_{4}$ can be of either sign, but $d_{1}>0, d_{2}=0$, so that the determinant term is approximately linear and always shifts the profile likelihood to the right.

\section{The quantile orthogonal to the scale parameter}

In the special case when $\alpha$ is the quantile orthogonal to $\delta$ in the observed sense, the density 

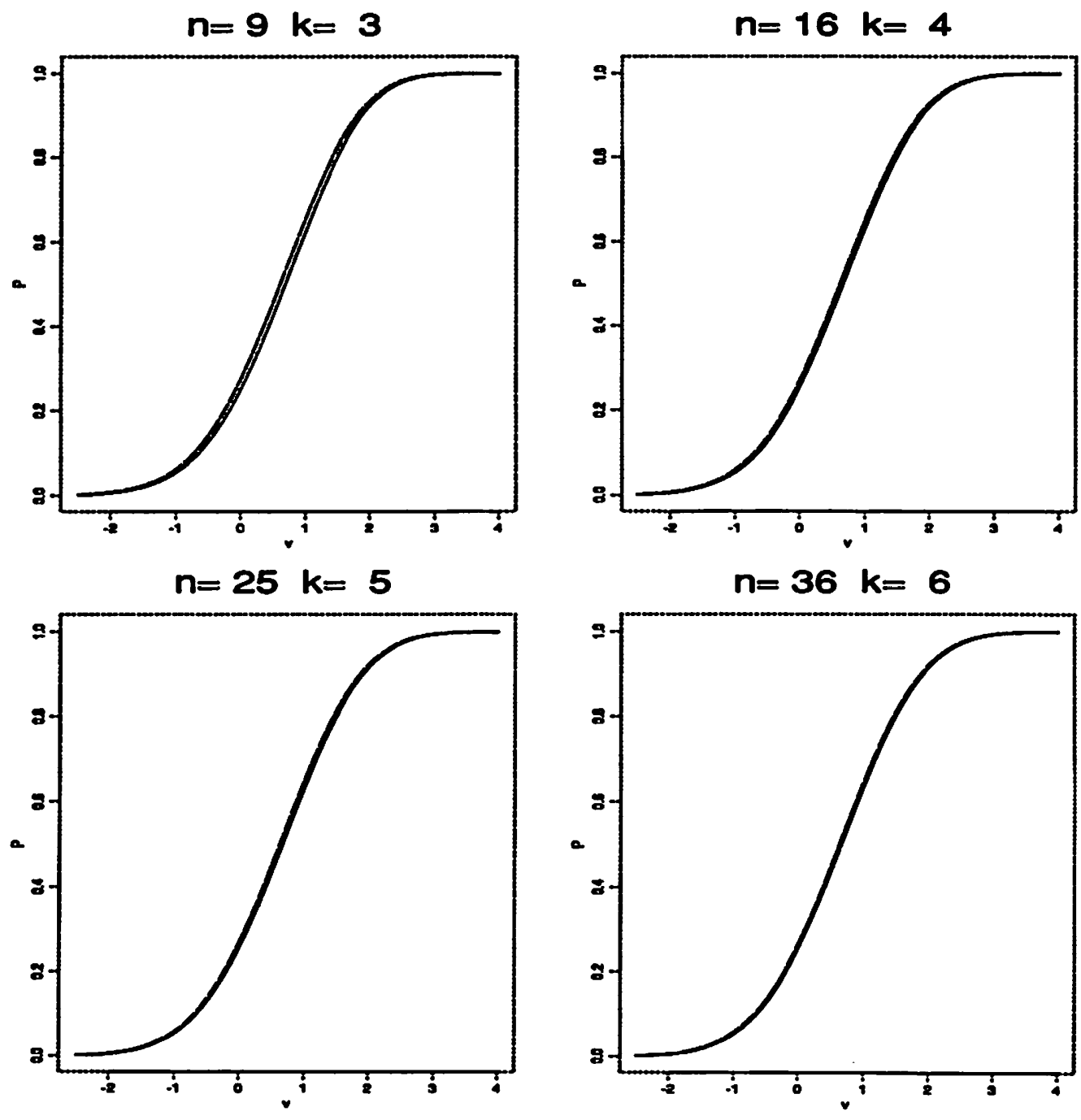

Figure 10: The cumulative distribution of $V_{\alpha}$ when $\hat{z}_{t}=4$ True -, Exact LR - - , Approx LR - - - . 
of $V_{\alpha}=\alpha\left(I^{\alpha \alpha}\right)^{-\frac{1}{2}}$ is given by (2.1) with constants:

$$
\begin{aligned}
& d_{1}=1 /(2 \sqrt{m}) \frac{E\left[\hat{p}^{2}\right]}{1+E\left[\hat{p}^{2}\right]} \\
& d_{2}=-1 /(2 m)\left[\frac{E\left[\hat{p}^{2}\right]}{1+E\left[\hat{p}^{2}\right]}\left(1-\frac{E\left[\hat{p}^{2}\right]}{1+E\left[\hat{p}^{2}\right]}\right)+\frac{2}{1+E\left[\hat{p}^{2}\right]}\left(2-\frac{E\left[(1+\hat{p})^{3}\right]}{E\left[(1+\hat{p})^{2}\right]}\right)\right], \\
& F_{3}=1 / \sqrt{m}, \quad F_{4}=(1 / m)\left(\frac{12}{1+E\left[\hat{p}^{2}\right]}-1\right) .
\end{aligned}
$$

From above, the coefficients of the $O\left(m^{-\frac{1}{2}}\right)$ term in $d_{1}, F_{3}$ are bounded and positive, as is the first term in brackets. The profile likelihood of $\alpha$ has positive skew, and, whether its tails are fatter than normal or not, it can always be fit by the $\log$ of an $F$-distribution, since $F_{3}^{2}+F_{4}>0$. The Laplace approximation will always shift the profile likelihood to the right, but the change will tend to be small. It can either flatten the profile or make it more concentrated, though the latter course of action seems counterintuitive. For large $n, E[\hat{z}]$ should be close to $\mathbf{. 7 8 5}$, so that the quantile orthogonal to the scale parameter should be close to the third quartile. Therefore, the behaviour we described above should be typical of quantiles in that region.

\section{An application : Pike's carcinogenesis data}

Since our approximations for Gumbel errors are data dependent, we will focus on a particular data set for which the Gumbel distribution seems preeminent. Our data comes from a carcinogenicity experiment on mice which has been reported in Pike (1966) and analysed extensively by Kalbfleisch \& Prentice (1980). Since cancer occurs in a tissue when at least one of its cells becomes carcinogenic, Pike argued for a Weibull model for the time till cancer onset, due to the asymptotic derivation of the Weibull as the minimum of a large number of independent observations. The assumptions implicit in the analysis are that (i) death with tumor is equivalent to death from tumor, (ii) the tumor is rapidly lethal and (iii) eventually all mice will contract cancer, if they live long enough. As a result, mice that had not died at the time of the analysis or were tumor-free at death were regarded as censored observations. In Pike's original data set there were two groups of mice, the second containing so many ties as to cast doubt on a continuous Weibull model and argue for an analysis based on interval censoring. The first group is the one used by Geisser (1993) and we shall focus on it alone. It is given in table 1. The observation 188 appears twice in Pike's data set, but its second occurrence was omitted by Geisser. We will also exclude it from our initial analysis, keeping it in reserve for a perturbation analysis later on.

Geisser postulated a Weibull threshold model $\left[a\left(L-l_{0}\right)\right]^{b} \sim \exp (1)$ for the actual lifetimes of the observations. This is equivalent to assuming a Gumbel location-scale model for the shifted loglifetimes $U=\log \left(L-l_{0}\right) \sim \operatorname{Gumbel}\left(\theta_{0}, \exp \left(\theta_{2}\right)\right)$ with $\theta_{0}=-\log a, \theta_{2}=-\log b$. His analysis was performed conditionally on $l_{0}=100, b=3$, where $\hat{l}_{0}=98.9, \hat{b}=3.11$ are 
$143164188190192206209213216220227230234246265304216^{+} 244^{+}$

Table 1: Days to vaginal cancer mortality in mice after carcinogenic insult. Data from Pike (1966). Crosses denote censored observations.

\begin{tabular}{lrrrrrrrcc}
\hline Full & 14 & 37 & 67 & 70 & 72 & 83 & 85 & 86 & 87 \\
& 87 & 86 & 85 & 82 & 72 & 49 & 5 & $5189^{+}$ & $2651^{+}$ \\
& & & & & & & & & \\
Reduced & 22 & 45 & 71 & 73 & 75 & 84 & 85 & 85 & 86 \\
& 85 & 83 & 82 & 80 & 69 & 48 & 10 & $5026^{+}$ & $2610^{+}$ \\
\hline
\end{tabular}

Table 2: Conditional Predictive Ordinates $\times 10^{4}:$ Pike's data.

the MLE's of the extended data set in Pike. We will also condition on $l_{0}=100$, but allow $b$ to be unrestricted, turning the problem into a bivariate one. This way we can compare our approximations to the exact results via at most two-dimensional numerical integrations, using an adaptive quadrature algorithm from Burden \& Faires (1985).

It is not clear whether the extended model is actually to be preferred. Since the prior on $\theta$ is improper, a Bayes factor cannot be calculated. Also, though the reduced model would be chosen by the penalised profile likelihood criteria of both Schwarz (1978) and Akaike (1973), they are based on asymptotic considerations that may not be applicable for a sample size of 18. An alternative method of model selection has been suggested by Gelfand et al. (1992). It is based on calculating the conditional predictive ordinates (CPO) of the observations under both the full and the reduced models, where

$$
C P O_{i}=\int L\left(U_{i} \mid \theta\right) \pi\left(\theta \mid U^{-i}\right) d \theta=\int L\left(U_{i} \mid \theta\right) \frac{\pi\left(\theta \mid U^{-i}\right)}{\pi\left(\theta \mid U^{n}\right)} \pi\left(\theta \mid U^{n}\right) d \theta
$$

In table 4.2 we present simulation consistent estimates of the $C P O$ 'sbased on 50,000 replications from the posterior of the full model. The exact $\log F$ predictive density of $U_{i} \mid U_{-i}$ for the reduced model is available by the results in Geisser (1993). The CPO's for the last two observations are much larger in value because they represent survival probabilities, not density ordinates. Since $\log \prod_{i=1}^{18} C P O_{F i}=-85.00467<-83.81247=\log \prod_{i=1}^{18} C P O_{R i}$, the CPO criterion provides slight evidence in favour of the reduced model.

A plot of the CPO's for each model, scaled by their maximum, is given in fig. 11, with the two censored observations excluded. The two smallest and the largest observed lifetimes appear discordant, though a predictive significance test for the reduced model by Geisser (1989) did not result in labelling observation 16 an outlier. The plot at the bottom of fig. 11 shows the ratio of the CPO's for the 2 models and reveals that the reason the reduced model is preferred is essentially its ability to better predict the three most extreme observed lifetimes, points where the density is very low to start with and where small changes in the 

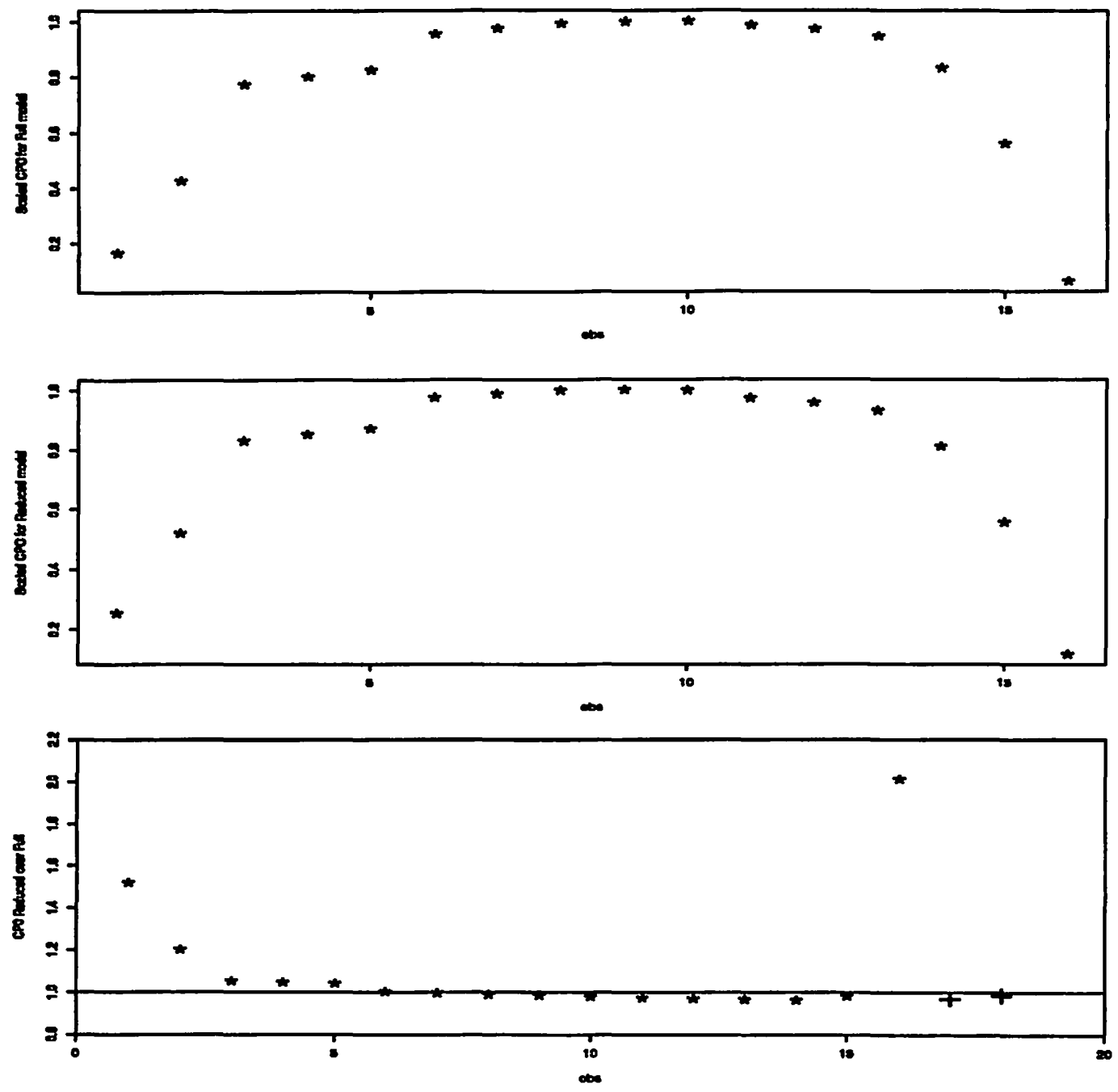

Figure 11: Conditional Predictive Ordinate plots for Pike's data. 
density estimates can result in large differences in the CPO ratio. For the other points the two models are essentially equivalent.

The hypotheses of interest to us were assumed to be expressible in terms of either the survivor function $S_{l}=P\left[U \geq u_{l}\right]=P[U \geq \log (l-100)]$ or the $p^{\prime}$ th quantile $U_{p}$ of the shifted loglifetimes. For Gumbel errors, the latter are both parametric functions of $\theta$ given by:

$$
\begin{aligned}
S_{l}(\theta) & =\exp \left(-\exp \left[\left(Z_{l}(\theta)\right]\right), \text { for } Z_{l}(\theta)=\left(u_{l}-\theta_{0}\right) e^{-\theta_{2}}\right. \\
U_{p}(\theta) & =\theta_{0}+e^{\theta_{2}} \log [-\log (1-p)] .
\end{aligned}
$$

It is easy to show that $P\left[S_{l} \geq 1-p\right]=P\left[Z_{l} \leq \log (-\log [1-p])\right]=P\left[U_{p} \geq u_{l}\right]$, so that hypotheses on the survivor function can be tested by approximating the marginal of either $Z_{l}$ or $U_{p}$. If a sensitivity analysis is entertained, the choice of marginal to be approximated depends on whether one intends vary $p$ or $l$. In what follows we shall assume that we are interested in the marginal posteriors of the median lifetime $L_{.50}$, the probability of survival beyond 210 days $S_{210}$ and of the power parameter $b$ of the Weibull. Those, in turn, are found by transforming the exact posteriors density of $U_{.50}, Z_{210}$ and $\theta_{2}$ respectively.

The posterior mode of $\theta$ under a flat prior is given by $\left(\hat{\theta}_{0}, \hat{\theta}_{2}\right)=(4.8953,-1.2267)$. Since the median of the standard Gumbel distribution is -.3665 , we let

$$
\alpha=\left(\theta_{0}-\hat{\theta}_{0}\right) \exp \left(-\hat{\theta}_{2}\right)-0.3665\left(\exp \left(\theta_{2}-\hat{\theta}_{2}\right)-1\right), \delta=\left(\theta_{2}-\hat{\theta}_{2}\right)
$$

be a data-dependent reparameterisation that shifts the posterior mode to the origin. The inverse of observed information matrix of $\omega=(\alpha, \delta)$ evaluates at $\hat{\omega}=0$ to

$$
I^{-1}(\hat{\omega})=\left(\begin{array}{ll}
I^{\alpha \alpha} & I^{\alpha \delta} \\
I^{\alpha \delta} & I^{\delta \delta}
\end{array}\right)=\left(\begin{array}{rr}
0.0786 & -0.0240 \\
-0.0240 & 0.0358
\end{array}\right)
$$

and can be used to translate the asymptotic approximations in terms of the standardised variable $V$ back to the original $\theta$ scale.

The Laplace approximation to the posterior of $V_{\delta}=\delta\left(I^{\delta \delta}\right)^{-\frac{1}{2}}$ can be expanded as in (2.1) with constants $d_{1}=0.1892, d_{2}=0, F_{3}=0.3310, F_{4}=-0.1048$, so that $\left(\theta_{2}+1.1897\right) / 0.5484 \stackrel{d}{\approx} \log F(827.5242,16.0528)$. Setting $d_{1}=d_{2}=0$ gives us an approximation to the profile posterior. We find that $\left(\theta_{2}+1.2267\right) / 0.5484 \stackrel{d}{\approx} \log F(826.4275,17.1496)$ in the original $\theta_{2}$ scale. As suggested by the sign of $d_{1}$, the determinant term shifts the profile mode to the right, from -1.2267 to -1.1897 , and increases its right skewness by reducing the denominator degrees of freedom. Due to its linearity $\left(d_{2}=0\right)$ it leaves the scale unaffected at $\mathbf{0 . 5 4 8 4}$. In both cases, the numerator degrees of freedom of the $F$ distribution are so large that little accuracy is lost by taking it to be a scaled inverse $\chi^{2}$. Compared to the normal, both the Laplace and the profile posterior have a longer right tail $\left(F_{3}>0\right)$. In fig. 12 we superimpose the profile and Laplace posteriors on the exact marginal of $\theta_{2}$ and find that both Laplace posteriors are indistinguishable from the exact density to within plotting accuracy. 


\begin{tabular}{|l|cc|cc|}
\hline & \multicolumn{2}{|c|}{ QUANTILES } & \multicolumn{2}{c|}{ MOMENTS } \\
\hline METHOD & $\mathbf{0 . 0 2 5}$ & $\mathbf{0 . 9 7 5}$ & Mean & S. D. \\
\hline Exact & -1.5193 & $-\mathbf{0 . 7 3 3 4}$ & -1.1562 & $\mathbf{0 . 2 0 0 1}$ \\
Laplace App. & -1.5192 & $-\mathbf{0 . 7 3 2 0}$ & -1.1595 & $\mathbf{0 . 2 0 0 5}$ \\
Laplace logF & -1.5173 & -0.7276 & -1.1555 & $\mathbf{0 . 2 0 1 6}$ \\
Profile App. & -1.5459 & -0.7831 & -1.1954 & 0.1945 \\
Profile logF & -1.5455 & -0.7825 & -1.1947 & 0.1947 \\
Normal & -1.5975 & -0.8558 & -1.2267 & 0.1892 \\
\hline
\end{tabular}

Table 3: Posterior quantiles and moments of the logscale parameter $\theta_{2}$.

To calculate a $95 \%$ equal-tail credible interval for $\theta_{2}$ and its first two moments we used: (i) numerical integration of the exact posterior, (ii) asymptotic expansion of both the Laplace and the profile posterior, (iii) the $\log F$ approximation to the Laplace and profile posteriors given above and, finally, (iv) a normal approximation, centered at the $\theta_{2}$ component of the joint posterior mode and using the observed information at that point. The results in table 3 show the superiority of the Laplace approximation, with its asymptotic expansion enjoying two-digit accuracy.

In fig. 13 we return to a scale more meaningful to us and plot the exact posterior of the power of the Weibull $b=\exp \left(-\theta_{2}\right)$. Its mean and standard deviation can be found by (2.2), since $E\left[b^{k}\right]=E\left[\exp \left(-k \theta_{2}\right)\right]=M_{V_{6}}\left(-k \sqrt{I^{\delta \delta}}\right) \exp \left(-k \hat{\theta}_{2}\right)$. They are given by $(3.252,0.6401)$ and compare well with the numerical integration results $(3.2406,0.6342)$. Transforming the $95 \%$ credible intervals given for $\theta_{2}$ above, we obtain a range of possible power transformations of Pike's data to exponentiality given by $(2.08,4.57)$. This implies that $b$ is a posteriori almost certainly greater that one, i.e. the hazard increases with time. The significant spread of the posterior of $b$ around its modal value also suggests that setting $b$ equal to 3 will lead to credible intervals for the other parameters that are too short, unless, of course, this happens to be the true value.

The Laplace approximation to the posterior of $V_{\alpha}=\alpha\left(I^{\alpha \alpha}\right)^{-\frac{1}{2}}$ can be expanded as in eqn. (2.1) with constants $d_{1}=0.0838, d_{2}=0.0362, F_{3}=-0.2560, F_{4}=0.2290$, which imply that $\left(U_{.50}-4.7949\right) / 0.0992 \stackrel{d}{\approx} \log F(4.4532,8.0585)$. The profile posterior alone is well approximated by $(U .50-4.7879) / 0.1016 \stackrel{d}{\approx} \log F(4.6428,8.9402)$. The magnitude of the linear and quadratic terms of the determinant expansion indicates that the Laplace posterior differs little from the profile, shifting its mode slightly to the left $\left(d_{1}>0\right)$ and flattening it a bit $\left(d_{2}>0\right)$. Here the scale change largely manifests itself as a slight loss of degrees of freedom. More significant is the difference with the normal approximation, which has too short a left tail $\left(F_{3}<0\right)$ and is too tight $\left(F_{4}>0\right)$.

In fig. 14 we plot the exact, Laplace and profile posteriors. Though barely discernible from the plot, the Laplace approximation differs slightly from the exact marginal in the 


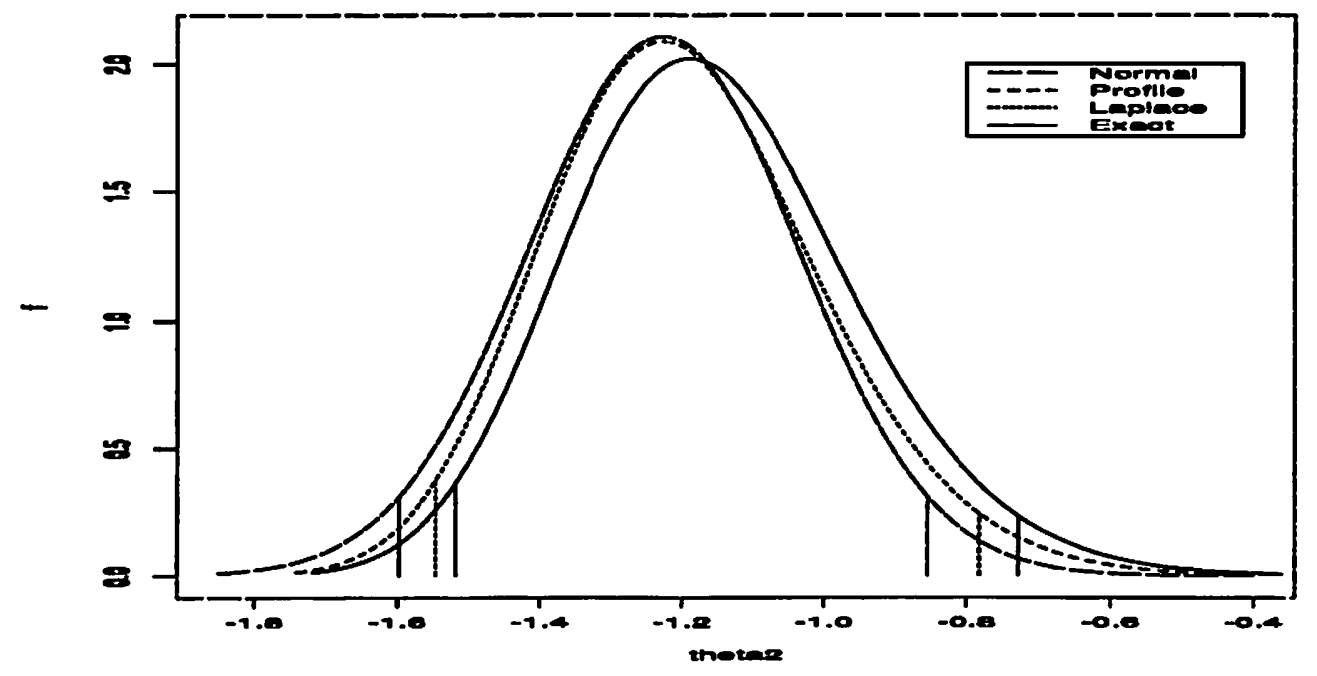

Figure 12: The marginal posterior of the logscale parameter $\theta_{2}$

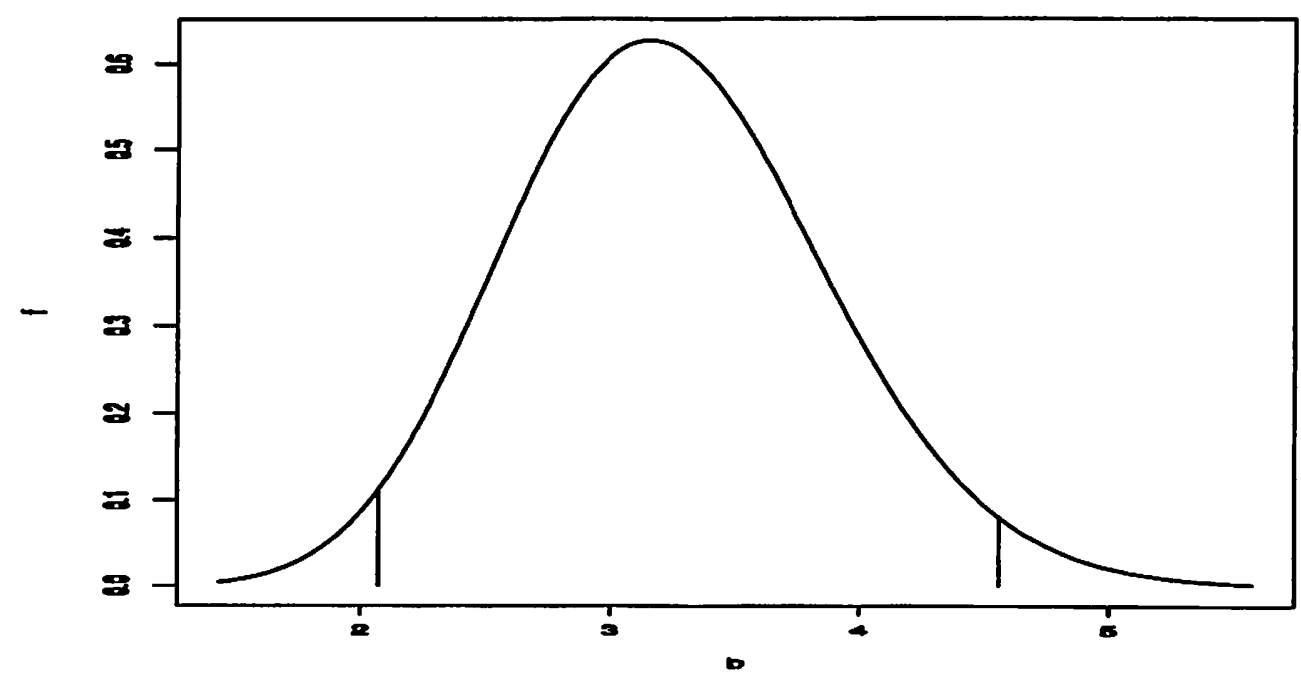

Figure 13: The marginal posterior of the power parameter $b$ 


\begin{tabular}{|l|cc|cc|}
\hline & \multicolumn{2}{|c|}{ QUANTILES } & \multicolumn{2}{c|}{ MOMENTS } \\
\hline METHOD & 0.025 & 0.975 & Mean & S. D. \\
\hline Exact & 4.5927 & 4.9528 & 4.7836 & 0.1052 \\
Laplace App. & 4.5932 & 4.9524 & 4.7842 & 0.0902 \\
Laplace logF & 4.5916 & 4.9528 & 4.7837 & 0.0912 \\
Profile App. & 4.5868 & 4.9433 & 4.7773 & 0.0893 \\
Profile logF & 4.5858 & 4.9424 & 4.7762 & 0.0902 \\
Normal & 4.6267 & 4.9490 & 4.7878 & 0.0822 \\
\hline
\end{tabular}

Table 4: Posterior quantiles and moments of the median loglifetime $U_{.50}$.

\begin{tabular}{|l|cc|cc|}
\hline & \multicolumn{2}{|c|}{ QUANTILES } & \multicolumn{2}{c|}{ MOMENTS } \\
\hline METHOD & 0.025 & 0.975 & Mean & S. D. \\
\hline Exact & -1.3109 & -0.0965 & -0.6618 & 0.3094 \\
Laplace App. & -1.3076 & -0.0940 & -0.6614 & 0.3098 \\
Profile App. & -1.3211 & -0.1012 & -0.6712 & 0.3113 \\
Normal & -1.2304 & -0.0292 & -0.6298 & 0.3065 \\
\hline
\end{tabular}

Table 5: Posterior quantiles and moments of the standardised residual at 210 days

extreme tails. Calculating $95 \%$ equal-tail credible intervals for the median loglifetime $U_{.50}$, we find that the Laplace approximation enjoys two-digit accuracy in both its asymptotic expansion and $\log F$ approximations, with the profile posterior trailing not far behind. Table 4 also shows that the Laplace approximations tend to underestimate the posterior variance, with the normal approximation performing even worse. In fig. 15 we move to a more natural scale and show the interim posterior distribution of the median lifetime $L_{.50}$. It is seen to have negligible mass beyond $(180,260)$ days, with $95 \%$ interval endpoints given by $(198.8,241.4)$.

Finally, we focus on the marginal of $Z_{210}$. Due to nonconstancy of the Jacobian, the joint posterior of $\left(Z_{210}, \theta_{2}\right)$ is now maximised at $(-.6298,-1.1897)$, instead of $(-0.6645,-1.2267)$. Since $I^{z z}=0.0939, d_{1}=0.0322, d_{2}=-0.0014, F_{3}=-0.2703, F_{4}=-0.0820$, a $\log F$ approximation is not available in this case for either the Laplace or the profile posterior of $Z_{210}-\hat{Z}_{210}$. The two approximations are close $\left(d_{1}, d_{2} \approx 0\right)$, but differ from the normal in that they transfer weight from the right to the left tail. The remarkable accuracy of the Laplace approximation is evidenced from the results reported in table 5 and from fig. 17, where it cannot be told apart from the exact density to within plotting accuracy. The $95 \%$ credible intervals for $Z_{210}$ tranform into a range for the survival probability beyond 210 days of $(.40, .76)$. The exact posterior of the latter is given in fig. 17 . 


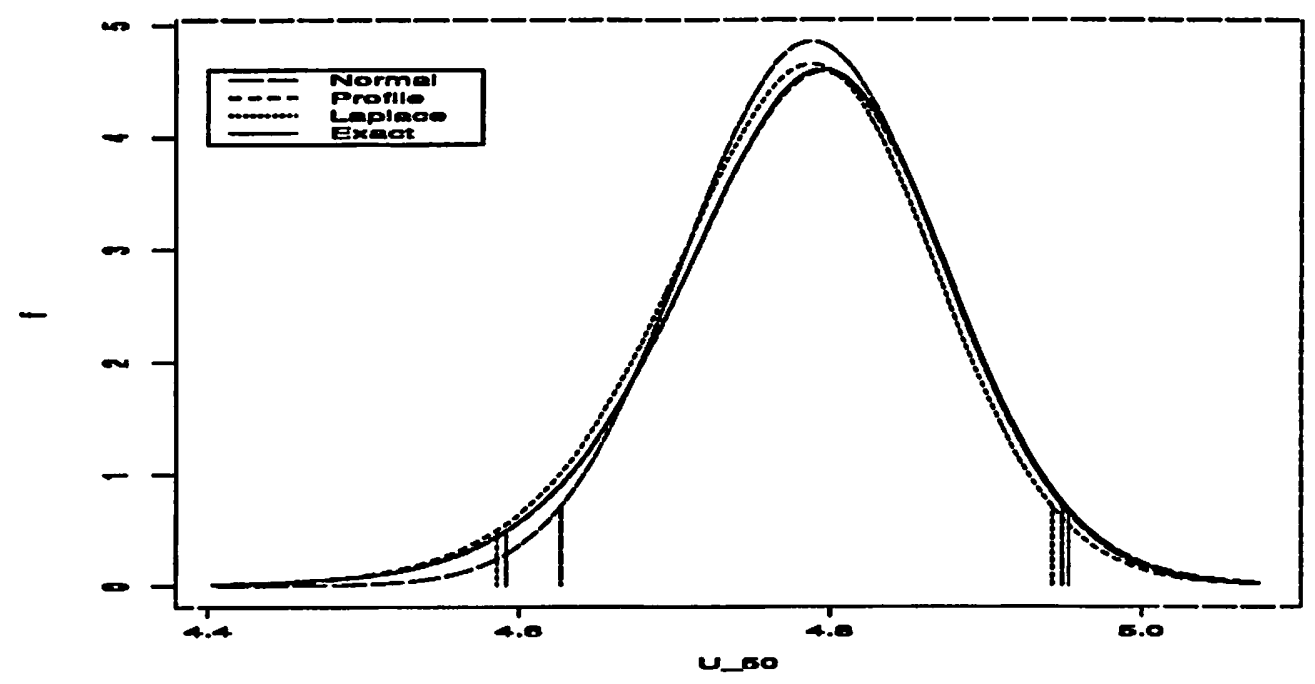

Figure 14: The marginal posterior of the median loglifetime $U_{.50}$

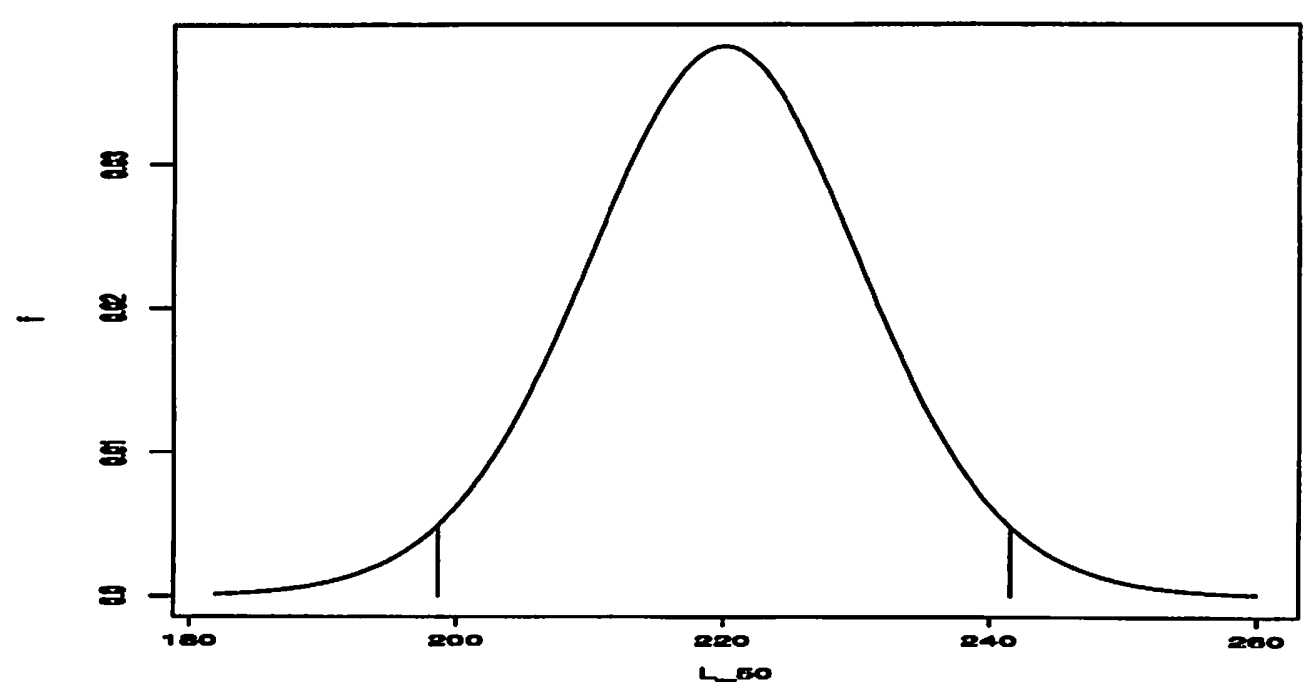

Figure 15: The marginal posterior of the median lifetime $L_{.50}$ 


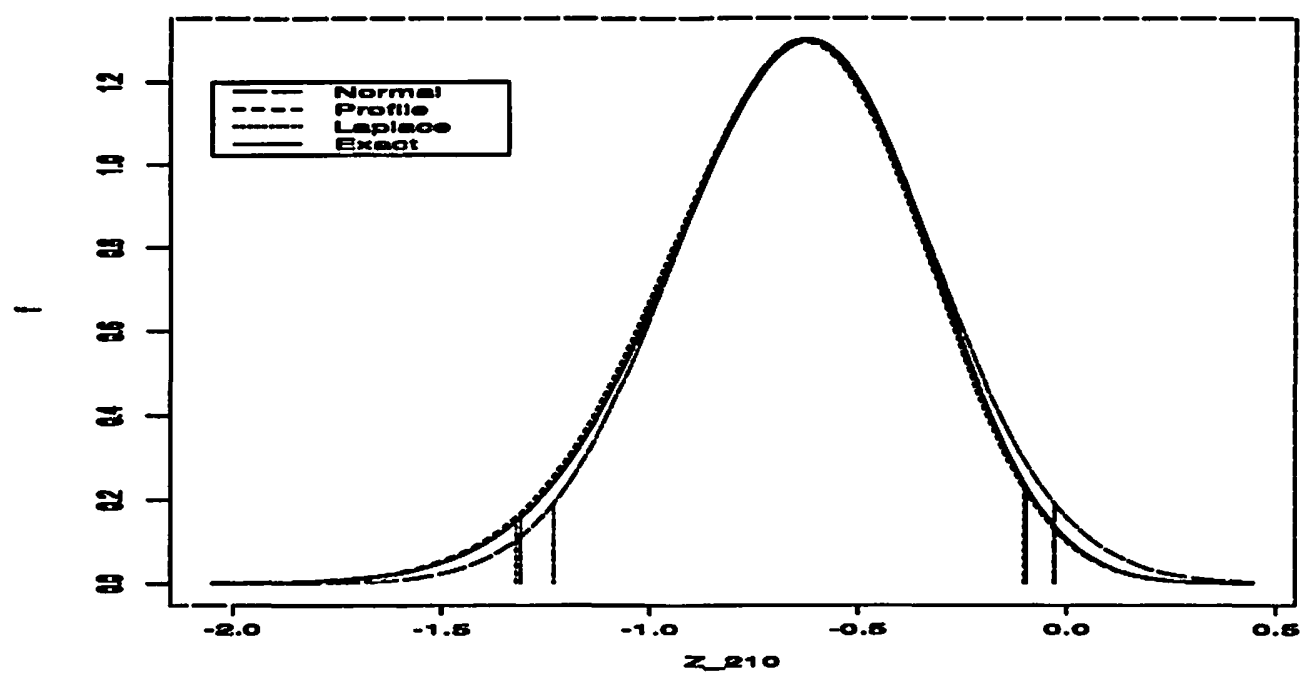

Figure 16: The marginal posterior of the standardised residual at 210 days

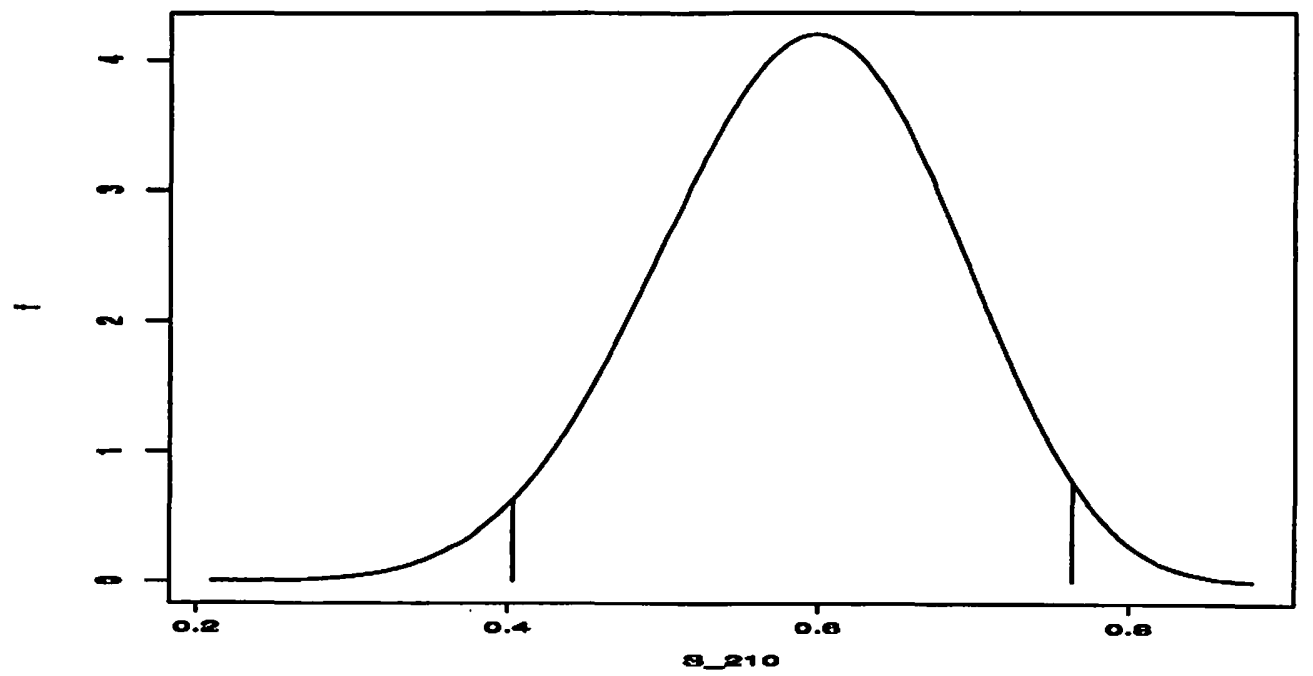

Figure 17: The marginal posterior of the probability of survival beyond 210 days 


\subsubsection{Sensitivity Analysis}

The machinery we have developed makes it fairly easy for us to examine the effect on lower dimensional margins of $O(1)$ perturbations of the joint posterior of $\omega$. In regression models where the error density is strictly positive and bounded above, such as the loggeneralised gamma family, single case deletion/addition falls in this category.

The loglikelihood contribution of the $i$ 'th case to the joint likelihoods $L(\omega)$ defined in (3.6), (3.21) is given by: $L_{i}(\omega)=\delta\left(I_{c_{i}}-1\right)-g\left[z_{i}(\omega)\right]$ where $I_{c_{i}}$ is a censoring indicator. Deletion of the $i$ 'th case affects two terms of the Laplace approximation (2.12): the profile likelihood $R\left(\omega_{1}\right)$ and the determinant term $h\left(\omega_{1}\right)$. Standard results on determinants allow us to express the change in $h\left(\omega_{1}\right)$ as $O\left(k n^{-\frac{3}{2}}\right)$. This can be neglected to second order under the assumption of a fixed number of parameters and an ever increasing number of observations. In actual practice neglecting this term incurs a penalty that increases with dimensionality. The corresponding change to $R\left(\omega_{1}\right)$ is given by

$$
R_{i}\left(\omega_{1}\right)=L_{i}\left(\hat{\omega}\left(\omega_{1}\right)\right)-L_{i}(\hat{\omega})=\hat{\delta}\left(\omega_{1}\right)\left(I_{c_{i}}-1\right)+g\left[z_{i}(\hat{\omega})\right]-g\left[z_{i}\left(\hat{\omega}\left(\omega_{1}\right)\right)\right] \text {. }
$$

Rather than finding the new profile mode and recalculating the expansion of $R\left(\omega_{1}\right)$, it is more efficient to absorb the change in the prior term $w\left(\omega_{1}\right)$ of $(2.12)$ and leave $R\left(\omega_{1}\right)$ as is. A quadratic expansion of the new $w\left(\omega_{1}\right)$ around the original profile likelihood mode changes the constants $d_{1}, d_{2}$ of (2.1), leaving $F_{3}, F_{4}$ as before. It can be shown that all quantities involved are byproducts of the marginal expansions around the original profile mode and, hence, readily available from earlier stages of the analysis.

We illustrate simple likelihood perturbations using Pike's data set. The effect of deleting individual cases on the logscale parameter, the median loglifetime and the residual at 210 days can be assessed and compared across variables by finding the mean, median and $95 \%$ equal tail credible intervals for $V$, when the parameter of interest $\omega_{1}$ is successively set equal to $\theta_{2}-\hat{\theta}_{2},\left(U_{.50}-\hat{U}_{.50}\right) \exp \left(\hat{\theta}_{2}\right)$ and $Z_{210}-\hat{Z}_{210}$.

Using the above, we first plot in fig. 18 the mean of $V$ vs. the number of the deleted observation. Since the mean of $V$ is equivalent to the distance in standard units between the mean and the mode of the unstandardised version of the variable, the quantity being plotted can also be thought of as measuring Pearson skewness in the original scale. In fig. 19 we go further and show how single case deletion affects the $.025, .500$ and .975 quantiles of the posterior of $V$. Dotted horizontal lines at $-1.96,0$ and 1.96 serve as benchmarks for the departure from normal shape.

The plots shows that the positive skew of the posterior of $\theta_{2}$ is mostly due to the three most extreme observed lifetimes, observations $1,2,16$. Their omission brings the posterior much closer to normality. None of the other fifteen observations seems terribly influential. The skewness in the posterior of $U_{.50}$ changes gradually from positive to negative as larger lifetimes are ommitted, a quite intuitive result. Here, the two smallest observed lifetimes balance out the largest, with the deletion of any one of them exacerbating the skewness of the posterior. Finally, removal of the two smallest observations results in the density of 

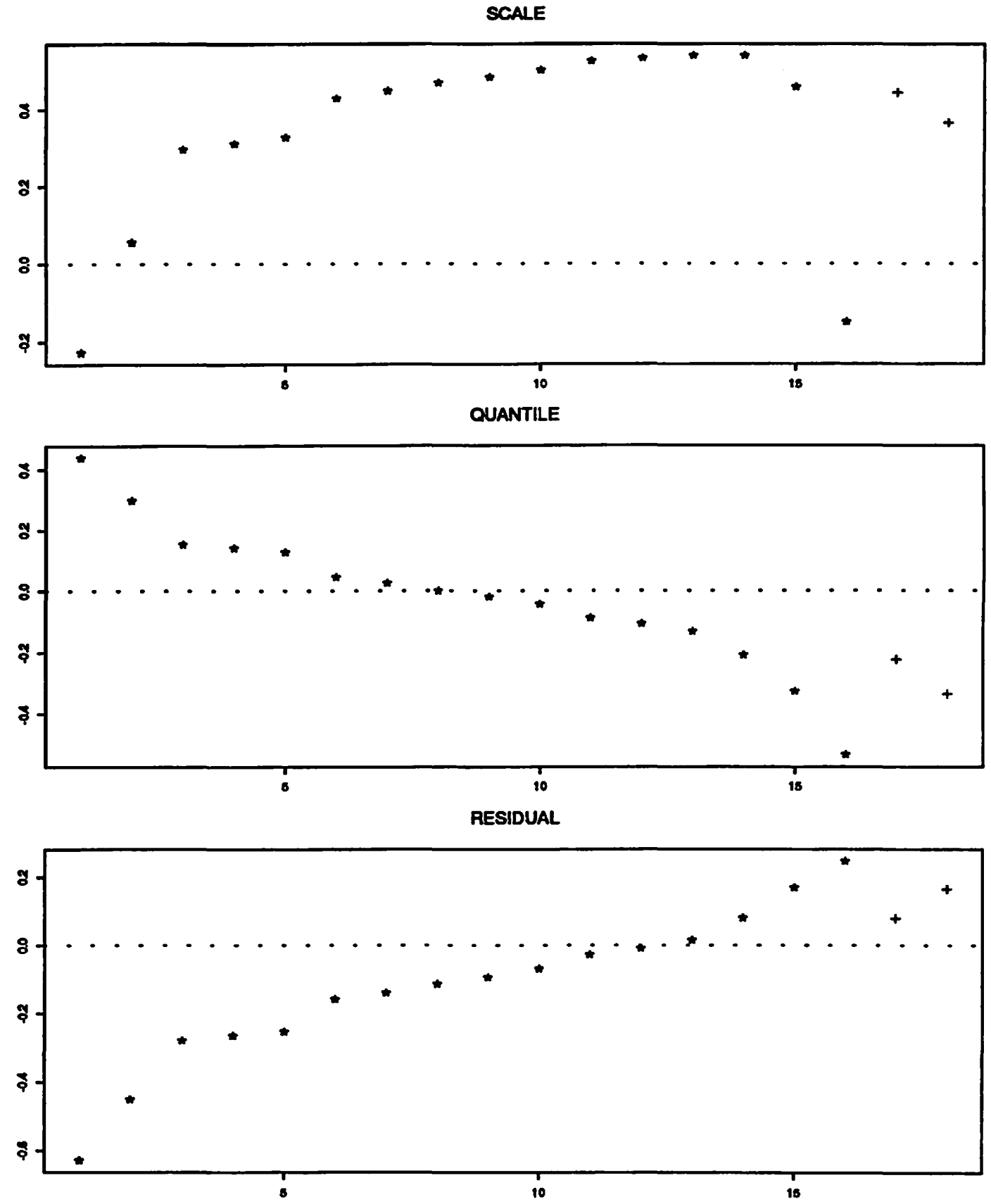

Figure 18: Approximate Pearson skewness vs. number of observation deleted.

Crosses denote censored observations. 

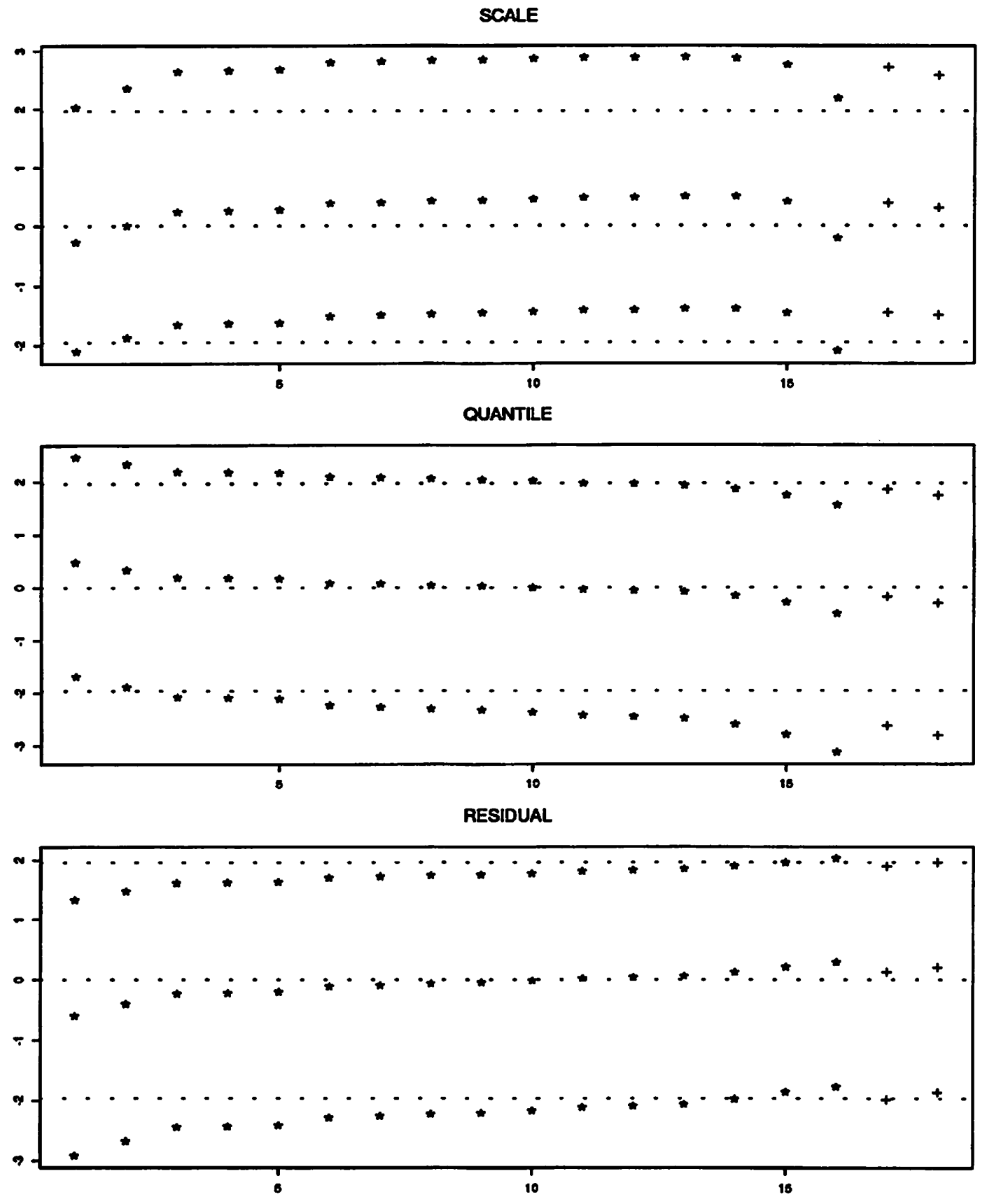

Figure 19: Medians and 95\% equal tail intervals for the standardised versions of the variables of interest vs. number of observation deleted. Crosses denote censored observations. 
$Z_{210}$ acquiring a large left tail, but it remains close to normal when the largest lifetime is deleted.

It should be noted that case 16 , which appeared heavily influential in the posterior of $\theta_{2}$, is much less $s 0$ in that of $Z_{210}$. Any summary measure of case influence would fail to unmask this difference, whereas we are able to focus directly on the quantities of interest and assess influence on each marginal posterior separately, with a negligible increase in computational burden. If select tail probabilities or other low order moments are of interest, they can also be easily calculated.

Since the approximations above are based on an expansion around the original mode of $L(\omega)$, they should be less accurate than the ones that involve changing $L(\omega)$ itself and remaximising. To assess the extent of the anticipated loss of accuracy, Papandonatos (1994) took into account the extra observation at 188 days that was omitted from our earlier analysis and compared both the direct and indirect expansions with the exact results. The two-digit accuracy of the approximations to credible intervals and moments was maintained in all cases, suggesting that, in a location-scale model, the penalty we pay for using the indirect instead of the direct expansion seems small compared to the substantial savings we realise in terms of computation time. Observation 3 is quite typical of the mass of the data and similar results should obtain for all the other lifetimes, but cases 1,2 and 16. Doubling the weight of the most extreme lifetimes should cause a much larger shift in the posterior mode and increase the discrepancy between the 2 approximations.

\section{Conclusion}

Extensive checking of our results has indicated that their accuracy depends critically on the dimensionality of the regression model, less so on its degrees of freedom. This limitation follows directly from our assumption of a fixed number of regressors and a sample size that grows without bounds. Since in the end we have to analyse data sets where both are fixed, the second order correctness of our results can be misleading as to their accuracy, if the ratio of regressors to the sample size is large. As a rule of .. thumb, we would like the number of regressors not to exceed the square root of the sample size, but expect our approximations to be adequate for location-scale models even for sample sizes as low as 15 .

As mentioned earlier, a judicious reparameterisation of the model may increase the accuracy of our approximation to the marginals of interest. The emphasis should be on finding a parameterisation in which the information about the nuisance parameters changes slowly with the parameter of interest, when evaluated at the constrained likelihood mode. Before going through the time-consuming process of coding the derivative arrays required for our expansions. one may first want to visually compare the determinant term of (2.12) for various parameterisations of choice, using the purely numerical approximation implemented by Tierney (1990) in LISP-STAT. To get satisfactory results one should also be prepared to look beyond pivotal quantities, thus sacrificing the connection with conditional frequen- 
tist inference and adopting a purely Bayesian approach. Comparison of (3.10)-(3.13) with (3.23)-(3.26) shows that the intuition gained in simplifying the expansions of one parameterisation may well carry over to another setting.

Conspicuous in their absence from this work have been any simplifications involving the prior. The reason is twofold: (i) we intended to focus on a reference analysis based on a flat prior and (ii) no conjugate prior usually exists for censored linear regression models, so that each prior must be treated in an ad hoc manner and there is little scope for obtaining for it the kind of simplifications we achieved for the likelihood. On the other hand, only the first two derivatives of the prior need to be computed, a task much less arduous than finding the third and fourth derivative arrays of the likelihood.

One rather surprising aspect of the results presented herein has been the good performance of the $\log F$ approximation to both low order moments and equal tail intervals of the posteriors examined in section 4. Since the use of a parametric family of distributions can considerably simplify our calculations, it is worth considering whether this observation has wider applicability or is limited to the particular data set examined here. The papers by Sweeting (1987, 1988), Sprott \& Viveros (1987) and our own experience with other Gumbel and censored Normal data sets from the literature suggest that the excellence of the approximation to the posteriors of the log scale parameter and of central quantiles of the response is not fortuitous, but can be widely expected within the generalised log-Gamma family of distributions. On the other hand, extreme quantiles and survival probabilities usually fail to admit a $\log F$ approximation because of the large negative magnitude of the $F_{4}$ constant in the asymptotic expansion (2.1). When a $\log F$ density can be fit, it tends to provide good approximations to both low order moments and equal tail intervals. Though its unavailability does not necessarily imply inadequacy of the Laplace posterior per se, its existence can be a boon in situations such as prediction, where a certain density is to be estimated by a weighted sum of Laplace posteriors; normalising each one of them individually can be quite cumbersome and time-consuming. An alternative to numerical integration, in this case, is calculation of the density from formula (2.6), using finite differences, but this method is unreliable in general, since it exhibits a disconserting tendency to turn negative in the extreme tails.

\section{References}

Akaike, H. (1973). Information theory and an extension of the maximum likelihood principle. Proceedings of the 2nd International Symposium on Information Theory (eds B.N. Petrov, and F. Czaki), pp. 267-281. Tokyo: Akademiai Kiado.

Barndorff-Nielsen, O.E. (1983). On a formula for the distribution of the maximum likelihood estimator. Biometrika, 70, 343-365.

Chaloner, K. \& Brant, R. (1988). A Bayesian approach to outlier detection and residual analysis. Biometrika, 75, 651-659. 
Chen, C-F. (1985). On asymptotic normality of limiting density functions with Bayesian implications. J. Royal Statist. Soc. B. 47, 540-546.

Cox, D.R. \& Reid, N. (1987). Parameter orthogonality and approximate conditional inference. J. Royal Statist. Soc. B. 49, 1-39.

DiCiccio, T.J. (1987). Approximate inference for the generalised gamma distribution. Technometrics, 29, 33-40.

DiCiccio, T.J., C.A., Field, C.A. \& Fraser, D.A.S. (1990). Approximations of marginal tail probabilities and inference for scalar parameters. Biometrika, 77, 77-95.

DiCiccio, T.J. \& Field, C.A. (1991). An accurate method for approximate conditional and Bayesian inference about linear regression models from censored data. Biometrika, 78, 903-910.

Efron, B. \& Hinkley, D.V. (1978). Assessing the accuracy of the maximum likelihood estimator: Observed versus expected Fisher information. Biometrika, 65, 457-487.

Fraser, D.A.S. \& Reid, N. (1993). Third order asymptotic models: Likelihood functions leading to accurate approximations for distribution functions. Statistica Sinica, 3, 67-82.

Gelfand, A.E., Dey, D.K. \& Chang, H. (1992). Model determination using predictive distributions with implementation via sampling-based methods. Bayesian Statistics 4 (eds J.M. Bernardo, J.O. Berger, A.P. Dawid and A.F.M. Smith), pp. 147-167. Oxford University Press.

Geisser, S. (1989). Predictive discordancy tests for exponential observations. The Canadian Journal of Statistics, 17, 19-26.

Geisser, S. (1993). Bayesian interim analysis of censored exponential observations. Statistics \& Probability Letters, 18, 163-168.

Hinkley, D.V. (1978). Likelihood inference about location and scale parameters. Biometrika, 65, 253-261.

Hodges, J.S. (1987). Assessing the accuracy of normal approximations. J. Amer. Statist. Assoc., 82, 149-154.

Johnson, N.L. \& Kotz, S. (1970). Continuous Univariate distributions-2. Houghton Mifflin. Johnson, R.A. (1970). An asymptotic expansion for posterior distributions. Annals of Mathematical Statistics, 38, 1899-1907.

Kalbfleisch, J.D. \& Prentice, R.L. (1980). The statistical analysis of failure time data. New York: John Wiley \& Sons.

Kass, R.E. (1988). Comment on 'Saddlepoint Methods' by N. Reid. Statistical Science, 3, 234-236.

Kass, R.E. \& Steffey, D. (1989). Approximate Bayesian inference in conditionally independent hierarchical models. J. Amer. Statist. Assoc., 84, 717-726.

Kass, R.E. \& Slate, E.H. (1992). Parameterisation and diagnostics of posterior nonnormality. Bayesian Statistics 4 (eds J.M. Bernardo, J.O. Berger, A.P. Dawid and A.F.M. Smith), pp. 289-305. Oxford University Press.

Kass, R.E., Tierney, L. \& Kadane, J.B. (1990). The validity of posterior expansions based on Laplace's method. Bayesian and likelihood methods in Statistics and Econometrics 
(eds S. Geisser, J.S. Hodges, S.J. Press and A. Zellner), pp. 472-488. Elsevier Science Publishers B.V.

Leonard, T. (1982). Comment on 'A simple predictive density function' by Lejeune \& Faulkenberry. J. Amer. Statist. Assoc., 77, 657-658.

Lawless, J.F. (1982). Statistical models and methods for lifetime data. John Wiley \& Sons. Lindley, D.V. (1980). Approximate Bayesian Methods. Bayesian Statistics 1 (eds J.M. Bernardo, M.H. DeGRoot, D.V. Lindley and A.F.M. Smith), pp. 223-245, Valencia, Spain: University Press.

Nicolaou, A. (1993). Bayesian Intervals with good frequentist behaviour in the presence of nuisance parameters. J. Royal Statist. Soc. B., 55, 377-390.

Papandonatos, G.D. (1994). Interim analysis of regression models with possible censoring. Unpublished PhD. Dissertation, University of Minnesota.

Peers, H.W. (1965). On confidence points and Bayesian probability points in the case of several parameters. J. Royal Statist. Soc. B., 27, 9-16.

Pike, M.C. (1966). A method of analysis of a certain class of experiments in carcinogenesis. Biometrics, 22, 142-161.

Schwarz, G. (1978). Estimating the dimension of a model. Annals of Statistics, 6, 461-464. Severini, T.A. (1993). Bayesian intervals which are also confidence intervals. J. Royal Statist. Soc. B., 55, 533-540.

Sprott, D.A. (1980). Maximum likelihood: Estimation in the presence of nuisance parameters. Biometrika, 67, 515-523.

Stein, C.M. (1985). On the coverage probability of confidence sets based on a prior distribution. Sequential Methods in Statistics, 16, 485-514. Warsaw: PWN-Polish Scientific Publishers.

Sweeting, T.J. (1987). Approximate Bayesian Analysis of censored survival data. Biometrika, 74, 809-816.

Sweeting, T.J. (1987). Comment on 'Parameter orthogonality and approximate conditional inference' by D.R. Cox and N. Reid. J. Royal Statist. Soc. B., 49, 20-21.

Sweeting, T.J. (1988). Approximate posterior distributions in censored regression models. Bayesian Statistics 3 (eds J.M. Bernardo, M.H. DeGRoot, D.V. Lindley and A.F.M. Smith), pp. 791-799. Oxford University Press.

Tierney, L. (1990). LISP-STAT: An object-oriented environment for statistical computing and dynamic graphics. John Wiley \& Sons.

Tierney, L. and Kadane, J. B. (1986). Accurate approximations for posterior moments and marginal densities. J. Amer. Statist. Assoc., 81, 82-86.

Tierney, L., Kass, R. E. \& Kadane, J. B. (1989). Fully exponential Laplace Approximations to expectations and variances of nonpositive functions. J. Amer. Statist. Assoc., 84, 710716.

Viveros, R. \& Sprott, D.A. (1987). Allowance for skewness in maximum likelihood estimation with application to the location-scale model. Canadian Journal of Statistics, 15, 349-361. 
Welch, B.L. \& Peers, H.W. (1965). On formulae for confidence points based on intervals of weighted likelihoods. J. Royal Statist. Soc. B., 25, 318-329.

Zellner, A. (1975). Bayesian analysis of regression error terms. J. Amer. Statist. Assoc., 70, 138-144. 